\title{
The Search for Heavy Majorana Neutrinos
}

\author{
Anupama Atre ${ }^{1,2}$, Tao Han Ha, $^{2,3}$, Silvia Pascoli ${ }^{5}$, Bin Zhang ${ }^{4 *}$ \\ ${ }^{1}$ Fermi National Accelerator Laboratory, MS106, P.O.Box 500, IL 60510, U.S.A. \\ ${ }^{2}$ Kavli Institute of Theoretical Physics, University of California, Santa Barbara, CA \\ 9310\%, U.S.A. \\ ${ }^{3}$ Department of Physics, University of Wisconsin, 1150 University Ave, Madison, WI \\ 53706, U.S.A. \\ ${ }^{4}$ Center for High Energy Physics, Department of Physics, Tsinghua University, Beijing \\ 100084, P.R. China \\ ${ }^{5}$ Institute for Particle Physics Phenomenology, Department of Physics, Durham \\ University, Durham DH1 3LE, United Kingdom
}

\begin{abstract}
The Majorana nature of neutrinos can be experimentally verified only via lepton-number violating processes involving charged leptons. We study 36 lepton-number violating $(L V)$ processes from the decays of tau leptons and pseudoscalar mesons. These decays are absent in the Standard Model but, in presence of Majorana neutrinos in the mass range $\sim 100 \mathrm{MeV}$ to $5 \mathrm{GeV}$, the rates for these processes would be enhanced due to their resonant contribution. We calculate the transition rates and branching fractions and compare them to the current bounds from direct experimental searches for $\Delta L=2$ tau and rare meson decays. The experimental non-observation of such $L V$ processes places stringent bounds on the Majorana neutrino mass and mixing and we summarize the existing limits. We also extend the search to hadron collider experiments. We find that, at the Tevatron with $8 \mathrm{fb}^{-1}$ integrated luminosity, there could be $2 \sigma(5 \sigma)$ sensitivity for resonant production of a Majorana neutrino in the $\mu^{ \pm} \mu^{ \pm}$modes in the mass range of $\sim 10-180 \mathrm{GeV}(10-$ $120 \mathrm{GeV})$. This reach can be extended to $\sim 10-375 \mathrm{GeV}(10-250 \mathrm{GeV})$ at the LHC of 14 $\mathrm{TeV}$ with $100 \mathrm{fb}^{-1}$. The production cross section at the LHC of $10 \mathrm{TeV}$ is also presented for comparison. We study the $\mu^{ \pm} e^{ \pm}$modes as well and find that the signal could be large enough even taking into account the current bound from neutrinoless double-beta decay. The signal from the gauge boson fusion channel $W^{+} W^{+} \rightarrow \ell_{1}^{+} \ell_{2}^{+}$at the LHC is found to be very weak given the rather small mixing parameters. We comment on the search strategy when a $\tau$ lepton is involved in the final state.
\end{abstract}

\footnotetext{
*avatre@fnal.gov, than@hep.wisc.edu, silvia.pascoli@durham.ac.uk, zb@mail.tsinghua.edu.cn (Communication author)
} 


\section{Contents}

1. Introduction

2. Majorana neutrinos in extension of the standard model 5

2.1 Formalism for Heavy Neutrino Mixing 5

2.2 Current Constraints on $N_{4}$ Masses And Mixing

2.2.1 Mixing with $\nu_{e}$

2.2.2 Mixing with $\nu_{\mu}$

2.2.3 Mixing with $\nu_{\tau}$

2.2.4 Electroweak Precision Tests 10

2.2.5 Neutrinoless Double Beta Decay $(0 \nu \beta \beta)$

3. Lepton-Number Violating Decays 14

3.1 Decay Modes of Heavy Majorana Neutrino 14

3.1.1 Decay Modes of Heavy Majorana Neutrino with mass $m_{4} \ll m_{W}$

3.1.2 Decay Modes of Heavy Majorana Neutrino with mass $m_{4}>m_{W}$

3.2 Lepton-Number Violating Tau Decays 18

3.3 Lepton-Number Violating Rare Meson Decays 21

4. Collider Signatures 25

4.1 Search for Like-sign Dilepton Signals at the Tevatron 29

4.2 Search for Like-sign Dilepton Signals at the LHC 32

L.3 Like-sign Dilepton Signals with $\tau$ in the Final States 38

5. Summary and Conclusions 39

A. Lepton mixing formalism 41

B. General amplitude of $\Delta L=2$ processes

9. Decay modes of heavy Majorana neutrino 47

D. Lepton-number violating tau decay 49

E. Rare meson decay 52

\section{Introduction}

In the Standard Model (SM) of strong and electroweak interactions, neutrinos are strictly massless due to the absence of right-handed chiral states $\left(N_{R}\right)$ and the requirement of $S U(2)_{L}$ gauge invariance and renormalizability. Recent neutrino oscillation experiments have conclusively shown that neutrinos are massive [1]. This discovery presents a pressing need to consider physics beyond the SM. It is straightforward to obtain a Dirac mass 
term $m_{D}\left(\overline{\nu_{L}} N_{R}+\right.$ h.c. $)$ for a neutrino by including the right-handed state, just like the treatment for all other fermions via Yukawa couplings to the Higgs doublet in the SM. However, a profound question arises: Since $N_{R}$ is a SM gauge singlet, why should a gaugeinvariant Majorana mass term $\frac{1}{2} M N_{R} N_{R}$ not exist in the theory? In fact, there is strong theoretical motivation for the Majorana mass term to exist since it could naturally explain the smallness of the observed neutrino masses via the so-called "see-saw" mechanism [2]

$$
m_{\nu} \approx \frac{m_{D}^{2}}{M}
$$

From a model-building point of view, there are many scenarios that could incorporate the Majorana mass. Examples include Left-Right symmetric gauge theories [3]; $S O(10) \mathrm{Su}-$ persymmetric (SUSY) grand unification [4] and other grand unified theories [5]; models with exotic Higgs representations [6, 7]; R-parity violating interactions $(\Delta L=1)$ in Supersymmetry (SUSY) [8] and theories with extra dimensions [9]. There are other proposals to generate Majorana masses for neutrinos at a higher scale $M$ without relying on the right-handed state $N_{R}$ [10, 11]. According to the scheme in generating the mass scale $M$ in Eq. (1.1), it has been customary to call them Type I [2], Type II [10] or Type III [11].

Within the context of the SM, there is only one gauge-invariant operator 12 that is relevant to the neutrino mass,

$$
\frac{\kappa}{\Lambda} l_{L} H l_{L} H,
$$

where $l_{L}$ and $H$ are the SM lepton and Higgs doublets, respectively. The constant $\kappa$ is a model-dependent effective coupling and $\Lambda$ is the new physics cut-off scale. It is a dimension5 non-renormalizable operator, and leads to Majorana neutrino masses of the order $\kappa v^{2} / \Lambda$, after the Higgs field acquires a vacuum expectation value $v$, in accordance with the see-saw scheme. Higher dimensional operators that give rise to Majorana neutrino masses have also been constructed in a model-independent manner [13]. The challenging task is to look for experimental evidence to probe the new physics scale $\Lambda$ and to distinguish the underlying theoretical models mentioned above.

In the neutrino sector, besides the rich phenomena of neutrino flavor oscillations and the possible existence of new sources of $\mathrm{CP}$-violation, lepton number violation by two units $(\Delta L=2)$, as implied by a Majorana mass term, plays a crucial role. Not only may it result in important consequences in particle physics, nuclear physics and cosmology but it would also guide us in understanding the fundamental symmetries of physics beyond the SM. Although the prevailing theoretical prejudice prefers Majorana neutrinos, experimentally testing the nature of neutrinos and lepton-number violation $(L V)$ in general, is of fundamental importance. In accelerator-based experiments, neutrinos in the final state are undetectable by the detectors, leading to the so-called "missing energy" and therefore missing lepton numbers as well. One is thus forced to look for charged leptons in the final state. The basic process with $\Delta L=2$ can be generically expressed by

$$
W^{-} W^{-} \rightarrow \ell_{1}^{-} \ell_{2}^{-},
$$

where $W^{-}$is a virtual SM weak boson and $\ell_{1,2}=e, \mu, \tau$. By coupling fermion currents to the $W$ bosons as depicted in Fig. 1, and arranging the initial and final states properly, one finds various physical processes that can be experimentally searched for. The best known example is neutrinoless double-beta decay $(0 \nu \beta \beta)$ 14, 15, 16], which proceeds via the parton-level subprocess $d d \rightarrow u u W^{-*} W^{-*} \rightarrow u u e^{-} e^{-}$. Other interesting classes 


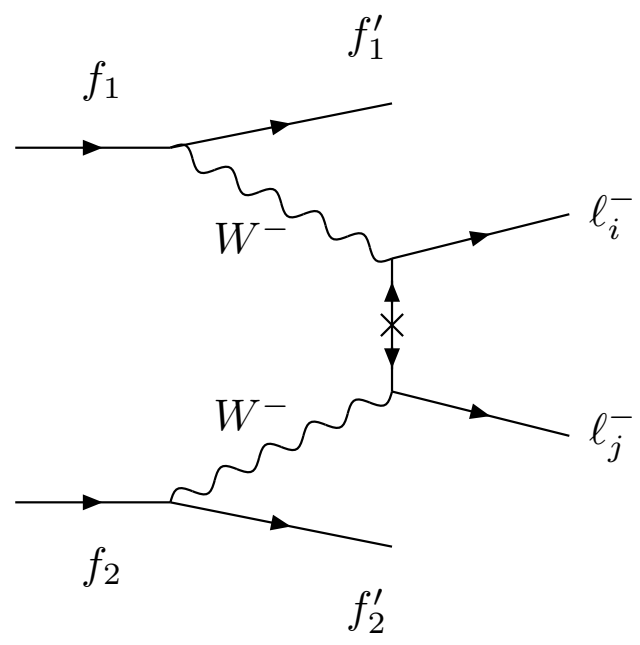

Figure 1: A generic diagram for $\Delta L=2$ processes via Majorana neutrino exchange.

of $L V$ processes involve tau decays such as $\tau^{-} \rightarrow \ell^{+} M_{1}^{-} M_{2}^{-}$[17, 18] where the light mesons $M_{1}, M_{2}$ are $\pi, K$, rare meson decays such as $M_{1}^{+} \rightarrow \ell_{1}^{+} \ell_{2}^{+} M_{2}^{-}$[19, 20, 18] and hyperon decays such as $\Sigma^{-} \rightarrow \Sigma^{+} e^{-} e^{-}, \Xi^{-} \rightarrow p \mu^{-} \mu^{-}$etc. [21]. One could also explore additional processes like $e^{-} \rightarrow \mu^{+}$[22], $\mu^{-} \rightarrow e^{+}$[18, 23] and $\mu^{-} \rightarrow \mu^{+}$conversion [18, 24]. One may also consider searching for signals at accelerator and collider experiments via $e^{-} e^{-} \rightarrow W^{-} W^{-}$[25], $e^{+} e^{-} \rightarrow Z^{0} \rightarrow N+X$ [26], $e^{ \pm} p \rightarrow \nu_{e}\left(\overline{\nu_{e}}\right) \ell_{1}^{ \pm} \ell_{2}^{ \pm} X$ [27], neutrino nucleon scattering $\nu_{\ell}\left(\overline{\nu_{\ell}}\right) \mathcal{N} \rightarrow \ell^{\mp} \ell_{1}^{ \pm} \ell_{2}^{ \pm} X$ [28], $p p \rightarrow \ell_{1}^{+} \ell_{2}^{+} X$ [29, 30, 31, 32, 33, 34], topquark decays $t \rightarrow b \ell_{1}^{+} \ell_{2}^{+} W^{-}$[35], charged-Higgs production $e^{ \pm} e^{ \pm} \rightarrow H^{ \pm} H^{ \pm}$[36], and in the decay $N \rightarrow \ell^{ \pm} H^{\mp}$ [37].

The dynamics for $\Delta L=2$ processes as in Eq. (1.3) is dictated by the properties of the exchanged neutrinos. For a Majorana neutrino that is light compared to the energy scale in the process, the transition rates for $L V$ processes are proportional to the product of two flavor mixing matrix elements among the light neutrinos and a $L V$ mass insertion

$$
\langle m\rangle_{\ell_{1} \ell_{2}}^{2}=\left|\sum_{m=1}^{3} U_{\ell_{1} m} U_{\ell_{2} m} m_{\nu_{m}}\right|^{2},
$$

where $\langle m\rangle_{\ell_{1} \ell_{2}}$ are the "effective neutrino masses". If the neutrinos are heavy compared to the energy scale involved, then the contribution scales as

$$
\left|\sum_{m^{\prime}=4}^{3+n} \frac{V_{\ell_{1} m^{\prime}} V_{\ell_{2} m^{\prime}}}{m_{N_{m^{\prime}}}}\right|^{2},
$$

where $V$ is the mixing matrix between the light flavor and heavy neutrinos. Unfortunately, both situations encounter a severe suppression either due to the small neutrino mass like $m_{\nu_{m}}^{2} / M_{W}^{2}$, or due to the small mixing $\left|V_{\ell_{1} m^{\prime}} V_{\ell_{2} m^{\prime}}\right|^{2}$. An important observation is that when the heavy neutrino mass is kinematically accessible, a process may undergo resonant production of the heavy neutrino. The transition rate can be substantially enhanced and goes like

$$
\frac{\Gamma\left(N_{m^{\prime}} \rightarrow i\right) \Gamma\left(N_{m^{\prime}} \rightarrow f\right)}{m_{N_{m^{\prime}}} \Gamma_{N_{m^{\prime}}}},
$$


where $i, f$ refer to the initial and final state during the transition.

The possible existence of sterile neutrinos in the mass range relevant for resonant enhancement of $\Delta L=2$ processes studied in this paper is motivated in several scenarios. Models which implement the see-saw mechanism at low energies have been recently considered [38, 39]: the neutrino masses generated are accidentally small and active-sterile mixing can be as large as few percent. See-saw models at the electroweak scale can explain neutrino masses if appropriate symmetries are imposed and at the same time provide an appealing mechanism for baryon asymmetry generation via resonant leptogenesis [40]. In theories with dynamical electroweak symmetry breaking, sterile neutrinos with masses in the $100 \mathrm{~s}$ of $\mathrm{MeV}$ to $\mathrm{GeV}$ range are invoked to explain light neutrino masses [41]. Sterile neutrinos can also play a role in understanding the flavour problem in the leptonic sector. It has been shown that mixing with sterile neutrinos can be at the origin of the large angles in the neutrino sector 42.

Heavy, mostly-sterile neutrinos have been investigated for their role in cosmology and astrophysics, in particular in Big Bang Nucleosynthesis, Large Scale Structure formation [43], cosmic microwave background, diffuse extragalactic background radiation, supernovae [44] and as dark matter candidates [45, 46, 47] (for a review on $\mathrm{MeV}$ sterile neutrinos, see Ref. [48]). A keV sterile neutrino is a viable dark matter candidate [45, 47], which can also explain the origin of pulsar kicks [49]. Decays of heavy, mostlysterile neutrinos have been proposed to explain the early ionization of the Universe [50]. Due to mixing, dark matter sterile neutrinos would decay radiatively contributing to the Diffuse Extragalactic Background Radiation and inducing x-ray emission from galaxy clusters [51, 52]. A large coupling between sterile neutrinos and light dark matter scalars can be at the origin of neutrino masses and of the observed dark matter abundance [53]. A model with sterile neutrinos in the $\mathrm{keV}-\mathrm{GeV}$ mass range has been proposed to explain the dark matter of the Universe as well as baryogenesis [54, 55]. Its phenomenological and astrophysical signatures have been considered in detail in Refs. [56, 52]. This model assumes the existence of one sterile neutrino with $\mathrm{keV}$ mass for dark matter and two heavier neutrinos with quasi-degenerate $\mathrm{GeV}$ masses for successful baryogenesis. The required mixing $\left|V_{\ell m^{\prime}}\right|^{2}$ of the latter neutrinos with the active ones is mass dependent and lies in the range $10^{-11}-10^{-8}$, for a mass of $1 \mathrm{GeV}$. Additional constraints on the heavy neutrino mass and mixing angles can be derived from astrophysical observations. Sterile neutrinos mixed with active ones would be efficiently produced in supernovae cores, escaping from it and depleting substantially the supernova core energy, and, therefore, might modify the supernova evolution. Recently, it was shown that sterile neutrinos in the mass range $\sim 0.2 \mathrm{GeV}$ and small mixing angle with $\mu$ and $\tau$ neutrinos could enhance the energy transport from the core to the stalled shock and favor the supernova explosion 44. They could also explain the high velocity of pulsars if the momentum carried away by heavy sterile neutrinos is emitted asymmetrically 449. Detailed reviews and discussions of heavy neutrinos in the Early Universe and their present bounds can be found, e.g., in Refs. [48, 57, 58].

Cosmological and astrophysical constraints on sterile neutrinos are typically very strong but are not as robust as the ones from laboratory searches as they typically depend on the production mechanism of sterile neutrinos in the Early Universe and on the cosmological evolution. For example, they can be significantly weakened or evaded if the reheating temperature is low 477, 59, if their density in the Early Universe is diluted by entropy injected at late times [55] or if they have non-standard interactions. In these cases, much larger mixing angles with active neutrinos are allowed by cosmological observations and 
can be tested in terrestrial experiments. Therefore, it is important to perform experimental searches of heavy sterile neutrinos with increased sensitivity and, specifically for Majorana neutrinos, to consider $\Delta L=2$ processes. If a positive signal is found and is incompatible with the cosmological and/or astrophysical observations, one would need to consider modifications to the standard cosmological scenario and/or would gain new insight on the evolution of astrophysical objects.

In this paper, we study resonant contributions of heavy Majorana neutrinos to $\Delta L=2$ processes involving two charged leptons in accelerator-based experiments. We establish our conventions and discuss the current constraints on the mass and mixing of heavy neutrinos in Sec. 2. In Section 3 we lay out the general expressions for the heavy neutrino contributions to low energy $L V$ decays and study two classes of $\Delta L=2$ processes,

(a) tau decays $\tau^{-} \rightarrow \ell^{+} M_{1}^{-} M_{2}^{-}$,

(b) rare meson decays $K^{+}, D^{+}, D_{s}^{+}, B^{+} \rightarrow \ell_{1}^{+} \ell_{2}^{+} M_{2}^{-}$.

We calculate the enhanced transition rates and branching fractions and compare them to the bounds set by direct experimental searches. A non-observation of such $\Delta L=2$ processes places stringent constraints on the mass and mixing of Majorana neutrinos which are also presented in this section. The resonant production of Majorana neutrinos at hadron colliders, namely the Tevatron and LHC are studied and updated in Sec. 4 . We draw our conclusions in Sec. 5. We discuss in detail the formalism, the decay modes and the total decay width of heavy Majorana neutrinos and the transition rates of $L V$ processes in the Appendices.

\section{Majorana neutrinos in extension of the standard model}

To set up our notation and convention, we first discuss the formalism for the simplest extension of the SM which includes right handed singlets. Also in this section, we present the current constraints on the mass and mixing of a heavy neutrino from various direct detection experiments, accelerator searches and electroweak precision constraints.

\subsection{Formalism for Heavy Neutrino Mixing}

The leptonic content in our simplest extension of the SM includes three generations of left-handed SM $S U(2)_{L}$ doublets and $n$ right-handed SM singlets

$$
L_{a L}=\left(\begin{array}{c}
\nu_{a} \\
l_{a}
\end{array}\right)_{L}, \quad N_{b R},
$$

where $a=1,2,3$ and $b=1,2, \cdots, n$. The gauge-invariant Yukawa interactions lead to Dirac masses for the charged leptons and neutrinos after the Higgs field develops a vacuum expectation value $v$. It is also possible for the singlet neutrinos to have a heavy Majorana mass term. The full neutrino mass terms as well as the diagonalized eigenvalues can be expressed as

$$
\begin{aligned}
-\mathcal{L}_{m}^{\nu} & =\frac{1}{2}\left(\sum_{a=1}^{3} \sum_{b=1}^{n}\left(\overline{\nu_{a L}} m_{a b}^{\nu} N_{b R}+\overline{N_{b L}^{c}} m_{b a}^{\nu *} \nu_{a R}^{c}\right)+\sum_{b, b^{\prime}=1}^{n} \overline{N_{b L}^{c}} B_{b b^{\prime}} N_{b^{\prime} R}\right)+\text { h.c. } \\
& =\frac{1}{2}\left(\sum_{m=1}^{3} m_{\nu_{m}} \overline{\nu_{m L}} \nu_{m R}^{c}+\sum_{m^{\prime}=4}^{3+n} M_{N_{m^{\prime}}} \overline{N_{m^{\prime} L}^{c}} N_{m^{\prime} R}\right)+\text { h.c. }
\end{aligned}
$$


with the mixing relations between the gauge and mass eigenstates

$$
\begin{aligned}
& \nu_{a L}=\sum_{m=1}^{3} U_{a m} \nu_{m L}+\sum_{m^{\prime}=4}^{3+n} V_{a m^{\prime}} N_{m^{\prime} L}^{c}, \\
& U U^{\dagger}+V V^{\dagger}=I .
\end{aligned}
$$

In terms of the mass eigenstates, the gauge interaction Lagrangian can be written as

$$
\begin{aligned}
-\mathcal{L} & =\frac{g}{\sqrt{2}} W_{\mu}^{+}\left(\sum_{\ell=e}^{\tau} \sum_{m=1}^{3} U_{\ell m}^{*} \overline{\nu_{m}} \gamma^{\mu} P_{L} \ell+\sum_{\ell=e}^{\tau} \sum_{m^{\prime}=4}^{3+n} V_{\ell m^{\prime}}^{*} \overline{N_{m^{\prime}}^{c}} \gamma^{\mu} P_{L} \ell\right)+\text { h.c. } \\
& +\frac{g}{2 \cos \theta_{W}} Z_{\mu}\left(\sum_{\ell=e}^{\tau} \sum_{m=1}^{3} U_{\ell m}^{*} \overline{\nu_{m}} \gamma^{\mu} P_{L} \nu_{\ell}+\sum_{\ell=e}^{\tau} \sum_{m^{\prime}=4}^{3+n} V_{\ell m^{\prime}}^{*} \overline{N_{m^{\prime}}^{c}} \gamma^{\mu} P_{L} \nu_{\ell}\right)+\text { h.c. (2.5) }
\end{aligned}
$$

Further details about the mixing formalism are given in Appendix A.

A few important remarks are in order before the detailed considerations. First of all, parameterically, the light neutrino masses $m_{\nu, \text { diag }}$ are of the order of magnitude $\left(m_{D}^{\nu}\right)^{2} / B$, while the heavy neutrino masses are $M_{N \text {, diag }} \simeq B$. Secondly, the mixing parameters would typically scale as $U^{\dagger} U \approx I$ and $V^{\dagger} V \approx m_{\nu} / M_{N}$. Thirdly, the Majorana mass term for the flavor states $\nu_{a L}$, absent in Eq. (2.2) and corresponding to the null entry $0_{3 \times 3}$ in Eq. (A.8), may receive non-zero contributions as Majorana masses for the light active neutrinos, for instance from higher dimensional $\Delta L=2$ operators or in theories with a triplet Higgs field. The general formalism presented here remains the same. In this paper, we will take a phenomenological approach toward these parameters. We will simply take the masses and mixing elements of the heavy neutrino as free parameters, only subject to some constraints from experimental observations. The assumption that the masses and mixing elements are not rigorously related by the see-saw relations is feasible from a model-building point of view, since some fine-tuning or some ansatz of the neutrino mass matrix can always alter the general relations. Several scenarios where it is possible to have rather low mass of the heavy neutrino were mentioned in the previous section. Here and henceforth, we consider the case when only one heavy Majorana neutrino is kinematically accessible and denote it by $N_{4}$, with the corresponding mass $m_{4}$ and mixing with charged lepton flavors $V_{\ell 4}$. If we stick with this simple parameterization, the SM Higgs boson will couple to the heavy neutrinos as well. We present the couplings in Appendix A. When appropriate, we will include this effect. As noted above, some fine-tuning [60] would be needed to avoid excessive contributions to the light neutrino mass.

\subsection{Current Constraints on $N_{4}$ Masses And Mixing}

In laboratory searches, no positive evidence of sterile neutrinos has been found so far,$^{1}$ in the mass range of interest, $100 \mathrm{eV}-100 \mathrm{GeV} .^{2}$

\footnotetext{
${ }^{1}$ Indications of the existence of a neutrino with $17 \mathrm{keV}$ mass were subsequently shown to be non valid. For a review, see Ref. 61. Studying interactions of neutrinos from $\pi$ and $\mu$ decays, an anomaly in time distribution was found [62]. It could be interpreted as the existence of a neutrino emitted in pion decays with mass of $33.9 \mathrm{MeV}$. Searches for this neutral fermion have not given any positive signature and have allowed to constrain the mixing to be $\left|V_{\mu 4}\right|^{2}<9.2 \times 10^{-8}$ at $95 \%$ C.L. 63.

${ }^{2}$ For sterile neutrinos with smaller masses a rather complete analysis of the bounds can be found in Ref. 64]. See also the implications of the recent results from the MiniBooNE collaboration [65, 66].
} 
A very powerful probe of the mixing of heavy neutrinos with both $\nu_{e}$ and $\nu_{\mu}$ are peak searches in leptonic decays of pions and kaons 67. If a heavy neutrino is produced in such decays, the lepton spectrum would show a monochromatic line at

$$
E_{\ell}=\frac{m_{M}^{2}+m_{\ell}^{2}-m_{4}^{2}}{2 m_{M}}
$$

where $E_{\ell}$ and $m_{\ell}$ are respectively the lepton energy and mass, $m_{M}$ is the meson mass. The mixing angle controls the branching ratio of this process as:

$$
\frac{\Gamma\left(M^{+} \rightarrow \ell^{+} N_{4}\right)}{\Gamma\left(M^{+} \rightarrow \ell^{+} \nu_{\ell}\right)}=\frac{\left|V_{\ell 4}\right|^{2}}{\sum_{m=1}^{3}\left|U_{\ell m}\right|^{2}} \rho \approx\left|V_{\ell 4}\right|^{2} \rho,
$$

where $\rho$ is a kinematical factor 67:

$$
\rho=\frac{\sqrt{1+\mu_{\ell}^{2}+\mu_{4}^{2}-2\left(\mu_{\ell}+\mu_{4}+\mu_{\ell} \mu_{4}\right)}\left(\mu_{\ell}+\mu_{4}-\left(\mu_{\ell}-\mu_{4}\right)^{2}\right)}{\mu_{\ell}\left(1-\mu_{\ell}\right)^{2}},
$$

with $\mu_{i}=m_{i}^{2} / m_{M}^{2}$. For large $m_{4}$, the helicity suppression of the $\pi, K \rightarrow \ell \nu_{\ell}$ decays weakens and there is an enhancement for $M^{+} \rightarrow \ell^{+} N_{4}$ by a relative factor $m_{4}^{2} / m_{\ell}^{2}$, reaching up to $10^{4}-10^{5}$ compared to that of $\pi \rightarrow e \nu_{e}$ and $K \rightarrow e \nu_{e}$ in the SM, respectively. These bounds are very robust because they rely only on the assumption that a heavy neutrino exists and mixes with $\nu_{e}$ and/or $\nu_{\mu}$.

Another strategy to constrain heavy neutrinos mixed with $\nu_{e}, \nu_{\mu}$ and $\nu_{\tau}$, is via searches of the products of their decays. If kinematically allowed, $N_{4}$ would be produced in every process in which active neutrinos are emitted with a branching fraction proportional to the mixing parameter $\left|V_{\ell 4}\right|^{2}$. They would subsequently decay via Charged Current (CC) and Neutral Current (NC) interactions into neutrinos and other "visible" particles, such as electrons, muons and pions. Searches for these "visible" decay-products were performed and were used to constrain the mixing parameters. In beam dump experiments, $N_{4}$ would be produced in meson decays, with the detector located far away from the production site. The suppression of the flux of $N_{4}$ needs to be taken into account if the decay length is very short and, therefore, typically both an upper and a lower bound on the mixing angle can be set. Otherwise, the production can happen in the detector itself, as for the limits obtained from a reanalysis of LEP data, using the possible decays of the $Z^{0}[26$. into heavy neutrinos. In this case, large values of the mixing angle can be excluded. These bounds are less robust than the ones previously discussed. In fact, if the heavy neutrinos have other dominant decay channels into invisible particles, these bounds would be weakened, if not completely evaded. For example, a coupling of the type $g N \nu \phi$ (see Ref. 68, 53]), with $\phi$ a scalar, can induce very fast decays, which might dominate over the ones induced by $\mathrm{CC}$ and $\mathrm{NC}$ interactions. In this case, if the decay length is very short due to these strong interactions, the flux of $N_{4}$ might be suppressed at the far detector and the bound would not apply. If the production happens in the detector itself, the bounds would need to be recomputed considering the branching fraction into "visible" channels. Notice that we do not report here the bounds from Ref. [69] as they do not apply to the heavy neutrinos under consideration. In these analyses it was assumed that heavy neutrinos were produced via $Z^{0} \rightarrow N_{4} \bar{N}_{4}$ with the same strength as an active neutrino. In our scheme, this would correspond to having mixing angle equal to 1 . Then, the search for $N_{4}$ decays 
in the detector was used to constrain the heavy neutrino parameters. These data should be reanalyzed considering that the production of $N_{4}$ is suppressed by $\left|V_{\ell 4}\right|^{2}$. Comparing the expected number of events with the backgrounds we estimate that typically bounds of order $\left|V_{\ell 4}\right|^{2}<$ few $\times 10^{-3}-10^{-2}$ could be deduced. However, a detailed analysis should be performed and we do not report these limits in our figures.

For masses above the production threshold, additional constraints can be obtained from lepton universality as the decay rates for muons, pions, taus as well as the invisible decay width for the $Z^{0}$ boson are modified with respect to the SM predictions [70, 71, 72]. Flavour changing neutral current processes such as $\mu \rightarrow e \gamma, \mu \rightarrow e e^{+} e^{-}$and $\mu-e$ conversion in nuclei are affected by the existence of heavy sterile neutrinos and strong limits can be obtained on the mixing with active neutrinos [73, 74, 75]. These bounds are reported in Section 2.2.4.

Finally, in Section 2.2.5 we discuss the very strong constraints on $\left|V_{e 4}\right|^{2}$ which can be obtained from the non-observation of neutrinoless double beta decay. It should be noticed that in the presence of more than one sterile neutrino, possible cancellations between the contributions to the decay rate can be achieved and the bounds would be consequently much weaker.

Next, we review the laboratory constraints on the mixing between heavy and active neutrinos, depending on flavour and the mass of sterile neutrinos.

\subsubsection{Mixing with $\nu_{e}$}

The mixing parameter $V_{e 4}$ can be tested in searches of kinks in the $\beta$-decay spectrum, of peaks in the spectrum of electrons in meson decays and, finally, of $N_{4}$ decays in reactor and accelerator neutrino experiments.

For masses $30 \mathrm{eV} \simeq m_{4} \simeq 1 \mathrm{MeV}$, the most sensitive probe is the search for kinks in the $\beta$-decay spectra [67. In the presence of heavy neutrinos mixed with $\nu_{e}$, the Kurie plot would be given by the contributions of the decays into light neutrinos as well as into heavy ones. This induces a kink in the Kurie plot at the end point electron energy $E_{e}$

$$
E_{e}=\frac{M_{i}^{2}+m_{e}^{2}-\left(M_{f}+m_{4}\right)^{2}}{2 M_{i}}
$$

where $M_{i, f}$ are the mass of the initial and final nuclei, respectively, and $m_{e}$ is the electron mass. In Fig. 2 we report the most stringent limits, obtained by using different nuclei [76, 77, 78, 79, 80]. In reactors and in the Sun only low mass, $m_{4}<$ few $\mathrm{MeV}$, heavy neutrinos can be produced. The constraints obtained by looking for their decays into electron-positron pairs [81, 82] are reported in Fig. 2 with solid (cyan) contour labeled Bugey and short dashed (blue) contour labeled Borexino. The region with long dash dotted (grey) contour, labelled $\pi \rightarrow e \nu$, is excluded by peak searches [83].

For heavier masses peak searches give the most stringent bounds, shown in Fig. 3 . Notice that, due to the weakened helicity suppression of the $\pi$ decay, the sensitivity on $V_{e 4}$ increases with $m_{4}$ till phase space becomes relevant at $m_{4}>80 \mathrm{MeV}$, for $\pi \rightarrow e \nu_{e}$. The excluded region, at 90\% C.L., from Ref. [83], is indicated with the solid (black) line labeled $\pi \rightarrow e \nu$. For heavier masses, stringent bounds are obtained by looking at the electron spectrum in $K$ decays 85] and are indicated by the double dash dotted (purple) line labeled $K \rightarrow e \nu$ in Fig. 3. Assuming that only CC and NC interactions are at play, stringent bounds have been obtained on $\left|V_{e 4}\right|^{2}$ and are reported in Fig. 3 by the rest of the contours (except dotted (maroon) line labeled $0 \nu \beta \beta$ ). In particular, the limits at $90 \%$ C.L. 


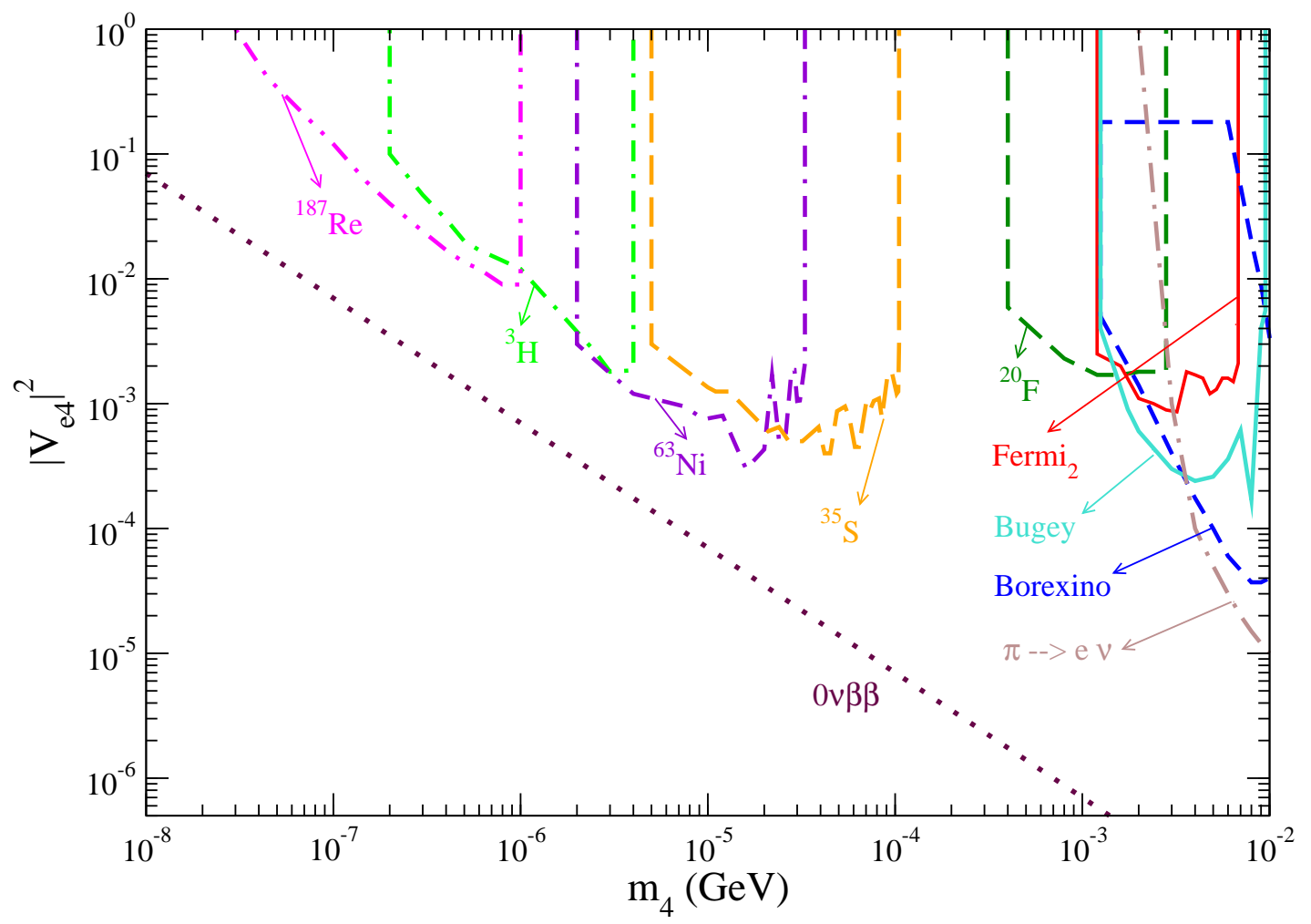

Figure 2: Bounds on $\left|V_{e 4}\right|^{2}$ versus $m_{4}$ in the mass range $10 \mathrm{eV}-10 \mathrm{MeV}$. The excluded regions with contours labeled ${ }^{187} \mathrm{Re}\left[76,{ }^{3} \mathrm{H}\left[77,{ }^{63} \mathrm{Ni} 78\right],{ }^{35} \mathrm{~S}[79],{ }^{20} \mathrm{~F}\right.$ and $\mathrm{Fermi}_{2}$ 80] refer to the bounds from kink searches. All the limits are given at 95\% C.L. except for the ones from Ref. 80 which are at $90 \%$ C.L.. The areas delimited by short dashed (blue) contour labeled Borexino and solid (cyan) contour labeled Bugey are excluded at $90 \%$ C.L. by searches of $N_{4}$ decays from the Borexino Counting Test facility 81 and Ref. [82 respectively. The region with long-dash-dotted (grey) contour, labelled $\pi \rightarrow e \nu$, is excluded by peak searches [83]. The dotted (maroon) line labeled $0 \nu \beta \beta$ indicates the bound from searches of neutrinoless double beta-decay [84].

from Refs. [86, 87, 88], assume the production of $N_{4}$ in meson decays and look for visible channels in a detector located some distance from the source. The limits at 95\% C.L. in Refs. 89, 90] analyse the data from DELPHI and L3 detectors, looking for $N_{4}$ from $Z^{0}$ decays. In Fig. 3 we also report the excluded region from neutrinoless double beta-decay experiments [91, 84], bounded by dotted (maroon) line, valid if the heavy neutrinos are Majorana particles (see further).

\subsubsection{Mixing with $\nu_{\mu}$}

The bounds on $\left|V_{\mu 4}\right|^{2}$ come from searches of peaks in the spectrum of muons in pion and kaon decays and of the decays of $N_{4}$ produced in neutrino beams and $e^{+} e^{-}$collisions.

As already discussed in the case of mixing with $\nu_{e}$, peak searches provide very robust and stringent bounds, by looking at pion decays for masses up to $34 \mathrm{MeV}$, and at kaon decays for higher masses. A detailed review is given in Figs. 1 and 2 in Ref. 92] and for masses larger than $100 \mathrm{MeV}$ the limits are reported in Fig. 1 . The other limits on $\left|V_{\mu 4}\right|^{2}$ are found in decay searches and are also shown in Fig. 1 . They come from beam dump experiments [87, 86, 93, 94, 95] and from direct $N_{4}$ production in the detectors 


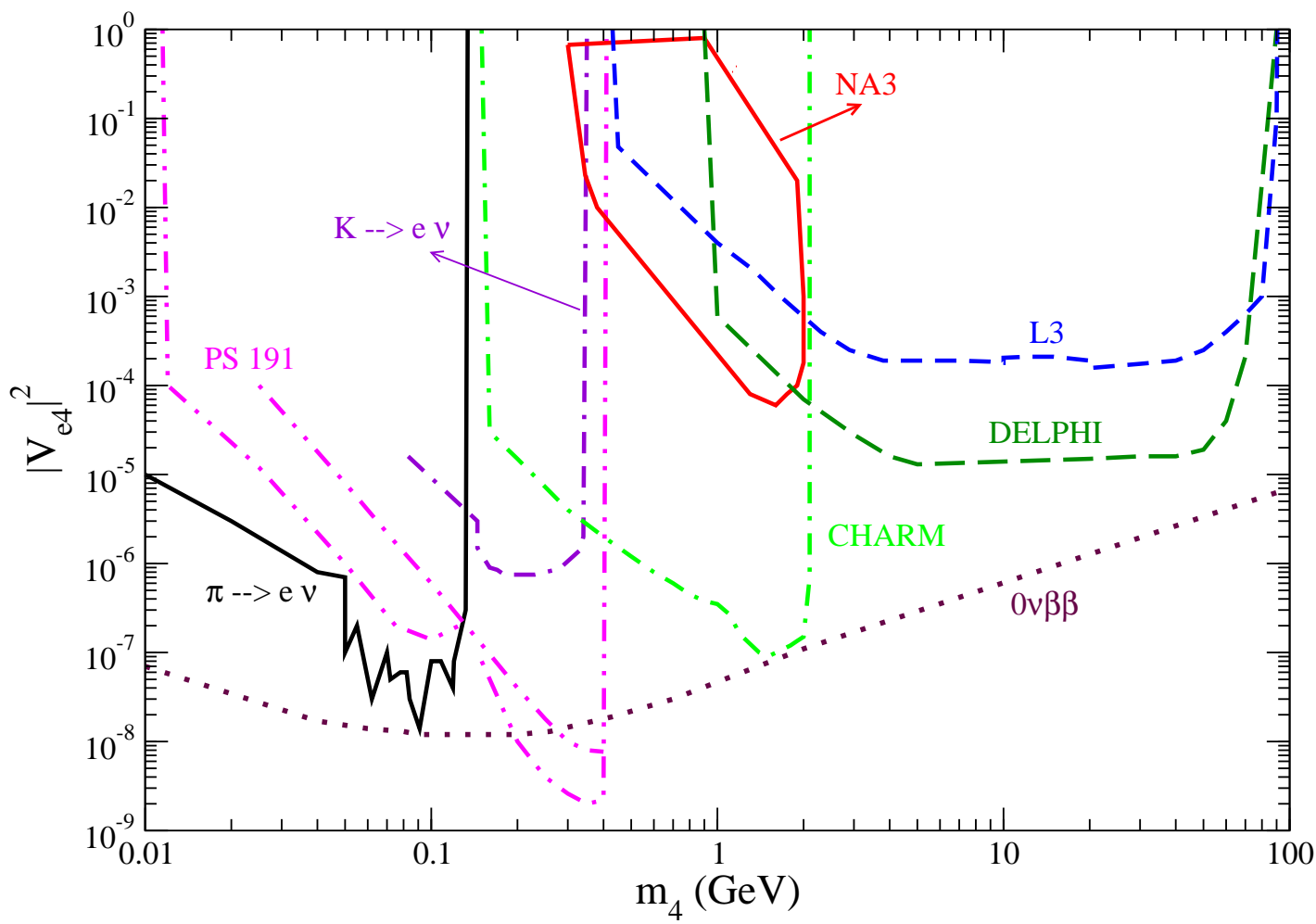

Figure 3: Bounds on $\left|V_{e 4}\right|^{2}$ versus $m_{4}$ in the mass range $10 \mathrm{MeV}-100 \mathrm{GeV}$. The areas with solid (black) contour labeled $\pi \rightarrow e \nu$ and double dash dotted (purple) contour labeled $K \rightarrow e \nu$ are excluded by peak searches 83, 85. Limits at 90\% C.L. from beam-dump experiments are taken from Ref. 86] (PS191), Ref. 87] (NA3) and Ref. 88] (CHARM). The limits from contours labeled DELPHI and L3 are at 95\% C.L. and are taken from Refs. [89] and [90] respectively. The excluded region with dotted (maroon) contour is derived from a reanalysis of neutrinoless double beta decay experimental data [84].

\section{DELPHI [89, L3 [90] and CHARM [96].}

\subsubsection{Mixing with $\nu_{\tau}$}

Heavy neutrinos mixed with $\tau$ neutrinos can be produced either via $\mathrm{CC}$ interactions if a $\tau$ is produced or in $\mathrm{NC}$ interactions. The only limits come from searches of $N_{4}$ decays and are reported in Fig. 5. The bounds at 90\% C.L. from CHARM 97 and NOMAD 98 assume production via $D$ and $\tau$ decays. The DELPHI bound at 95\% C.L. 89 assumes $N_{4}$ production in $Z^{0}$ decays and with respect to the bound on $\left|V_{e 4}\right|^{2}$ and $\left|V_{\mu 4}\right|^{2}$ there is $\tau$ production kinematical suppression for low masses which weakens the constraint for masses in the range $m_{4} \sim 2-3 \mathrm{GeV}$.

\subsubsection{Electroweak Precision Tests}

The presence of heavy neutral fermions affects processes below their mass threshold due to their mixing with standard neutrinos [70] and significant bounds can be set by precision electroweak data. The effective $\mu$-decay constant $G_{\mu}$, measured in muon decays, is modified with respect to the SM value and can be related to the fundamental coupling $G_{F}$ as:

$$
G_{\mu}=G_{F} \sqrt{\left(1-\left|V_{e 4}\right|^{2}\right)\left(1-\left|V_{\mu 4}\right|^{2}\right)} .
$$




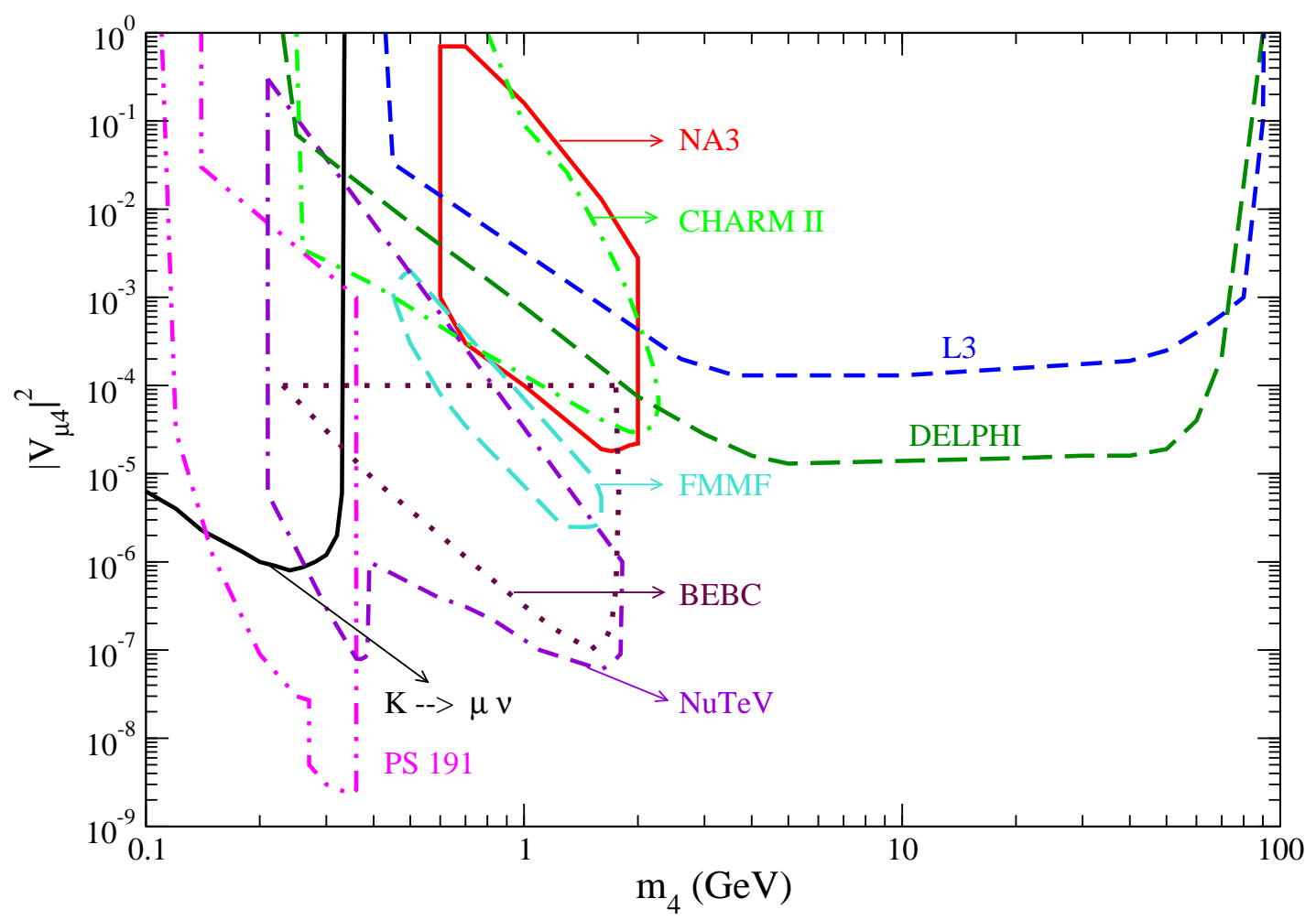

Figure 4: Limits on $\left|V_{\mu 4}\right|^{2}$ versus $m_{4}$ in the mass range $100 \mathrm{MeV}-100 \mathrm{GeV}$ come from peak searches and from $N_{4}$ decays. The area with solid (black) contour labeled $K \rightarrow \mu \nu \sqrt[92]{ }$ is excluded by peak searches. The bounds indicated by contours labeled by PS191 [86], NA3 87], BEBC [93, FMMF 94], NuTeV 95 and CHARMII [96] are at 90\% C.L., while DELPHI 89] and L3 99] are at $95 \%$ C.L. and are deduced from searches of visible products in $N_{4}$ decays. For the beam dump experiments, NA3, PS191, BEBC, FMMF and NuTeV we give an estimate of the upper limit for the excluded values of the mixing angle.

The $\mu-e$ universality test, done by comparing the decay rate of pions into $e \bar{\nu}$ and $\mu \bar{\nu}$, can be used to constrain the ratio

$$
\frac{1-\left|V_{e 4}\right|^{2}}{1-\left|V_{\mu 4}\right|^{2}}
$$

for $m_{4}>m_{\pi}[70,71]$. The analysis of experimental data leads to $\frac{1-\left|V_{\mu 4}\right|^{2}}{1-\left|V_{e 4}\right|^{2}}=1.0012 \pm 0.0016$ [71], which implies $\left|V_{e 4}\right|^{2}<0.004$ at $2 \sigma$ for the least conservative case of $\left|V_{\mu 4}\right|^{2}=0$. For $m_{4}>m_{\tau}$, the $\mu-\tau$ universality sets limits on:

$$
\frac{1-\left|V_{\tau 4}\right|^{2}}{1-\left|V_{\mu 4}\right|^{2}}
$$

and can be tested by looking at the $\tau$ leptonic and hadronic decays which give $\left|V_{\tau 4}\right|^{2}-$ $\left|V_{\mu 4}\right|^{2}=0.0057 \pm 0.0065$ [71] and $\left|V_{\tau 4}\right|^{2}-\left|V_{e 4}\right|^{2}=0.0054 \pm 0.0064$ [71]. The most constraining bound on $\left|V_{\tau 4}\right|^{2}$ is obtained for $\left|V_{e 4}\right|^{2},\left|V_{\mu 4}\right|^{2}=0$ and reads $\left|V_{\tau 4}\right|^{2}<0.018$ at $2 \sigma$. The unitarity constraint on the first row of the CKM matrix [99] reads

$$
\sum_{i=1,2,3}\left|V_{u i}^{\mathrm{CKM}}\right|^{2}=\frac{1}{1-\left|V_{\mu 4}\right|^{2}}=0.9992 \pm 0.0011
$$




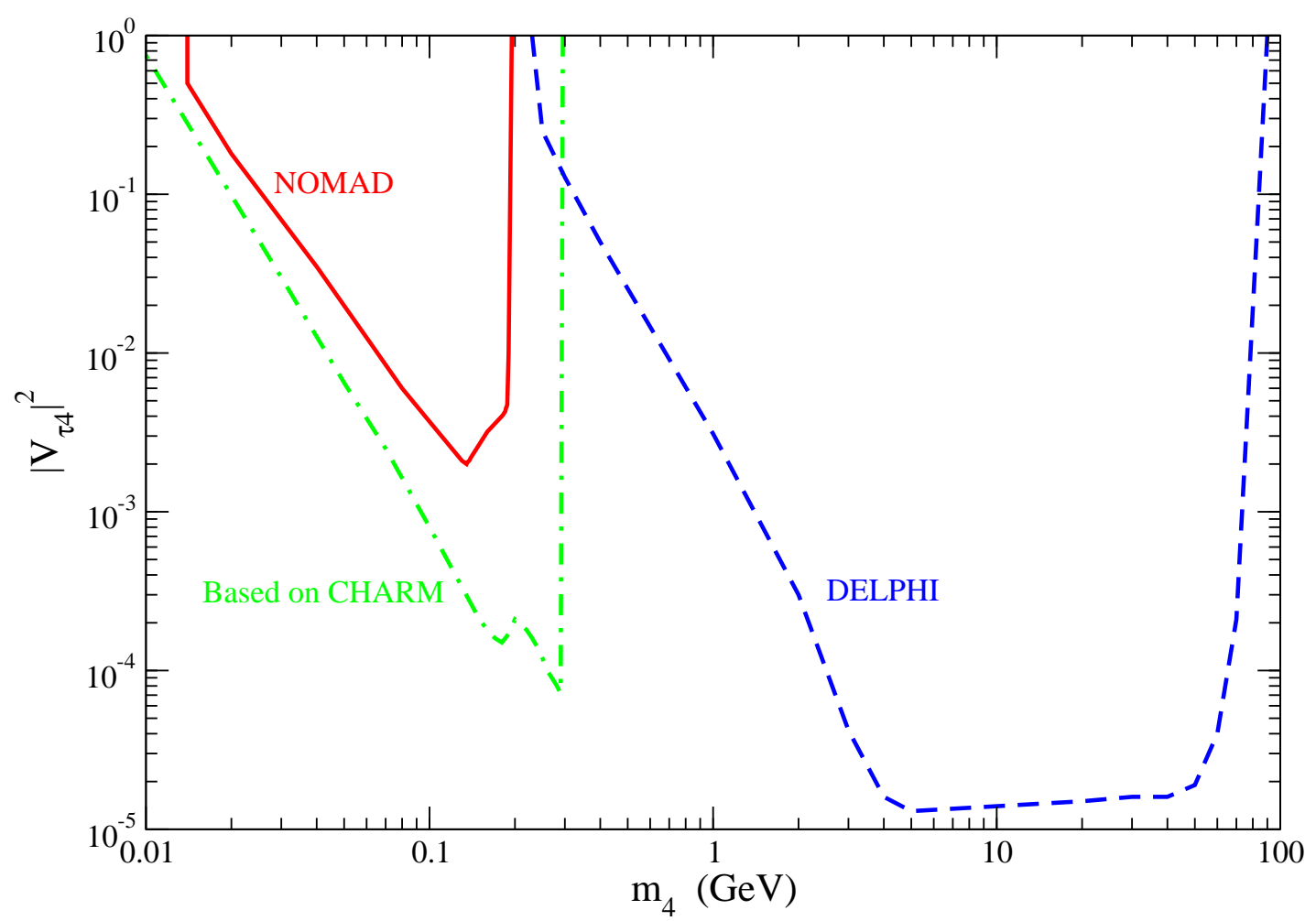

Figure 5: Bounds on $\left|V_{\tau 4}\right|^{2}$ versus $m_{4}$ from searches of decays of heavy neutrinos, given in Ref. [97] (CHARM) and in Ref. [98] (NOMAD) at 90\% C.L., and in Ref. 89] (DELPHI) at 95\% C.L.

and translates into a very strong bound on $\left|V_{\mu 4}\right|^{2},\left|V_{\mu 4}\right|^{2}<0.0003(0.0014)$, at $1(2) \sigma$, which holds for sterile neutrinos heavier than the $\Lambda$ baryon.

In the presence of heavy singlet neutrinos heavier than half the $Z^{0}$ mass, the invisible decay rate of $Z^{0}$ would be reduced with respect to the SM one, $\Gamma_{Z \rightarrow \text { inv }}^{\mathrm{SM}}$, as:

$$
\frac{\Gamma_{Z \rightarrow \text { inv }}}{\Gamma_{Z \rightarrow \text { inv }}^{\text {SM }}} \simeq\left(1-\frac{1}{6}\left|V_{e 4}\right|^{2}-\frac{1}{6}\left|V_{\mu 4}\right|^{2}-\frac{2}{3}\left|V_{\tau 4}\right|^{2}\right)
$$

By a standard model fit to LEP data, the effective number of neutrinos is now determined to be $N_{\nu}=2.984 \pm 0.008$ [99] and provides a bound on $\left|V_{\ell 4}\right|^{2}$ similar to but somewhat weaker than the ones obtained by lepton-universality.

A combined analysis of an old set of unitarity bounds [71], which does not include the one from the CKM matrix determination, leads to the following limits at 90\% C.L. $\left|V_{e 4}\right|^{2}<$ $0.012,\left|V_{\mu 4}\right|^{2}<0.0096$ and $\left|V_{\tau 4}\right|^{2}<0.016$. If the CKM matrix constraint is included and partial cancellations between the contributions of different flavors are taken into account, a previous combined study [70] then gives the more robust limits at 90\% C.L., $\left|V_{e 4}\right|^{2}<$ 0.0066, $\left|V_{\mu 4}\right|^{2}<0.0060$ and $\left|V_{\tau 4}\right|^{2}<0.018$. A very recent analysis $\| 72$ has updated these results using the latest electroweak precision data, except for the CKM observables. They find at $90 \%$ C.L.

$$
\left|V_{e 4}\right|^{2}<0.003, \quad\left|V_{\mu 4}\right|^{2}<0.003, \quad\left|V_{\tau 4}\right|^{2}<0.006 .
$$

If the constraints from CKM observables are included, we expect the bounds to become somewhat stronger, given by $\left|V_{e 4}\right|^{2}<0.002,\left|V_{\mu 4}\right|^{2}<4 \times 10^{-5},\left|V_{\tau 4}\right|^{2}<0.006$ [100]. In 
Table 1: Most stringent model-independent constraints on the mixing elements of the heavy neutrino from precision electro-weak measurements. The bounds on $\left|V_{\ell 4}\right|^{2}, \ell=e, \mu, \tau$ and on $\left|V_{e 4} V_{\mu 4}\right|$ at $90 \%$ C.L.. See text for details.

\begin{tabular}{|c|c|c|}
\hline Mixing element & Range of $m_{4}$ & EW Measurement \\
\hline$\left|V_{e 4}\right|^{2}$ & $m_{4} \gtrsim \mathcal{O}\left(m_{\pi}\right)$ & $<0.003[72]$ \\
\hline$\left|V_{\mu 4}\right|^{2}$ & $m_{4} \gtrsim \mathcal{O}\left(m_{\Lambda}\right)$ & $<0.003[72]$ \\
\hline$\left|V_{\tau 4}\right|^{2}$ & $m_{4} \gtrsim \mathcal{O}\left(m_{\tau}\right)$ & $<0.006[\overline{72}]$ \\
\hline$\left|V_{e 4} V_{\mu 4}\right|$ & $10 \mathrm{GeV}(100 \mathrm{GeV})[1000 \mathrm{GeV}]$ & $<0.015\left(3.5 \times 10^{-4}\right)\left[1.2 \times 10^{-4}\right]$ \\
\hline
\end{tabular}

the following, we take the bound $\left|V_{\mu 4}\right|^{2}<0.0060$ as a conservative reference limit on the mixing for comparison with the results of our study.

Indirect limits on the parameters characterizing heavy sterile neutrinos can be obtained from searches for flavour changing neutral current processes such as $\mu \rightarrow e \gamma, \mu \rightarrow e e^{+} e^{-}$ and $\mu-e$ conversion in nuclei $|75|$. The branching fraction for $\mu \rightarrow e \gamma$ induced by the mixing with heavy singlet neutrinos is given by [73, 74, 75]:

$$
\operatorname{Br}(\mu \rightarrow e \gamma)=\frac{3 \alpha}{8 \pi}\left|\sum_{m^{\prime}} V_{e m^{\prime}} V_{\mu m^{\prime}}^{*} g\left(\frac{m_{N_{m^{\prime}}}^{2}}{m_{W}^{2}}\right)\right|^{2},
$$

where $m^{\prime}$ indicates the heavy sterile neutrinos with mass $m_{N_{m^{\prime}}}$, and $m_{W}$ is the mass of the $W$ boson. The function $g(x)$ is given by

$$
g(x)=\frac{x\left(1-6 x+3 x^{2}+2 x^{3}-6 x^{2} \ln (x)\right)}{2(1-x)^{4}},
$$

where $g(x)$ goes from 0 to 1 as $x$ varies from 0 to infinity. At present, the branching fraction is constrained to be $\operatorname{Br}(\mu \rightarrow e \gamma)<1.2 \times 10^{-11}$ [101] at 90\% C.L. implying that, for one extra sterile neutrino, $\left|V_{e 4} V_{\mu 4}^{*}\right|<0.015\left(3.5 \times 10^{-4}\right)\left[1.2 \times 10^{-4}\right]$ for $m_{4}=10 \mathrm{GeV}(100 \mathrm{GeV})[1000 \mathrm{GeV}]$. Similar constraints are imposed by searches for the processes $\mu-e$ conversion in nuclei and $\mu \rightarrow e e^{+} e^{-}$[75]. The current strongest bound comes from the search for $\mu-e$ conversion in Ti for which the branching ratio with respect to the total nuclear muon capture rate is constrained to be $\operatorname{Br}(\mu \mathrm{Ti} \rightarrow e \mathrm{Ti})<4.3 \times 10^{-12}$ at $90 \%$ C.L. 102]. For one sterile neutrino, this translates into a bound on the following quartic combination of mixing angles $\left.\left|V_{e 4} V_{\mu 4}^{*} \sum_{\ell}\right| V_{\ell 4}\right|^{2} \mid<1.3 \times 10^{-3}\left(100 \mathrm{GeV} / m_{4}\right)^{2}$, which is weaker than the bounds from $\mu \rightarrow e \gamma$ searches but becomes important at very high values of the masses, $m_{4} \gtrsim 10 \mathrm{TeV}$. In the presence of more than one sterile neutrino, partial cancellations between their contributions are possible, with a consequent weakening of the bounds. Future more sensitive searches will further improve these limits.

The most stringent EW precision constraints are compiled in Table 1 and include the bounds reported above from universality tests and lepton flavor changing processes. These bounds are obtained barring cancellations between mixing angles and therefore could be weakened if some parameters are of the same order.

\subsubsection{Neutrinoless Double Beta Decay $(0 \nu \beta \beta)$}

The most well studied among $\Delta L=2$ processes is neutrinoless double beta decay $(0 \nu \beta \beta)$ and the constraints from it deserve special attention. The constraints on $\left|V_{e 4}\right|^{2}$ for a wide 
range of heavy neutrino masses $\left(10 \mathrm{MeV} \leq \mathrm{m}_{4} \leq 100 \mathrm{GeV}\right)$ are shown in Figs. 2 and 3 . For heavy neutrinos with mass, $m_{N_{m^{\prime}}} \gg 1 \mathrm{GeV}$, the bound is 91, 84]

$$
\sum_{m^{\prime}} \frac{\left|V_{e m^{\prime}}\right|^{2}}{m_{N_{m^{\prime}}}}<5 \times 10^{-5} \mathrm{TeV}^{-1} .
$$

The constraint above is very strong and makes it impossible to observe at colliders the like-sign dilepton signature with electrons (see Sec. 价).

\section{Lepton-Number Violating Decays}

The key point for the search of lepton-number violating processes in this paper is to consider the substantial enhancement via resonant neutrino production. One thus needs to evaluate the decay widths of $N_{4}$ to various channels. We consider the decay width of the heavy Majorana neutrino in two regimes: when the mass is much smaller than that of the $W$ boson and when the mass is larger than the mass of the $W$ boson. Based on this, we then compute the $\Delta L=2$ decay branching fractions for $\tau$ lepton and $K, D, D_{s}$ and $B$ mesons.

\subsection{Decay Modes of Heavy Majorana Neutrino}

\subsubsection{Decay Modes of Heavy Majorana Neutrino with mass $m_{4} \ll m_{W}$}

For the $L V$ low energy tau decays and rare meson decays the resonant contribution is from a heavy Majorana neutrino with mass of order $\mathrm{MeV}$ to $\mathrm{GeV}$. In this section we discuss the decay modes of a Majorana neutrino which is lighter than the $W$ boson, so that $m_{4} \ll m_{W}$. The heavy neutrino decays via charged and neutral current interactions to the modes listed below. The partial decay widths of the heavy Majorana neutrino with the leading terms in mixing and in the massless limit of the final state particles are given below. The full detailed expressions for the same are given in Appendix C.

$$
\begin{aligned}
\Gamma^{\ell P} & \equiv \Gamma\left(N_{4} \rightarrow \ell^{-} P^{+}\right)=\frac{G_{F}^{2}}{16 \pi} f_{P}^{2}\left|V_{q \bar{q}^{\prime}}\right|^{2}\left|V_{\ell 4}\right|^{2} m_{4}^{3}, \\
\Gamma^{\nu_{\ell} P} & \equiv \Gamma\left(N_{4} \rightarrow \nu_{\ell} P^{0}\right)=\frac{G_{F}^{2}}{64 \pi} f_{P}^{2}\left|V_{\ell 4}\right|^{2} m_{4}^{3}, \\
\Gamma^{\ell V} & \equiv \Gamma\left(N_{4} \rightarrow \ell^{-} V^{+}\right)=\frac{G_{F}^{2}}{16 \pi} f_{V}^{2}\left|V_{q \bar{q}^{\prime}}\right|^{2}\left|V_{\ell 4}\right|^{2} m_{4}^{3}, \\
\Gamma^{\nu_{\ell} V} & \equiv \Gamma\left(N_{4} \rightarrow \nu_{\ell} V^{0}\right)=\frac{G_{F}^{2}}{2 \pi} \kappa_{V}^{2} f_{V}^{2}\left|V_{\ell 4}\right|^{2} m_{4}^{3}, \\
\Gamma^{\ell_{1} \ell_{2} \nu_{\ell_{2}}} & \equiv \Gamma\left(N_{4} \rightarrow \ell_{1}^{-} \ell_{2}^{+} \nu_{\ell_{2}}\right)=\frac{G_{F}^{2}}{192 \pi^{3}}\left|V_{\ell_{1}}\right|^{2} m_{4}^{5}, \\
\Gamma^{\nu_{\ell_{1}} \ell_{2} \ell_{2}} & \equiv \Gamma\left(N_{4} \rightarrow \nu_{\ell_{1}} \ell_{2}^{-} \ell_{2}^{+}\right)=\frac{G_{F}^{2}}{96 \pi^{3}}\left|V_{\ell_{1} 4}\right|^{2} m_{4}^{5}\left[\alpha_{1}+\delta_{\ell_{1} \ell_{2}} \alpha_{2}\right], \\
\Gamma^{\nu_{\ell_{1}} \nu \nu} & \equiv \sum_{\ell_{2}=e}^{\tau} \Gamma\left(N_{4} \rightarrow \nu_{\ell_{1}} \nu_{\ell_{2}} \overline{\nu_{\ell_{2}}}\right)=\frac{G_{F}^{2}}{96 \pi^{3}}\left|V_{\ell_{1} 4}\right|^{2} m_{4}^{5},
\end{aligned}
$$

where $P^{+(0)}$ and $V^{+(0)}$ are charged (neutral) pseudoscalar and vector mesons, $f_{M}$ are the meson decay constants and $V_{q \bar{q}^{\prime}}$ are the CKM matrix elements. 
All the decay modes listed above contribute to the total decay width of the heavy Majorana neutrino which is given by:

$$
\begin{aligned}
\Gamma_{N_{4}} & =\sum_{\ell, P} \Gamma^{\nu_{\ell} P}+\sum_{\ell, V} \Gamma^{\nu_{\ell} V}+\sum_{\ell, P} 2 \Gamma^{\ell P}+\sum_{\ell, V} 2 \Gamma^{\ell V} \\
& +\sum_{\ell_{1}, \ell_{2}\left(\ell_{1} \neq \ell_{2}\right)} 2 \Gamma^{\ell_{1} \ell_{2} \nu_{\ell_{2}}}+\sum_{\ell_{1}, \ell_{2}} \Gamma^{\nu_{\ell_{1}} \ell_{2} \ell_{2}}+\sum_{\ell_{1}} \Gamma^{\nu_{\ell_{1}} \nu \nu},
\end{aligned}
$$

where $\ell, \ell_{1}, \ell_{2}=e, \mu, \tau$. For a Majorana neutrino, the $\Delta L=0$ process $N_{4} \rightarrow \ell^{-} P^{+}$as well as its charge conjugate $\Delta L=2$ process $N_{4} \rightarrow \ell^{+} P^{-}$are possible and have the same width $\Gamma^{\ell P}$. Hence the factor of 2 associated with the decay width of this mode in Eq. (3.8). Similarly, the $\Delta L=0$ and its charge conjugate $\Delta L=2$ process are possible for the decay modes $N_{4} \rightarrow \ell^{-} V^{+}$and $N_{4} \rightarrow \ell_{1}^{-} \ell_{2}^{+} \nu_{\ell_{2}}$ and hence have a factor of 2 associated with their width in Eq. (3.8).

For the low energy $L V$ tau decays and rare meson decays we consider, the mass of the heavy neutrino is in the range $140 \mathrm{MeV} \lesssim m_{4} \lesssim 5278 \mathrm{MeV}$. For this mass range we list all the possible decay channels for $N_{4}$ in Table 6 in Appendix 9 . The mass and decay constants of pseudoscalar and vector mesons used in the calculation of partial widths given in Eqs. (3.1) - 3.7) are listed in Table 7 in Appendix E.

Next, we get a rough numerical estimate of the total width of the heavy neutrino with the decay modes discussed in Eqs. (3.1) - (3.7). To do this, we consider the massless limit of the decay products of the heavy neutrino, include only leading terms in mixing of $\mathcal{O}\left(\left|V_{\ell 4}\right|^{2}\right)$ and ignore small factors like $\pi$ and $\left|V^{C K M}\right|^{2}$ in calculating the partial decay widths. We can only get a rough estimate of the width in this approximation, but it is sufficient to see that it warrants the use of narrow width approximation. The two body decays of the heavy neutrino have a general form

$$
\Gamma^{2 b o d y} \sim \frac{G_{F}^{2} f_{M}^{2} m_{4}^{3}}{10 \pi}\left|V_{\ell 4}\right|^{2} \sim \frac{G_{F}^{2} f_{M}^{2} m_{4}^{3}}{10}\left|V_{\ell 4}\right|^{2} \sim\left(10^{-13}\left|V_{\ell 4}\right|^{2}\right) \mathrm{GeV}
$$

where typical values of $m_{4} \sim 1 \mathrm{GeV}, f_{M} \sim 0.1 \mathrm{GeV}$ and $G_{F} \sim 10^{-5} \mathrm{GeV}^{-2}$ have been used. The three body decays of the heavy neutrino have a general form

$$
\Gamma^{3 b o d y} \sim \frac{G_{F}^{2} m_{4}^{5}}{100 \pi^{3}}\left|V_{\ell 4}\right|^{2} \sim \frac{G_{F}^{2} m_{4}^{5}}{1000}\left|V_{\ell 4}\right|^{2} \sim\left(10^{-13}\left|V_{\ell 4}\right|^{2}\right) \mathrm{GeV},
$$

where typical values of $m_{4} \sim 1 \mathrm{GeV}$ and $G_{F} \sim 10^{-5} \mathrm{GeV}^{-2}$ have been used. The total width of the heavy neutrino is then given by

$$
\begin{aligned}
\Gamma_{N_{4}} & =\text { (number of decay modes }) \times\left(\Gamma^{2 b o d y}+\Gamma^{3 b o d y}\right) \\
& \sim 50 \times\left(10^{-13} \mathrm{GeV}+10^{-13} \mathrm{GeV}\right)\left|\mathrm{V}_{\ell 4}\right|^{2} \sim\left(10^{-11}\left|\mathrm{~V}_{\ell 4}\right|^{2}\right) \mathrm{GeV}
\end{aligned}
$$

As shown above, the width of the heavy neutrino $\sim \mathcal{O}\left(10^{-11}\left|V_{\ell 4}\right|^{2}\right) \mathrm{GeV}$ is much smaller than the mass of the heavy neutrino $\sim \mathcal{O}(1 \mathrm{GeV})$ and we can use the narrow width approximation to an excellent approximation.

Now we look at the lifetime of the heavy Majorana neutrino to determine the decay length. The lifetime is given by

$$
\begin{aligned}
\tau_{N_{4}} & =\frac{1}{\Gamma_{N_{4}}} \sim \frac{1}{10^{-11}\left|V_{\ell 4}\right|^{2} \mathrm{GeV}}, \\
& \sim 10^{11}\left|V_{\ell 4}\right|^{-2} \mathrm{GeV}^{-1} \sim 6.58 \times 10^{-14}\left|\mathrm{~V}_{\ell 4}\right|^{-2} \mathrm{~s}
\end{aligned}
$$


which gives a typical decay length $c \tau_{N_{4}} \sim 1 \times 10^{-5}\left|V_{\ell 4}\right|^{-2} \mathrm{~m}$. Note that for a very small mixing, $\left|V_{\ell 4}\right|^{2}<\mathcal{O}\left(10^{-5}\right)$, the $N_{4}$ may escape from the detector if it is not much heavier than a $\mathrm{GeV}$. We will take this effect into account in the following studies.

\subsubsection{Decay Modes of Heavy Majorana Neutrino with mass $m_{4}>m_{W}$}

In this section we discuss the decay modes of the Majorana neutrino which is heavier than the $W$ gauge boson, so that $m_{4}>m_{W}$. The decay modes of the heavy Majorana neutrino are to a $W$ or a $Z$ gauge boson plus the corresponding SM lepton. The partial decay widths for longitudinal and transverse gauge bosons $W^{ \pm}, Z^{0}$ in static heavy neutrino frame are

$$
\begin{aligned}
\Gamma^{\ell W_{L}} & \equiv \Gamma\left(N_{4} \rightarrow \ell^{-} W_{L}^{+}\right)=\Gamma\left(N_{4} \rightarrow \ell^{+} W_{L}^{-}\right)=\frac{g^{2}}{64 \pi M_{W}^{2}}\left|V_{\ell 4}\right|^{2} m_{4}^{3}\left(1-\mu_{W}\right)^{2}, \\
\Gamma^{\ell W_{T}} & \equiv \Gamma\left(N_{4} \rightarrow \ell^{-} W_{T}^{+}\right)=\Gamma\left(N_{4} \rightarrow \ell^{+} W_{T}^{-}\right)=\frac{g^{2}}{32 \pi}\left|V_{\ell 4}\right|^{2} m_{4}\left(1-\mu_{W}\right)^{2} \\
\Gamma^{\nu \ell} Z_{L} & \equiv \Gamma\left(N_{4} \rightarrow \nu_{\ell} Z_{L}\right)=\frac{g^{2}}{64 \pi M_{W}^{2}}\left|V_{\ell 4}\right|^{2} m_{4}^{3}\left(1-\mu_{Z}\right)^{2} \\
\Gamma^{\nu} Z_{T} & \equiv \Gamma\left(N_{4} \rightarrow \nu_{\ell} Z_{T}\right)=\frac{g^{2}}{32 \pi \cos _{W}^{2}}\left|V_{\ell 4}\right|^{2} m_{4}\left(1-\mu_{Z}\right)^{2}
\end{aligned}
$$

where $\mu_{i}$ are the masses of the gauge bosons scaled by the mass of the heavy neutrino and are given by $\mu_{i}=m_{i}^{2} / m_{4}^{2}$. To obtain the total decay width for $N_{4}$, we sum over the charged leptons $\ell$ and as discussed earlier include the $\Delta L=0$ process $N_{4} \rightarrow \ell^{-} W_{L, T}^{+}$as well as the charge conjugate $\Delta L=2$ process $N_{4} \rightarrow \ell^{+} W_{L, T}^{-}$. Hence the factor of 2 associated with the decay width of these modes in the expression for the total width below.

$$
\Gamma_{N_{4}}=\sum_{\ell}\left(2 \Gamma^{\ell W_{L}}+2 \Gamma^{\ell W_{T}}+\Gamma^{\nu_{\ell} Z_{L}}+\Gamma^{\nu_{\ell} Z_{T}}\right)
$$

In Eqs. (3.15) - (3.17), we have used the relation (see Appendix for details)

$$
\sum_{m=1}^{3}\left|U_{m 4}^{\nu N}\right|^{2}=\left[\sum_{\ell=e}^{\tau}\left|V_{\ell 4}\right|^{2}\left(1-\sum_{\ell_{1}=e}^{\tau}\left|V_{\ell_{1} 4}\right|^{2}\right)\right], \text { since } U U^{\dagger}+V V^{\dagger}=I .
$$

Ignoring terms of order $\left|V_{\ell 4}\right|^{4}$ we have

$$
\sum_{m}\left|U_{m 4}^{\nu N}\right|^{2} \approx \sum_{\ell}\left|V_{\ell 4}\right|^{2} .
$$

In this approximation, the total width of a heavy Majorana neutrino can be written as

$$
\Gamma_{N_{4}} \begin{cases}\approx \sum_{\ell}\left|V_{\ell 4}\right|^{2} \frac{3 G_{F} m_{4}^{3}}{8 \pi \sqrt{2}} & \text { for } m_{4}>m_{W}, \\ \propto \sum_{\ell}\left|V_{\ell 4}\right|^{2} G_{F}^{2} m_{4}^{3}\left(f_{M}^{2}+m_{4}^{2}\right) & \text { for } m_{4} \ll m_{W},\end{cases}
$$

where the expression when $m_{4} \ll m_{W}$ is obtained from Eq. (3.8) and $f_{M}$ are the meson decay constants. We note that the approximate form of the total width as given in Eq. (3.20) 

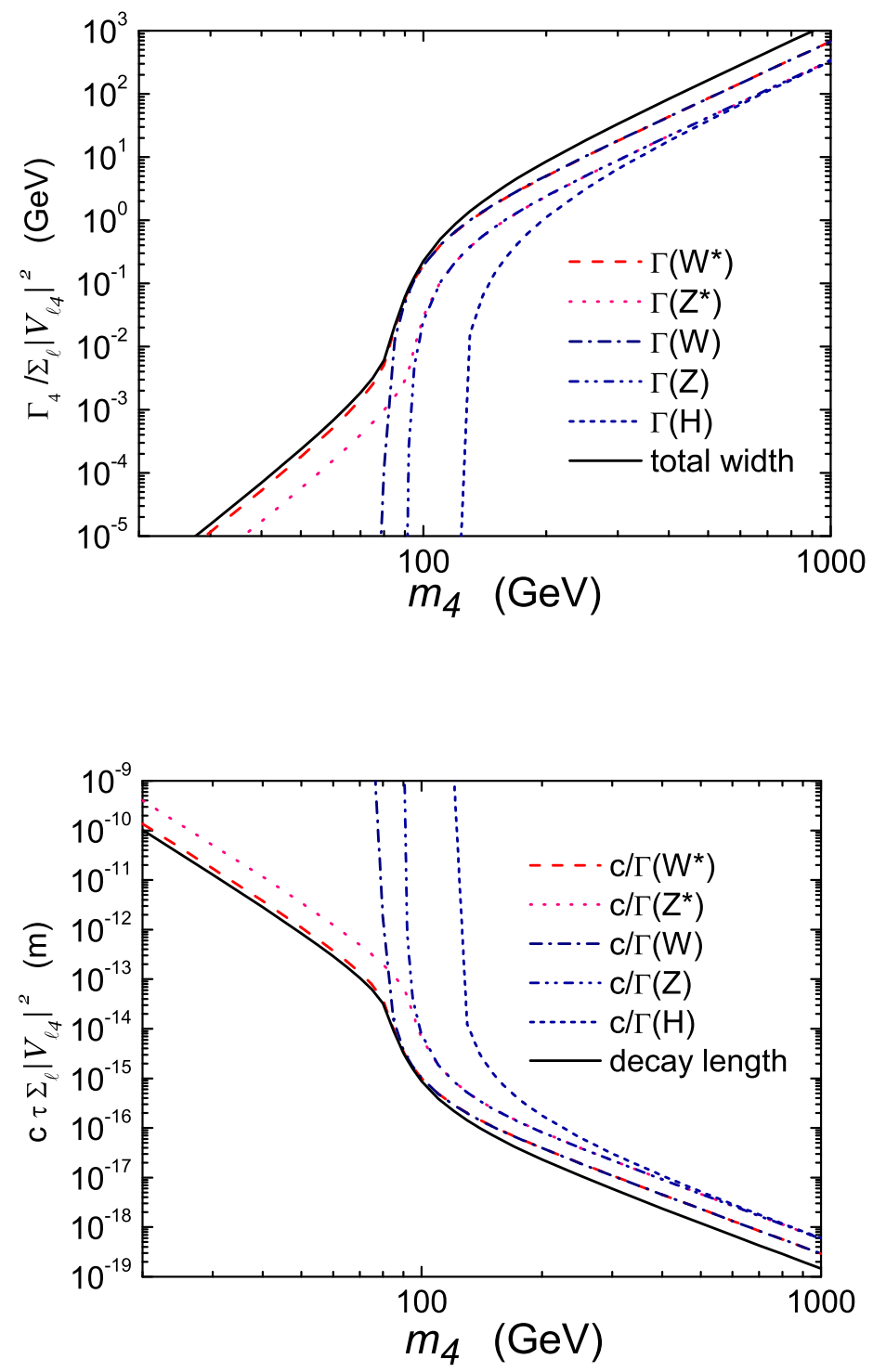

Figure 6: (a) Top: decay width and (b) bottom: decay length (normalized by $\sum_{\ell}\left|V_{\ell 4}\right|^{2}$ ) versus mass of heavy Majorana neutrino for real and virtual weak bosons with the inclusion of Higgs decay channel for $m_{H}=120 \mathrm{GeV}$.

is only for intuitive purposes to infer the general behaviour of the total width as a function of mass. The precise expressions for the total width of the heavy Majorana neutrino as given in Eqs. (3.8), (3.17) and ( $\overline{\text { C.9 }}$ ) have been used in the numerical analysis.

It should be noted that in the SM, if $N_{4}$ is heavier than the Higgs boson, then the decay to a Higgs will be present and the partial width is given by

$$
\Gamma^{\nu H} \equiv \Gamma\left(N_{4} \rightarrow \nu_{\ell} H\right)=\frac{g^{2}}{64 \pi m_{W}^{2}}\left|V_{\ell 4}\right|^{2} m_{4}^{3}\left(1-\mu_{H}\right)^{2} .
$$

In Fig. 6 we plot the decay width of the heavy Majorana neutrino versus its mass normalized 

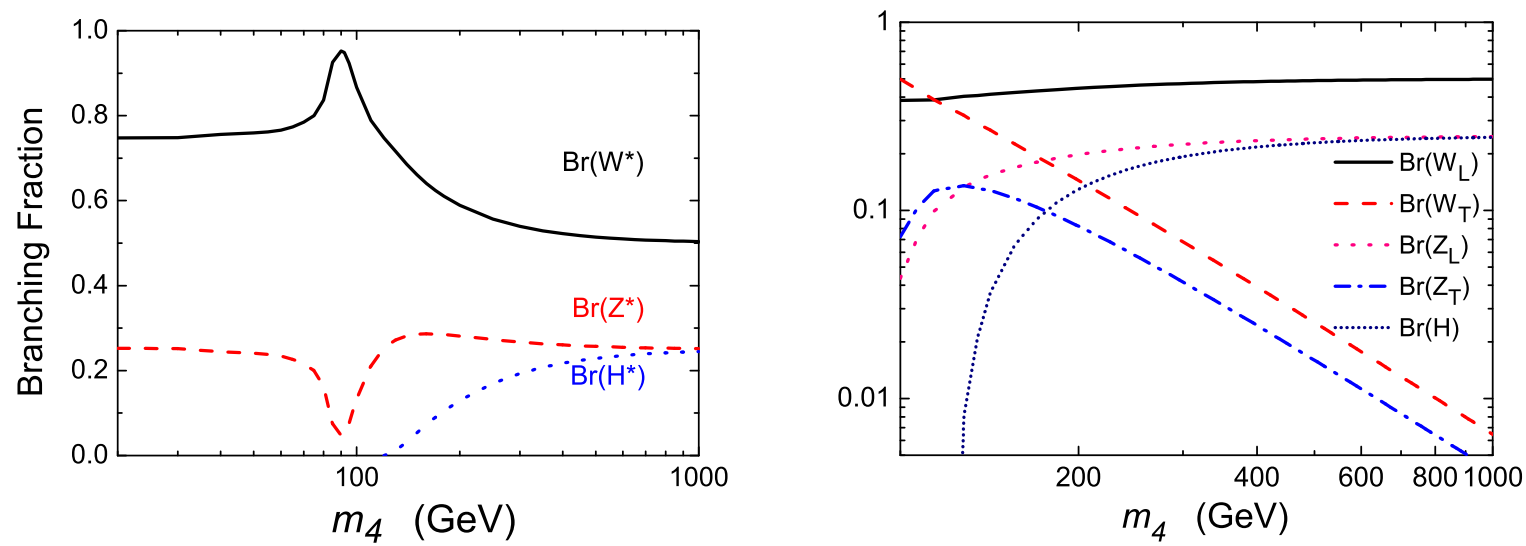

Figure 7: (a) Left: branching fractions for decay of heavy Majorana neutrino into $W^{*}$ and $Z^{*}$ bosons with varying heavy neutrino mass; (b) right: branching fractions for decay of heavy Majorana neutrino into longitudinal and transverse gauge bosons in static heavy neutrino frame with the inclusion of Higgs decay channel for $m_{H}=120 \mathrm{GeV}$.

by the common factor $\sum_{\ell}\left|V_{\ell 4}\right|^{2}$. We can see in Fig. 6(a) that for a heavy neutrino with mass $m_{4}>m_{W}$, the decay width increases as $G_{F} m_{4}^{3}$ as given in Eq. (3.20). Given the rather small mixing parameter, the width remains narrow even for $m_{4} \sim \mathcal{O}(1 \mathrm{TeV})$. For a lighter neutrino with $m_{4} \ll m_{W}$, the width can be very small. The proper decay length is presented in Fig. 6(b). We see from this that for $m_{4} \lesssim 20 \mathrm{GeV}$ and $\left|V_{\mu 4}\right|^{2} \lesssim 10^{-4}$ from Fig. 1 , we have $c \tau \sim 1 \mu \mathrm{m}$.

In Fig. 1(a) we plot the branching fractions of the heavy Majorana neutrino decay to $W \ell$ and $Z \nu$ versus varying heavy neutrino mass $m_{4}$. In Fig. [1(b) we plot the branching fractions for the decays into longitudinal and transverse gauge bosons in static heavy neutrino frame. When the neutrino mass is large, it mainly decays to longitudinal gauge bosons and $\operatorname{Br}\left(N_{4} \rightarrow W^{+} \ell^{-}\right) \simeq \operatorname{Br}\left(N_{4} \rightarrow Z \nu\right)=\operatorname{Br}\left(N_{4} \rightarrow H \nu\right)=25 \%$. In terms of the search at hadron colliders, we prefer to adopt the $W \ell$ mode since we wish to reconstruct the full event including the lepton number.

\subsection{Lepton-Number Violating Tau Decays}

In this section we examine tau decays into an anti-lepton and two mesons

$$
\tau^{-}\left(p_{1}\right) \rightarrow \ell^{+}\left(p_{2}\right) M_{1}^{-}\left(q_{1}\right) M_{2}^{-}\left(q_{2}\right)
$$

which is a process with $\Delta L=-2$. The decay amplitude for the above process is given by

$$
i \mathcal{M}=2 G_{F}^{2} V_{M_{1}}^{C K M} V_{M_{2}}^{C K M} f_{M_{1}} f_{M_{2}} V_{\tau 4}^{*} V_{\ell 4}^{*} m_{4}\left[\frac{\overline{v_{\tau}} q_{1} q_{2} P_{R} v_{\ell}}{\left(p_{1}-q_{1}\right)^{2}-m_{4}^{2}+i \Gamma_{N_{4}} m_{4}}\right]+\left(q_{1} \leftrightarrow q_{2}\right),
$$

where $V_{M_{i}}^{C K M}$ and $f_{M_{i}}$ are the quark flavor mixing element and the decay constant for the meson $M_{i}$ respectively. From this decay amplitude, we can calculate the transition rate $\Gamma_{L V}^{\tau}$ and the branching fraction normalised by the tau decay width. In Appendix $\mathrm{D}$, we give the calculations and the full expressions for the decay branching fraction of the 
Table 2: Mass and mixing elements of heavy neutrino and the decay mode constraining them with the corresponding experimental bounds on branching fractions. Bounds for $\Delta L=2$ tau decays are from Ref. [103]

\begin{tabular}{|c|c|l|l|}
\hline Mixing element & Range of $m_{4}(\mathrm{MeV})$ & Decay mode & $B_{\exp }$ \\
\hline \multirow{3}{*}{$\left|V_{e 4} V_{\tau 4}\right|$} & $140-1637$ & $\tau^{-} \rightarrow e^{+} \pi^{-} \pi^{-}$ & $2.7 \times 10^{-7}$ \\
& $140-1637$ & $\tau^{-} \rightarrow e^{+} \pi^{-} K^{-}$ & $1.8 \times 10^{-7}$ \\
& $494-1283$ & $\tau^{-} \rightarrow e^{+} K^{-} K^{-}$ & $1.5 \times 10^{-7}$ \\
\hline \multirow{3}{*}{$\left|V_{\mu 4} V_{\tau 4}\right|$} & $245-1637$ & $\tau^{-} \rightarrow \mu^{+} \pi^{-} \pi^{-}$ & $0.7 \times 10^{-7}$ \\
& $245-1637$ & $\tau^{-} \rightarrow \mu^{+} \pi^{-} K^{-}$ & $2.2 \times 10^{-7}$ \\
& $599-1283$ & $\tau^{-} \rightarrow \mu^{+} K^{-} K^{-}$ & $4.8 \times 10^{-7}$ \\
\hline
\end{tabular}

process 3.22$)$ in terms of the mass of heavy neutrino, $m_{4}$, and the mixing $\left|V_{\tau 4} V_{\ell 4}\right|^{2}$. To understand the physical picture, we can express the branching fraction in an intuitive form, in the massless limit of the final state particles, as

$$
\begin{aligned}
\mathrm{Br} & =\frac{\Gamma_{L V}^{\tau}}{\Gamma_{\tau}}=\Gamma_{L V}^{\tau}\left(\frac{192 \pi^{3}}{G_{F}^{2} m_{\tau}^{5}}\right), \\
& \sim \frac{3}{2} \pi\left(1-\frac{1}{2} \delta_{M_{1} M_{2}}\right) G_{F}^{2} f_{M_{1}}^{2} f_{M_{2}}^{2}\left|V_{M_{1}}^{C K M} V_{M_{2}}^{C K M}\right|^{2}\left|V_{\tau 4} V_{\ell 4}\right|^{2}\left(1-\frac{m_{4}^{2}}{m_{\tau}^{2}}\right)\left(\frac{m_{4}}{\Gamma_{N_{4}}}\right), \\
& \sim 10^{-3}\left|V_{M_{1}}^{C K M} V_{M_{2}}^{C K M}\right|^{2}\left|V_{\tau 4} V_{\ell 4}\right|,
\end{aligned}
$$

where we have used typical values of $m_{4} \sim 1 \mathrm{GeV}, f_{M_{i}} \sim 0.1 \mathrm{GeV}, G_{F} \sim 1 \times 10^{-5} \mathrm{GeV}^{-2}$ and $\Gamma_{N_{4}} \sim 10^{-11}\left|V_{\ell 4}\right|^{2} \mathrm{GeV}$. From the simple expression given above one can easily make a rough estimate of the required sensitivity and hence the feasibility of observation in terms of the mixing parameters for a given model.

A direct search for $L V$ tau decays has been made at the BaBar detector and the limits on the branching fractions were reported in Ref. 103]. The experimental limits for various decay modes are typically of the order of $10^{-7}$, as given in Table 2. From the nonobservation of the $L V$ tau decay modes one can determine bounds on the mixing parameters $\left|V_{\ell 4} V_{\tau 4}\right|^{2}$ as a function of the heavy neutrino mass $m_{4}$. To do this in a comprehensive manner, we carry out a Monte Carlo sampling of the mixing parameters and the mass of the heavy neutrino. For simplicity, the mixing elements $V_{e 4}, V_{\mu 4}$ and $V_{\tau 4}$ are allowed to vary in the range from 0 to 1 . The ranges of mass sampled for the heavy neutrino are listed in Table 2 for the various tau decay modes. We only sample the range of masses that lead to a resonant enhancement of the width as the other mass regions have very small transition rates as discussed earlier. We then calculate the transition rates and branching fractions over the entire range of mixing and mass of the heavy neutrino and the results of the Monte Carlo sampling are discussed next.

The relevant mixing parameters $\left|V_{e 4} V_{\tau 4}\right|$ and $\left|V_{\mu 4} V_{\tau 4}\right|$ are probed as a function of the heavy neutrino mass $m_{4}$ and are shown in Fig. 8(a) and Fig. 8(b), respectively. Under the assumption that the detector was able to reconstruct all the signal events, the region above the curves is excluded by the current direct experimental search for $L V$ tau decays. The most stringent bound on $\left|V_{e 4} V_{\tau 4}\right|$ is of $\mathcal{O}\left(10^{-6}\right)$ and comes from $\tau^{-} \rightarrow e^{+} \pi^{-} \pi^{-}$. The most stringent bound on $\left|V_{\mu 4} V_{\tau 4}\right|$ is also of $\mathcal{O}\left(10^{-6}\right)$ and comes from $\tau^{-} \rightarrow \mu^{+} \pi^{-} \pi^{-}$. This is three orders of magnitude more sensitive than the limits from precision electroweak data which 
constrain the square of the mixing $\left|V_{\ell 4}\right|^{2}$ to be less than few times $10^{-3}$. In the absence of detection of $L V$ processes the constraints on mixing from peak searches, accelerator experiments, reactor experiments and others (collectively called laboratory constraints here and henceforth) described in Fig. 2 - Fig. 5 are also applicable here. In the mass region probed by $L V$ tau decays the most stringent current constraints are $\left|V_{e 4}\right|^{2}<10^{-7}-10^{-8}$, $\left|V_{\mu 4}\right|^{2}<10^{-6}-10^{-8}$ and $\left|V_{\tau 4}\right|^{2}<10^{-1}-10^{-4}$. This would roughly translate into constraints on $\left|V_{e 4} V_{\tau 4}\right|<10^{-4}-10^{-6}$ and $\left|V_{\mu 4} V_{\tau 4}\right|<10^{-4}-10^{-6}$ which are comparable to the limits from $L V$ tau decay modes. We explore more combinations of mixing elements and also provide better constraints on mixing in some mass regions. To summarize, the constraints on mixing from $L V$ tau decays are always competitive with or better than precision $\mathrm{EW}$ constraints and laboratory constraints in the corresponding mass region. The experimental bounds can improve in future and an order of magnitude improvement in the experimental branching fraction will give approximately an order of magnitude improvement in the constraints for the mixing parameters $\left|V_{\ell 4} V_{\tau 4}\right|$. More importantly, a detection in one of the laboratory experiments implies the existence of a sterile neutrino while a detection in one of the modes studied in our analysis would imply $L V$ and hence the existence of a Majorana neutrino.

It should be noted that the intermediate heavy Majorana neutrino is treated as a real particle which propagates before decaying. If it exits the experimental apparatus prior to decaying, then the signal corresponding to the $\Delta L=2$ process cannot be reconstructed and no bound could be deduced from the non-observation of such events. In Figs. \&(a) and $8(\mathrm{~b})$, we provide an estimate of the bound on the mixing parameters which takes into account the probability of the heavy Majorana neutrino to decay within the detector of size $L_{\text {exp }}$. This probability is given by

$$
P=1-\exp \left(-L_{\exp } \Gamma_{N}\right)
$$

and for small masses and/or small mixing parameters and consequently long decay lengths, it can be approximated with $P \simeq L_{\exp } \Gamma_{N}$. We take $L_{\exp }=10 \mathrm{~m}$, the typical size of the detectors used in the experiments under consideration. For simplicity, we take $N_{4}$ to be relativistic but we keep its gamma factor $\gamma=1$, as a more precise value requires a full understanding of the experimental setup. We assume $\left|V_{e 4}\right|=\left|V_{\mu 4}\right|=\left|V_{\tau 4}\right|$. An estimate of the realistic bound on the mixing parameter $\left|V_{e 4} V_{\tau 4}\right|$ is then given by

$$
\left|V_{e 4} V_{\tau 4}\right|\left(=\left|V_{e 4}\right|^{2}\right)=\sqrt{\left|V_{e 4}\right|_{\infty}^{2} /\left(L_{\exp } \Gamma_{N 0}\right)},
$$

where $\left|V_{e 4}\right|_{\infty}^{2}$ is the bound obtained assuming that all the $N_{4}$ decay in the detector and discussed above, and $\Gamma_{N 0}$ is the decay rate for a fully active heavy Majorana neutrino, i.e. when the mixing parameter $\left|V_{\ell 4}\right|=1$ for $\ell=e, \mu, \tau$. The bounds remain unchanged for large values of the mixing angle and /or large values of $m_{4}$, as the decay length in these cases is very short. However, the most sensitive limit on $\left|V_{e 4} V_{\tau 4}\right|\left(=\left|V_{e 4}\right|^{2}\right)$ coming from $\tau \rightarrow e \pi \pi$ searches gets weakened to $\sim 4 \times 10^{-4}\left(4 \times 10^{-5}\right)\left(1 \times 10^{-5}\right)$ for $m_{4}=0.2(0.5)(1.0) \mathrm{GeV}$. Similarly, the searches for $\tau \rightarrow \mu \pi \pi$ allows to set a bound on $\left|V_{\mu 4} V_{\tau 4}\right|\left(=\left|V_{\mu 4}\right|^{2}\right)$ which weakens to $\left|V_{\mu 4} V_{\tau 4}\right|<1 \times 10^{-4}\left(2 \times 10^{-5}\right)\left(1 \times 10^{-5}\right)$ for $m_{4}=300$ (600) (900) MeV. A detailed analysis taking into account the experimental setup should be performed in order to obtain more precise bounds. 

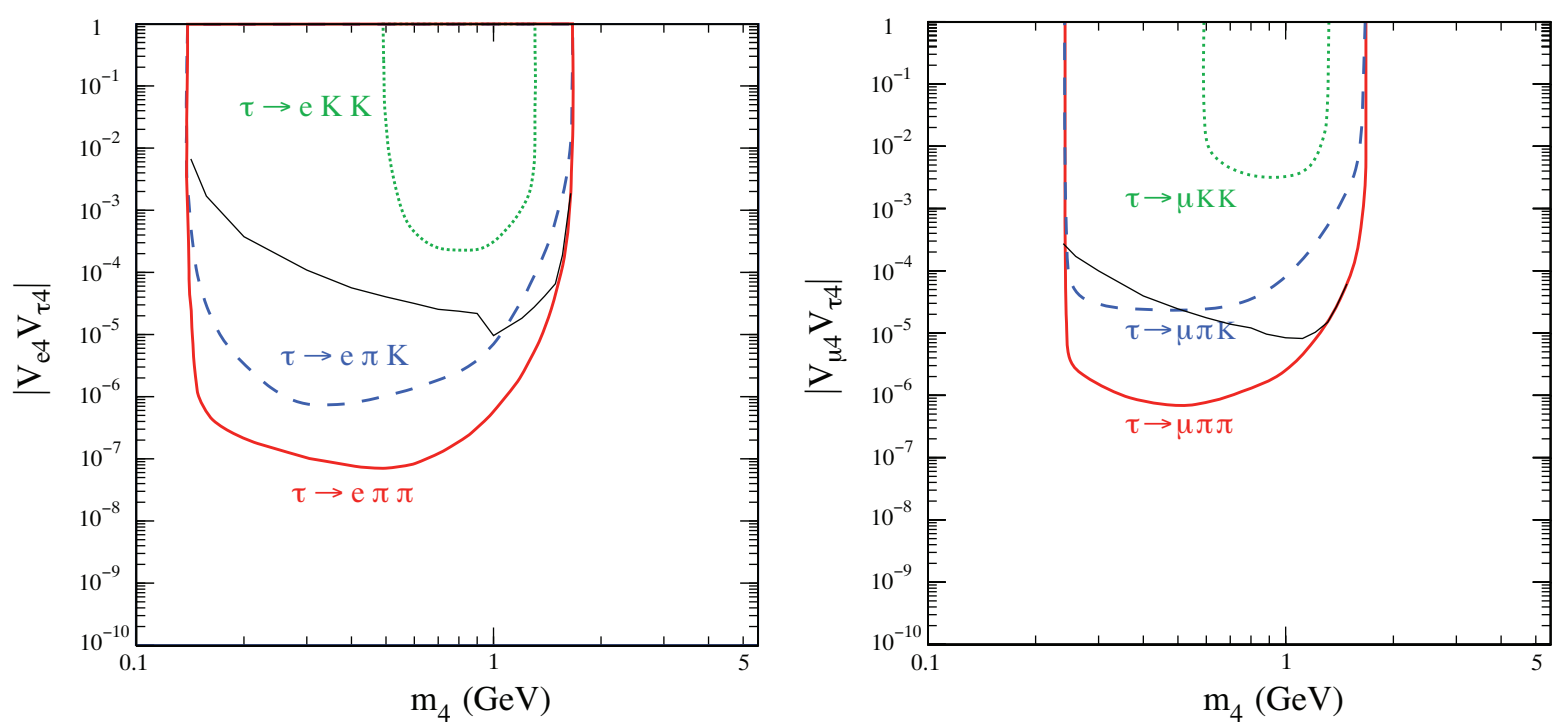

Figure 8: (a) Left: excluded regions above the curves for $\left|V_{e 4} V_{\tau 4}\right|$ versus $m_{4}$; (b) right: same as (a) but for $\left|V_{\mu 4} V_{\tau 4}\right|$. The thin black lines correspond to the estimate of the bound (for $\tau \rightarrow e \pi \pi$ and $\tau \rightarrow \mu \pi \pi$ ) once the probability of $N_{4}$ decay in the detector is taken into account.

\subsection{Lepton-Number Violating Rare Meson Decays}

We now investigate the $L V$ processes in which a meson decays into two like-sign leptons and another meson

$$
M_{1}^{+}\left(q_{1}\right) \rightarrow \ell^{+}\left(p_{1}\right) \ell^{+}\left(p_{2}\right) M_{2}^{-}\left(q_{2}\right) .
$$

These decays are similar to the tau decay modes described in the previous section. The decay amplitude for the above process is given by

$$
\begin{aligned}
i \mathcal{M}^{P} & =2 G_{F}^{2} V_{M_{1}}^{C K M} V_{M_{2}}^{C K M} f_{M_{1}} f_{M_{2}} V_{\ell_{1} 4} V_{\ell_{2} 4} m_{4} \\
& \times\left[\frac{\overline{u_{\ell_{1}}} q_{1} q_{2} P_{R} v_{\ell_{2}}}{\left(q_{1}-p_{1}\right)^{2}-m_{4}^{2}+i \Gamma_{N_{4}} m_{4}}\right]+\left(p_{1} \leftrightarrow p_{2}\right), \\
i \mathcal{M}^{V} & =2 G_{F}^{2} V_{M_{1}}^{C K M} V_{M_{2}}^{C K M} f_{M_{1}} f_{M_{2}} V_{\ell_{1} 4} V_{\ell_{2} 4} m_{4} m_{M_{2}} \\
& \times\left[\frac{\overline{u_{\ell_{1}}} q_{1} \not k^{\lambda}\left(q_{2}\right) P_{R} v_{\ell_{2}}}{\left(q_{1}-p_{1}\right)^{2}-m_{4}{ }^{2}+i \Gamma_{N_{4}} m_{4}}\right]+\left(p_{1} \leftrightarrow p_{2}\right),
\end{aligned}
$$

where $i \mathcal{M}^{P}$ and $i \mathcal{M}^{V}$ are the decay amplitudes when the meson $M_{2}$ is a pseudoscalar or vector meson respectively and $V_{M_{i}}^{C K M}$ and $f_{M_{i}}$ are the quark flavor mixing element and the decay constant for the meson $M_{i}$ respectively. From this decay amplitude, we can calculate the transition rate $\Gamma_{L V}^{M_{1}}$ and the branching fraction normalised by the decay width of the meson $M_{1}$. In Appendix $\mathbb{E}$, we give the calculations for the decay branching fraction of the process (3.27) in terms of the mass of heavy neutrino, $m_{4}$, and the mixing $\left|V_{\ell_{1} 4} V_{\ell_{2} 4}\right|$. We can express the branching fraction in an intuitive form, in the massless limit of the final state particles, as

$$
\mathrm{Br}=\frac{\Gamma_{L V}^{M_{1}}}{\Gamma_{M_{1}}}=\Gamma_{L V}^{M_{1}} \tau_{M_{1}},
$$


Table 3: Same as Table 2 but for $\Delta L=2$ rare meson decays. The experimental bounds are from Ref. 99], the bounds for $D^{+} \rightarrow e^{+} e^{+} \pi^{-}\left(K^{-}\right)$are from Ref. [104.

\begin{tabular}{|c|c|c|c|}
\hline Mixing element & Range of $m_{4}(\mathrm{MeV})$ & Decay mode & $B_{\exp }$ \\
\hline \multirow{9}{*}{$\left|V_{e 4}\right|^{2}$} & $140-493$ & $K^{+} \rightarrow e^{+} e^{+} \pi^{-}$ & $6.4 \times 10^{-10}$ \\
\hline & $140-1868$ & $D^{+} \rightarrow e+e^{+} \pi^{-}$ & $3.6 \times 10^{-6}$ \\
\hline & $494-1868$ & $D^{+} \rightarrow e^{+} e^{+} K^{-}$ & $4.5 \times 10^{-6}$ \\
\hline & $140-1967$ & $D_{s}^{+} \rightarrow e^{+} e^{+} \pi^{-}$ & $6.9 \times 10^{-4}$ \\
\hline & $494-1967$ & $D_{s}^{+} \rightarrow e^{+} e^{+} K^{-}$ & $6.3 \times 10^{-4}$ \\
\hline & $140-5278$ & $B^{+} \rightarrow e^{+} e^{+} \pi^{-}$ & $1.6 \times 10^{-6}$ \\
\hline & $494-5278$ & $B^{+} \rightarrow e^{+} e^{+} K^{-}$ & $1.0 \times 10^{-6}$ \\
\hline & $776-5278$ & $B^{+} \rightarrow e^{+} e^{+} \rho^{-}$ & $2.6 \times 10^{-6}$ \\
\hline & $892-5278$ & $B^{+} \rightarrow e^{+} e^{+} K^{*-}$ & $2.8 \times 10^{-6}$ \\
\hline \multirow{12}{*}{$\left|V_{\mu 4}\right|^{2}$} & $245-388$ & $K^{+} \rightarrow \mu^{+} \mu^{+} \pi^{-}$ & $3.0 \times 10^{-9}$ \\
\hline & $245-1763$ & $D^{+} \rightarrow \mu^{+} \mu^{+} \pi^{-}$ & $4.8 \times 10^{-6}$ \\
\hline & $599-1763$ & $D^{+} \rightarrow \mu^{+} \mu^{+} K^{-}$ & $1.3 \times 10^{-5}$ \\
\hline & $881-1763$ & $D^{+} \rightarrow \mu^{+} \mu^{+} \rho^{-}$ & $5.6 \times 10^{-4}$ \\
\hline & $997-1763$ & $D^{+} \rightarrow \mu^{+} \mu^{+} K^{*-}$ & $8.5 \times 10^{-4}$ \\
\hline & $245-1862$ & $D_{s}^{+} \rightarrow \mu^{+} \mu^{+} \pi^{-}$ & $2.9 \times 10^{-5}$ \\
\hline & $599-1862$ & $D_{s}^{+} \rightarrow \mu^{+} \mu^{+} K^{-}$ & $1.3 \times 10^{-5}$ \\
\hline & $997-1862$ & $D_{s}^{+} \rightarrow \mu^{+} \mu^{+} K^{*-}$ & $1.4 \times 10^{-3}$ \\
\hline & $245-5173$ & $B^{+} \rightarrow \mu^{+} \mu^{+} \pi^{-}$ & $1.4 \times 10^{-6}$ \\
\hline & $599-5173$ & $B^{+} \rightarrow \mu^{+} \mu^{+} K^{-}$ & $1.8 \times 10^{-6}$ \\
\hline & $881-5173$ & $B^{+} \rightarrow \mu^{+} \mu^{+} \rho^{-}$ & $5.0 \times 10^{-6}$ \\
\hline & $997-5173$ & $B^{+} \rightarrow \mu^{+} \mu^{+} K^{*-}$ & $8.3 \times 10^{-6}$ \\
\hline \multirow{9}{*}{$\left|V_{e 4} V_{\mu 4}\right|$} & $140-493$ & $K^{+} \rightarrow e^{+} \mu^{+} \pi^{-}$ & $5.5 \times 10^{-10}$ \\
\hline & $140-1868$ & $D^{+} \rightarrow e^{+} \mu^{+} \pi^{-}$ & $5.0 \times 10^{-5}$ \\
\hline & $494-1868$ & $D^{+} \rightarrow e^{+} \mu^{+} K^{-}$ & $1.3 \times 10^{-4}$ \\
\hline & $140-1862$ & $D_{s}^{+} \rightarrow e^{+} \mu^{+} \pi^{-}$ & $7.3 \times 10^{-4}$ \\
\hline & $494-1967$ & $D_{s}^{+} \rightarrow e^{+} \mu^{+} K^{-}$ & $6.8 \times 10^{-4}$ \\
\hline & $140-5278$ & $B^{+} \rightarrow e^{+} \mu^{+} \pi^{-}$ & $1.3 \times 10^{-6}$ \\
\hline & $494-5278$ & $B^{+} \rightarrow e^{+} \mu^{+} K^{-}$ & $2.0 \times 10^{-6}$ \\
\hline & $776-5278$ & $B^{+} \rightarrow e^{+} \mu^{+} \rho^{-}$ & $3.3 \times 10^{-6}$ \\
\hline & $892-5278$ & $B^{+} \rightarrow e^{+} \mu^{+} K^{*-}$ & $4.4 \times 10^{-6}$ \\
\hline
\end{tabular}

$$
\begin{aligned}
& \sim \frac{1}{64 \pi^{2}}\left(1-\frac{1}{2} \delta_{\ell_{1} \ell_{2}}\right) G_{F}^{4} f_{M_{1}}^{2} f_{M_{2}}^{2}\left|V_{M_{1}}^{C K M} V_{M_{2}}^{C K M}\right|^{2}\left|V_{\ell_{1} 4} V_{\ell_{2} 4}\right|^{2}\left(1-\frac{m_{4}^{2}}{m_{\tau}^{2}}\right) m_{M_{1}}^{5} \tau_{M_{1}}\left(\frac{m_{4}}{\Gamma_{N_{4}}}\right), \\
& \sim\left(10^{-16} \mathrm{GeV}\right) \tau_{\mathrm{M}_{1}}\left|\mathrm{~V}_{\mathrm{M}_{1}}^{\mathrm{CKM}} \mathrm{V}_{\mathrm{M}_{2}}^{\mathrm{CKM}}\right|^{2}\left|\mathrm{~V}_{\ell_{1} 4} \mathrm{~V}_{\ell_{2} 4}\right|,
\end{aligned}
$$

where we have used typical values of $m_{4} \sim 1 \mathrm{GeV}, f_{M_{i}} \sim 0.1 \mathrm{GeV}, G_{F} \sim 1 \times 10^{-5} \mathrm{GeV}^{-2}$, $\Gamma_{N_{4}} \sim 10^{-11}\left|V_{\ell 4}\right|^{2} \mathrm{GeV}$ and $\tau_{M_{1}}$ is in seconds. Using the values for the lifetimes of the mesons in Appendix $\mathbb{E}$, the branching fractions for the various mesons are given by

$$
\begin{aligned}
\operatorname{Br}(K) & \sim\left|V_{M_{1}}^{C K M} V_{M_{2}}^{C K M}\right|^{2}\left|V_{\ell_{1} 4} V_{\ell_{2} 4}\right|, \\
\operatorname{Br}(D, B) & \sim 10^{-4}\left|V_{M_{1}}^{C K M} V_{M_{2}}^{C K M}\right|^{2}\left|V_{\ell_{1}} V_{\ell_{2} 4}\right|, \\
\operatorname{Br}\left(D_{s}\right) & \sim 10^{-5}\left|V_{M_{1}}^{C K M} V_{M_{2}}^{C K M}\right|^{2}\left|V_{\ell_{1} 4} V_{\ell_{2} 4}\right| .
\end{aligned}
$$


As mentioned earlier, with the simple expressions above one can easily make a rough estimate of the required sensitivity and hence the feasibility of observation in terms of the mixing parameters for a given model.

Searches for rare meson decay modes have been made in numerous experiments. Table 3 summarizes the current experimental limits on branching fractions given by Refs. 99, 104]. From the non-observation of these $L V$ rare meson decay modes one can determine constraints on mixing parameters $\left|V_{\ell_{1} 4} V_{\ell_{2}}\right|$ as a function of the heavy neutrino mass $m_{4}$. To do this in a comprehensive manner, we carry out a Monte Carlo sampling of the mixing parameters and the mass of the heavy neutrino similar to tau decay. The mixing elements $V_{e 4}, V_{\mu 4}$ and $V_{\tau 4}$ are allowed to range from 0 to 1 for simplicity. Only the range of mass that leads to a resonant enhancement of the width is sampled for the heavy neutrino and listed in Table 3 for the various meson decay modes. The transition rates and branching fractions are then calculated over the entire range of mixing and mass of the heavy neutrino and the results of the Monte Carlo sampling are discussed next.

For the various decay modes, the mixing parameters probed are $\left|V_{e 4}\right|^{2},\left|V_{e 4} V_{\mu 4}\right|$ and $\left|V_{\mu 4}\right|^{2}$ depending on the final state leptons. Again, we plot the excluded region of the mixing parameters as a function of neutrino mass, as shown in Fig. 9 for $\left|V_{e 4}\right|^{2}$, Fig. 10 for $\left|V_{e 4} V_{\mu 4}\right|$ and in Fig. 11 for $\left|V_{\mu 4}\right|^{2}$. The regions above the curves are excluded by the current direct experimental searches for $L V$ meson decays. First we plot the limits which can be derived assuming that all $N_{4}$ decay in the detector and give a positive signature. The most stringent constraints are from the $K^{+} \rightarrow \ell_{1}^{+} \ell_{2}^{+} \pi^{-}$mode with mixings of $\mathcal{O}\left(10^{-9}\right)$ excluded for $\left|V_{e 4}\right|^{2},\left|V_{e 4} V_{\mu 4}\right|$ and $\left|V_{\mu 4}\right|^{2}$. This is six orders of magnitude more sensitive than the limits from precision electroweak data which constrains the square of the mixing $\left|V_{\ell 4}\right|^{2}$ to be less than few times $10^{-3}$. Next in sensitivity are the $D$ and $D_{s}$ decay modes with constraints of order few times $10^{-3}$ which are similar to the constraints from precision electroweak data. The bounds for the same mixing elements are much weaker in the mass range above $2 \mathrm{GeV}$. Even though the limits are weak in this region, it is important not to neglect the experimental study of these processes. It only implies that there is a large parameter space available for the mass and mixing of heavy neutrinos.

As discussed for the $\Delta L=2$ tau decays, in the absence of detection of $L V$ processes the laboratory constraints on mixing described in Fig. 2 - Fig. 5 are also applicable here. In the mass region probed by $L V$ meson decays the most stringent laboratory bounds are $\left|V_{e 4}\right|^{2}<10^{-7}-10^{-8},\left|V_{\mu 4}\right|^{2}<10^{-6}-10^{-8}$ for $m_{4}<2 \mathrm{GeV}$ and $\left|V_{\mu 4}\right|^{2}<10^{-4}$ for $m_{4}>2 \mathrm{GeV}$. This would roughly translate into constraints on $\left|V_{e 4} V_{\mu 4}\right|<10^{-6}-10^{-8}$ for $m_{4}<2 \mathrm{GeV}$ and $\left|V_{e 4} V_{\mu 4}\right|<10^{-5}-10^{-6}$ for $m_{4}>2 \mathrm{GeV}$. It should be noted that, if these experiments were able to fully reconstruct the signal, the limits from $K$ meson decays would be better than the laboratory constraints by at least an order of magnitude in the corresponding mass region. In fact, the constraints on $\left|V_{e 4}\right|^{2}$ from the kaon decay mode $K^{+} \rightarrow e^{+} e^{+} \pi^{-}$would be more stringent than even the constraints from $0 \nu \beta \beta$ shown in Fig. 3. Usually $0 \nu \beta \beta$ experiments have the best sensitivity as they have an advantage of a large "effective luminosity" resulting from the large number of nuclei available for decay. The meson (and tau) experiments on the other hand have a small luminosity coming from a limited number of mesons (taus) produced in accelerators compared to the number of nuclei in $0 \nu \beta \beta$ experiments. It is interesting to note that the resonant enhancement in the case of the $K$ meson decay is able to match or improve over the large "effective luminosity" of $0 \nu \beta \beta$ experiments. In conclusion, the constraints on mixing from $L V$ meson decays are competitive with the precision EW constraints and all the laboratory 


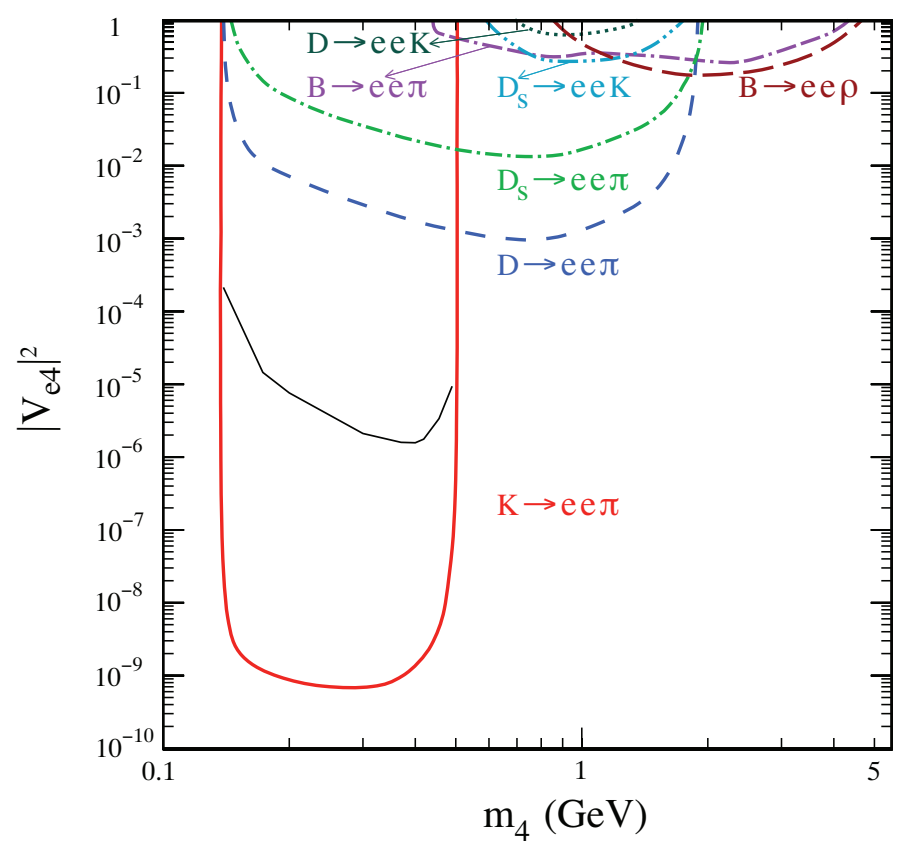

Figure 9: Excluded regions above the curves for $\left|V_{e 4}\right|^{2}$ versus $m_{4}$ from $M_{1}^{+} \rightarrow e^{+} e^{+} M_{2}^{-}$searches. The thin black line corresponds to an estimate of the bound from $K^{+} \rightarrow e^{+} e^{+} \pi^{-}$once the probability of decay of $N_{4}$ in the detector is taken into account.

constraints, potentially even $0 \nu \beta \beta$, in some mass regions. But again, we emphasize that the aim of our analysis is to study $L V$ processes and hence Majorana neutrinos.

We have also taken into account the fact that, for small mixing, only part of the heavy sterile neutrinos produced will decay in the detector. We have considered $L_{\exp }=10 \mathrm{~m}$, $\left|V_{e 4}\right|=\left|V_{\mu 4}\right|=\left|V_{\tau 4}\right|$ and the gamma factor of $N_{4}, \gamma=1$, for simplicity. In this case, as discussed for the $\Delta L=2$ tau decays, the bounds get sensibly weakened. An estimate of these bounds is reported in Figs. 9, 10 and 11 by thin black lines. We see that the bounds get significantly weakened by few orders of magnitude for $K \rightarrow e e \pi, K \rightarrow e \mu \pi$ and $K \rightarrow \mu \mu \pi$ and a careful analysis of these searches should be performed to find the detailed bounds on the mixing angles.

The sensitivity of current direct experimental searches are not adequate to constrain mixings for some decay modes. The theoretically allowed branching fraction versus mass $m_{4}$ for such modes is given in Fig. 12. As we can deduce from Table 3 and Fig. 12 all the modes are very close to start being probed by direct experimental searches. The experimental bounds on branching fractions can improve in future and similar to tau decay modes, an order of magnitude improvement in the experimental branching fraction will give approximately an order of magnitude improvement in the constraints for the mixing parameters $\left|V_{\ell_{1} 4} V_{\ell_{2} 4}\right|$. Currently we do not have any constraints on the mixing parameter $\left|V_{\tau 4}\right|^{2}$ from $L V$ rare meson decay modes. Only very weak constraints for $\operatorname{BR}\left(B \rightarrow X \tau^{+} \tau^{-}\right)<\mathcal{O}(5 \%)$ exist in a theoretical analysis [105]. The similar signature $B^{+} \rightarrow \tau^{+} \tau^{+} M^{-}$is a possible decay mode that would bound $\left|V_{\tau 4}\right|^{2}$ and should be pursued. 


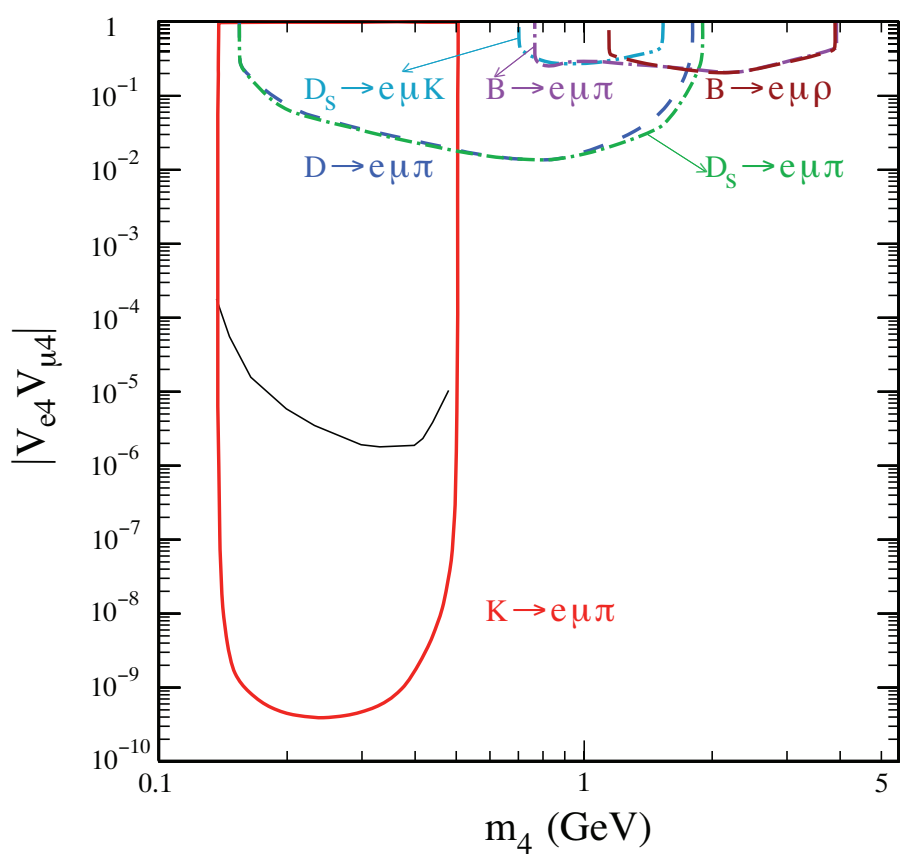

Figure 10: Same as Fig. 9 but for $\left|V_{e 4} V_{\mu 4}\right|$ from $M_{1}^{+} \rightarrow e^{+} \mu^{+} M_{2}^{-}$searches.

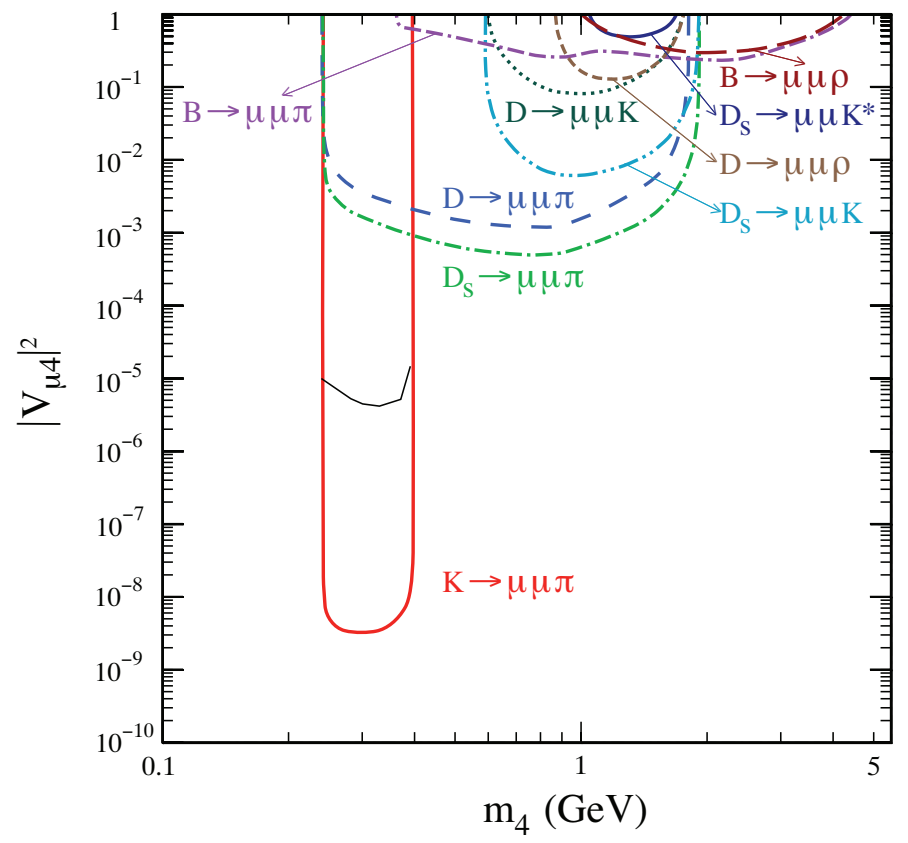

Figure 11: Same as Fig. 9 but for $\left|V_{\mu 4}\right|^{2}$ from $M_{1}^{+} \rightarrow \mu^{+} \mu^{+} M_{2}^{-}$searches.

\section{Collider Signatures}

In this section we study heavy Majorana neutrinos at hadron colliders. The most distinctive channels of the signal involve like-sign di-leptons. It was first proposed in Ref. [29] in the context of the left-right symmetric model, and subsequently studied in Ref. [30, 31, 32, 33] 


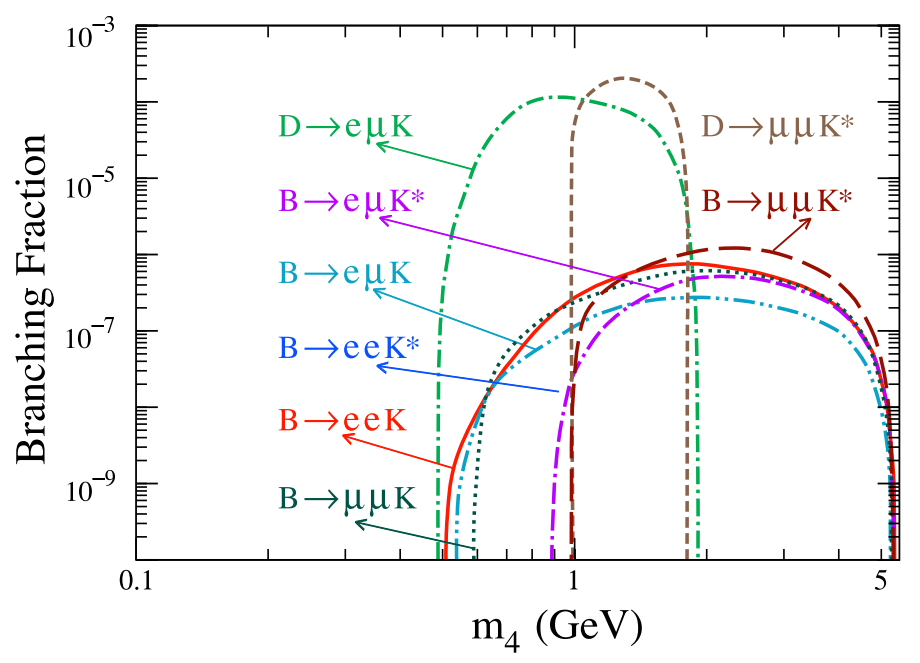

Figure 12: Branching fraction versus heavy neutrino mass $m_{4}$ for decay modes $M_{1}^{+} \rightarrow \ell_{1}^{+} \ell_{2}^{+} M_{2}^{-}$ not yet constrained by direct experimental searches. The regions below the curve are theoretically allowed.

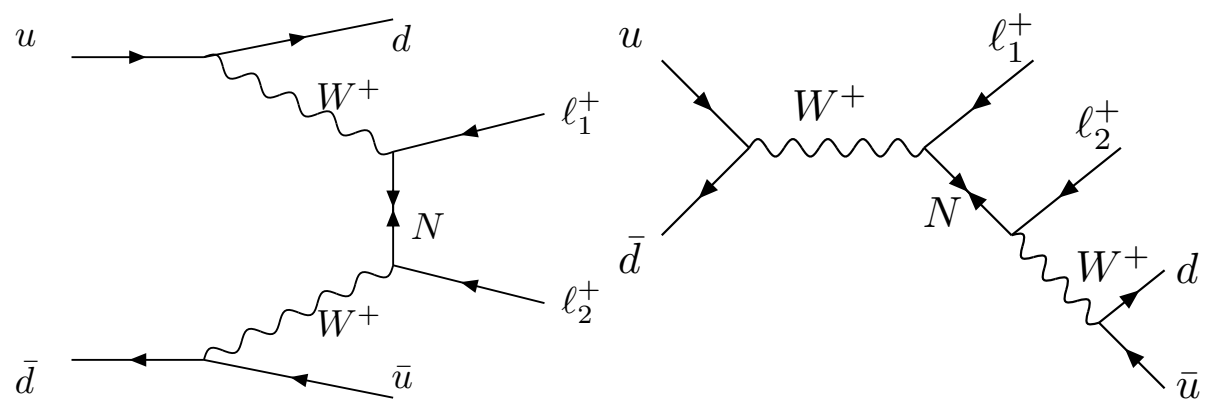

Figure 13: (a) Left: Feynman diagram for like-sign dilepton signature via $W W$ fusion in hadronic collisions; (b) right: the exchanged coherent diagram which is same as heavy neutrino production and decay.

We discuss the signatures for a heavy Majorana neutrino and the sensitivity to probe the parameters $m_{4}$ and $V_{\ell 4}$ at the Tevatron and the LHC.

As for the production of a heavy Majorana neutrino at hadron colliders, the representative diagrams at the parton level are depicted in Fig. 13, with the exchange of final state leptons implied. The first diagram is via $W W$ fusion with a $t$-channel heavy neutrino $N_{4}$ exchange, directly analogous to the process of $0 \nu \beta \beta$. The second diagram is via $s$-channel $N_{4}$ production and subsequent decay. Although in our full calculations, we have coherently counted for all the contributing diagrams of like-sign dilepton production including possible identical particle crossing, it is informative to separately discuss these two classes of diagrams due to their characteristically different kinematics.

The scattering amplitude for the process in Fig. 13(a) is proportional to $V_{\ell_{1} 4} V_{\ell_{2} 4}$ and the cross section can be expressed as

$$
\sigma\left(p p \rightarrow W^{ \pm} W^{ \pm} \rightarrow \ell_{1}^{ \pm} \ell_{2}^{ \pm} X\right)=\left(2-\delta_{\ell_{1} \ell_{2}}\right)\left|V_{\ell_{1} 4} V_{\ell_{2} 4}\right|^{2} \sigma_{0}(W W),
$$

where $\sigma_{0}(W W)$ is the "bare cross section", independent of the mixing parameters. We show the bare cross section at the LHC energy of $14 \mathrm{TeV}$ versus the heavy neutrino mass 


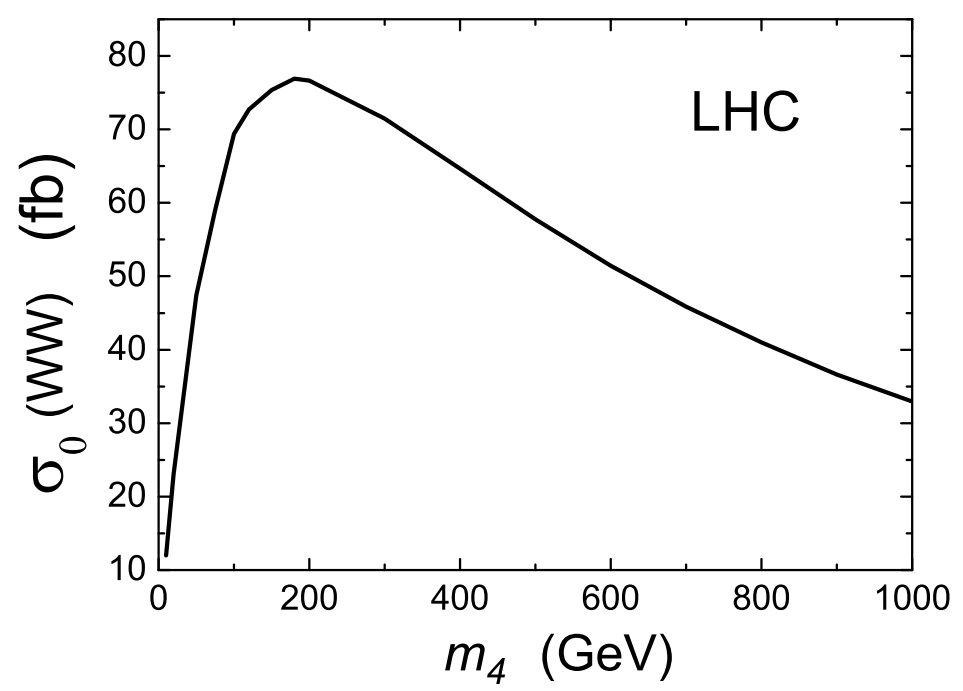

Figure 14: The bare cross section $\sigma_{0}(W W)$ versus mass of the heavy neutrino $m_{4}$.

in Fig. 14. This cross section can be at the order of tens of femtobarns. However, due to the large suppression of the small flavor mixing to the fourth power, the cross section is rather small. This process was calculated in Ref. [20] under the effective vector boson approximation. The authors of Ref. [20] obtained significantly more optimistic results than ours. Further scrutiny indicated that they missed a factor of $G_{F}^{2} m_{W}^{4} / 8$ and their result should be scaled down by this factor. The corresponding curve for the Tevatron is not shown in Fig. 14 as the bare cross section is smaller by nearly two orders of magnitude. Including the small mixing element (to the fourth power) further reduces the cross section drastically with no hope of detection at the Tevatron via this mode.

By far, the dominant production process of heavy Majorana neutrino in hadronic collisions is the diagram shown in Fig. 13(b). We calculate the exact process, but it turns out to be an excellent approximation to parameterize the cross section as

$$
\sigma\left(p p \rightarrow \ell_{1}^{ \pm} \ell_{2}^{ \pm} W^{\mp}\right) \approx\left(2-\delta_{\ell_{1} \ell_{2}}\right) \sigma\left(p p \rightarrow \ell_{1}^{ \pm} N_{4}\right) \operatorname{Br}\left(N_{4} \rightarrow \ell_{2}^{ \pm} W^{\mp}\right) \propto \frac{\left|V_{\ell_{1} 4} V_{\ell_{2}}\right|^{2}}{\sum_{\ell=e}^{\tau}\left|V_{\ell 4}\right|^{2}}
$$

This observation allows us to study the process in a model-independent way. We can rewrite the cross section in a factorized form

$$
\sigma\left(p p \rightarrow \ell_{1}^{ \pm} \ell_{2}^{ \pm} W^{\mp} \rightarrow \ell_{1}^{ \pm} \ell_{2}^{ \pm} j j^{\prime}\right)=\left(2-\delta_{\ell_{1} \ell_{2}}\right) S_{\ell_{1} \ell_{2}} \sigma_{0}\left(N_{4}\right),
$$

where $\sigma_{0}\left(N_{4}\right)$, called the "bare cross section", is only dependent on the mass of heavy neutrino and is independent of all the mixing parameters when the heavy neutrino decay width is narrow. As seen in Fig. 6, this is indeed the case for $m_{4} \lesssim 1 \mathrm{TeV}$ once we fold in the constraints $\left|V_{\ell 4}\right|^{2}<\mathcal{O}\left(10^{-3}\right)$ from precision EW measurements. We calculate the exact cross section for the dilepton production and use the definition Eq. (4.3) to find the bare cross sections $\sigma_{0}\left(N_{4}\right)$, which are shown in Fig. 15 at the Tevatron and the LHC energies 


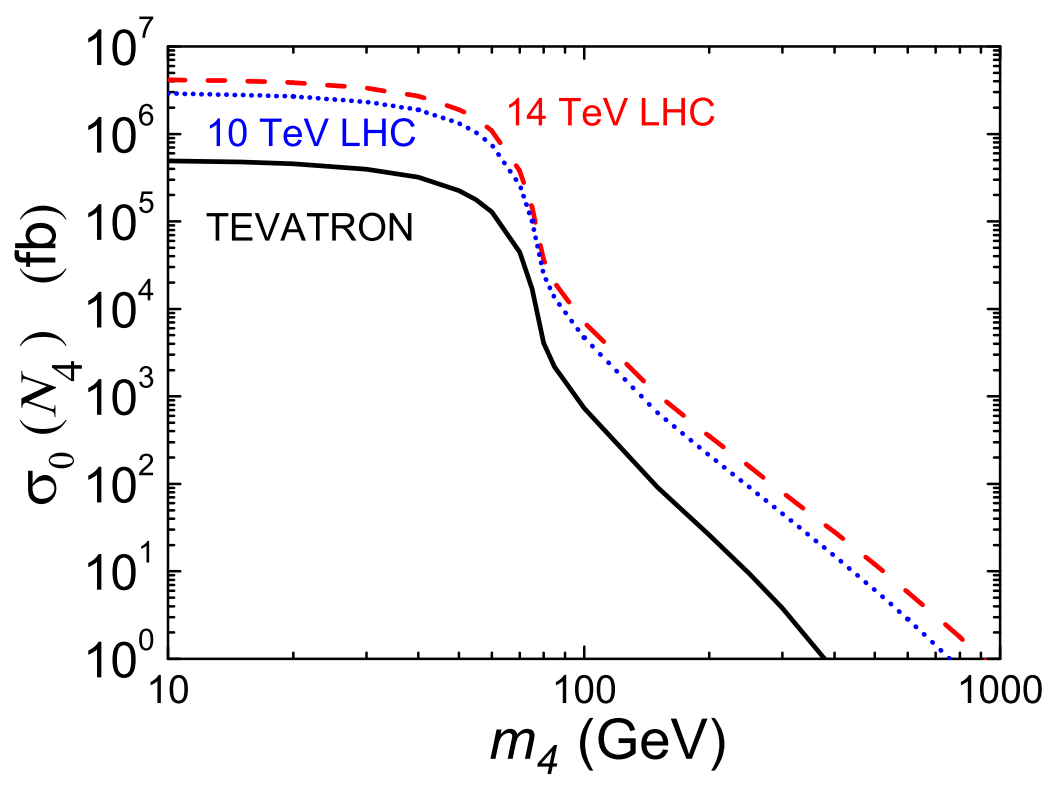

Figure 15: The bare cross section $\sigma_{0}\left(N_{4}\right)$ versus mass of heavy Majorana neutrino $m_{4}$ for the Tevatron ( $p \bar{p}$ at $1.96 \mathrm{TeV}$, solid curve) and the LHC ( $p p$ at 10 and $14 \mathrm{TeV}$, dotted and dashed curves, respectively).

versus the mass of the heavy Majorana neutrino. Due to the fact that the LHC will start its operation at $10 \mathrm{TeV}$, we have calculated the cross sections at both 10 and $14 \mathrm{TeV}$ c.m. energy. The production rate is increased at the higher energy by a factor of 1.5, 2.0, 2.5 for $m_{4}=100,550$ and $1000 \mathrm{GeV}$, respectively. We will mainly present our results at 14 $\mathrm{TeV}$ for the rest of the paper. An obvious feature of the cross sections is the transition near the $W$ mass. For $m_{4}<M_{W}$ the cross section is nearly a constant due to an on-shell $W$ production via the Drell-Yan mechanism with its subsequent leptonic decay to $\ell^{ \pm} N_{4}$. For $m_{4}>M_{W}$ the cross section falls off sharply versus $m_{4}$ and the on-shell decay goes like $N_{4} \rightarrow \ell^{ \pm} W^{\mp} \rightarrow \ell^{ \pm} j_{1} j_{2}$.

The flavor information of the final state leptons is parameterized by

$$
S_{\ell_{1} \ell_{2}}=\frac{\left|V_{\ell_{1} 4} V_{\ell_{2}}\right|^{2}}{\sum_{\ell=e}^{\tau}\left|V_{\ell 4}\right|^{2}},
$$

In general the two final state charged leptons can be of any flavor combination, namely,

$$
e^{ \pm} e^{ \pm}, \quad e^{ \pm} \mu^{ \pm}, \quad e^{ \pm} \tau^{ \pm}, \quad \mu^{ \pm} \mu^{ \pm}, \quad \mu^{ \pm} \tau^{ \pm} \text {and } \tau^{ \pm} \tau^{ \pm}
$$

The constraint from $0 \nu \beta \beta$ as given in Eq. (2.18) is very strong and makes it difficult to observe like-sign di-electrons $e^{ \pm} e^{ \pm}$. The events with $\tau$ leptons will be challenging to reconstruct experimentally. We will thus concentrate on clean dilepton channels of $\mu^{ \pm} \mu^{ \pm}$and $\mu^{ \pm} e^{ \pm}$, although we will comment on our proposal to include the $\tau$ modes. The correspond- 
ing mixing parameters in our notation will be

$$
S_{\mu \mu}=\frac{\left|V_{\mu 4}\right|^{4}}{\sum_{\ell=e}^{\tau}\left|V_{\ell 4}\right|^{2}}, \quad S_{e \mu}=\frac{\left|V_{e 4} V_{\mu 4}\right|^{2}}{\sum_{\ell=e}^{\tau}\left|V_{\ell 4}\right|^{2}},
$$

respectively. Given the smallness of $\left|V_{e 4}\right|^{2}$, we can further simplify our study by exploring only two cases: an optimistic case $\left|V_{\mu 4}\right|^{2} \gg\left|V_{\tau 4}\right|^{2},\left|V_{e 4}\right|^{2}$ and a generic case $\left|V_{\mu 4}\right|^{2} \approx$ $\left|V_{\tau 4}\right|^{2} \gg\left|V_{e 4}\right|^{2}$, which lead to

$$
S_{\mu \mu}=\left\{\begin{array}{cc}
\left|V_{\mu 4}\right|^{2} & \text { (optimistic) } \\
\frac{1}{2}\left|V_{\mu 4}\right|^{2} & \text { (generic) }
\end{array}, \quad S_{\mu e}=\left\{\begin{array}{cc}
\left|V_{e 4}\right|^{2} & \text { (optimistic) } \\
\frac{1}{2}\left|V_{e 4}\right|^{2} & \text { (generic) }
\end{array} .\right.\right.
$$

\subsection{Search for Like-sign Dilepton Signals at the Tevatron}

We now consider the search for $N_{4}$ at the Fermilab Tevatron, which is currently running at a c.m. energy of $1.96 \mathrm{TeV}$ in $p \bar{p}$ collisions. We concentrate on the clean like-sign $\mu^{ \pm} \mu^{ \pm}$ mode

$$
p \bar{p} \rightarrow \mu^{ \pm} \mu^{ \pm} j_{1} j_{2} X,
$$

where $X$ is some inclusive hadronic activities common in hadronic collisions. To quantify the signal observability, we first impose the basic acceptance cuts on leptons and jets to simulate the $\mathrm{CDF} / \mathrm{D} 0$ detector coverage

$$
p_{T}^{\mu}>5 \mathrm{GeV}, \quad\left|\eta^{\mu}\right|<2.0, \quad p_{T}^{j}>10 \mathrm{GeV}, \quad\left|\eta^{j}\right|<3.0 .
$$

We also smear the lepton momentum by a tracking resolution and the jet energy by hadronic calorimeter resolution as

$$
\frac{\Delta p_{T}^{\mu}}{p_{T}^{\mu}}=1.5 \times 10^{-3} p_{T}^{\mu}, \quad \frac{\Delta E_{j}}{E_{j}}=\frac{75 \%}{\sqrt{E_{j}}} \oplus 3 \%,
$$

where $p_{T}^{\mu}$ and $E_{j}$ are in units of $\mathrm{GeV}$.

The signal events we are searching for have very unique kinematical features. For the purpose of illustration, we choose $m_{4}=20,50 \mathrm{GeV}$ (below $m_{W}$ threshold) and $100 \mathrm{GeV}$ (above $m_{W}$ ). First of all, there are two well-isolated like-sign charged leptons. This is shown in Fig. 16(a) by a normalized distribution of the minimal isolation $\Delta R_{\ell j}=\sqrt{\Delta \eta^{2}+\Delta \phi^{2}}$. Second, there is essentially no missing transverse energy. However, realistically, the detectors have finite resolutions as simulated by the Gaussian smearing given in Eq. (4.10). Consequently, there is always some misbalance in the energy-momentum measurements, which is attributed to the missing transverse energies and is plotted in Fig. 16(b). Thirdly, due to the existence of an on-shell $W^{ \pm}$in the signal process, one would expect to reconstruct it by an invariant mass either from the $2 \ell 2 j$ system $m(\ell \ell j j$ ) (in the case of DY production) or from the di-jets $m_{(j j)}$ (in the case of $N_{4}$ decay). This is demonstrated in Figs. 16(c) and (d), respectively. The above kinematical features motivate us to impose the following event selection cuts

$$
\begin{aligned}
& \Delta R_{\ell j}^{\min }>0.5 \\
& 60 \mathrm{GeV}<\text { either } m(\ell \ell j j) \text { or } m(j j)<100 \mathrm{GeV}, \\
& \not p_{T}<20 \mathrm{GeV} .
\end{aligned}
$$



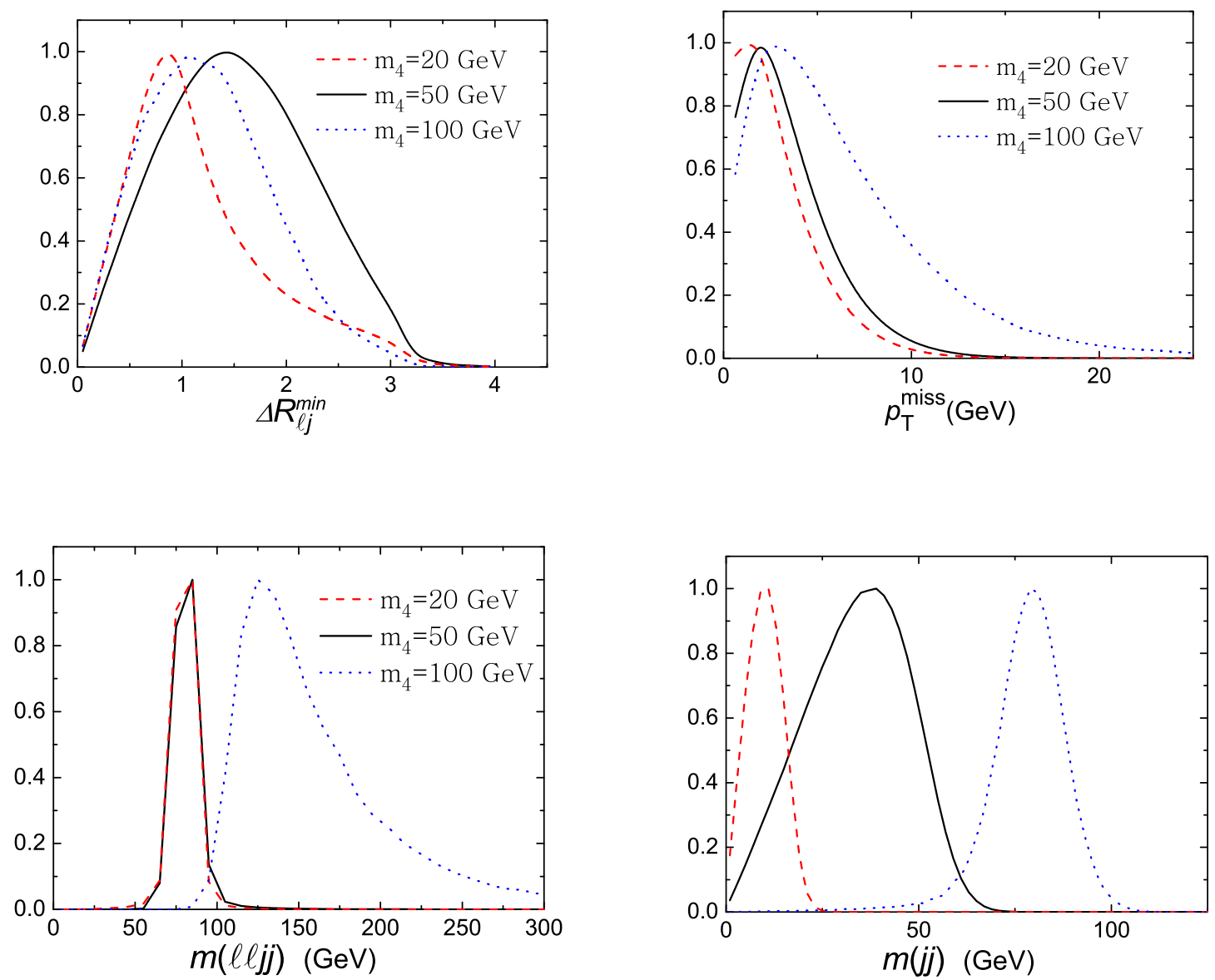

Figure 16: Normalized distributions $\sigma^{-1} d \sigma / d X$ for $m_{4}=20,50$ and $100 \mathrm{GeV}$ at the Tevatron for (a) upper left: the minimal isolation $\Delta R_{\ell j}^{\text {min }}$; (b) upper right: the missing transverse momentum $\not p_{T}$; (c) bottom left: the $2 \ell 2 j$ system invariant mass $m(\ell \ell j j)$; (d) bottom right: the di-jet invariant mass $m(j j)$.

These cuts are highly efficient in selecting the signal events. We illustrate this in Table 4, in which we calculate the signal rates with the consecutive cuts for $m_{4}=60 \mathrm{GeV}$ and $\left|V_{\mu 4}\right|^{2}=\left|V_{\tau 4}\right|^{2}=5 \times 10^{-3} \gg\left|V_{e 4}\right|^{2}$. Note that the choice of mixing elements used in the illustration is motivated by constraints from precision EW measurements. However this is for illustration purposes only and in our full analysis we have kept $S_{\mu \mu}$ as a free parameter.

At the Tevatron energies, the SM contribution to the like-sign dilepton events is rather small. The leading background of this type comes from the top-quark production and its cascade decay via the chain

$$
\begin{aligned}
& t \rightarrow W^{+} b \rightarrow \ell^{+} \nu_{\ell} b, \\
& \bar{t} \rightarrow W^{-} \bar{b} \rightarrow W^{-} \bar{c} \nu_{\ell} \ell^{+} .
\end{aligned}
$$

The background rates and survival probabilities with the consecutive cuts are also given in Table 4 . We see that the $t \bar{t}$ background is essentially eliminated by the selective 
Table 4: The representative signal and background cross sections at the Tevatron, for $\mu^{ \pm} \mu^{ \pm} j j$ and the efficiencies with the consecutive cuts. For illustration, we have used $m_{4}=60 \mathrm{GeV},\left|V_{\mu 4}\right|^{2}=$ $\left|V_{\tau 4}\right|^{2}=5 \times 10^{-3} \gg\left|V_{e 4}\right|^{2}$.

\begin{tabular}{c|c|c|c|c|c}
\hline & No cut & Basic cut (4.9) & $+\Delta R(4.11)$ & $+m(j j), m(\ell \ell j j)(4.12)$ & $+p_{T}(4.13)$ \\
\hline Signal & & & & & \\
$\sigma(\mathrm{fb})$ & 319 & 108 & 99 & 96 & 96 \\
eff. & - & $33 \%$ & $92 \%$ & $97 \%$ & $100 \%$ \\
\hline$t \bar{t} \mathrm{Bkg}$ & & & & & \\
$\sigma(\mathrm{fb})$ & 78.4 & 58.2 & 1.85 & 0.04 & 0.005 \\
eff. & - & $74 \%$ & $3.2 \%$ & $2.2 \%$ & $12.5 \%$ \\
\hline
\end{tabular}

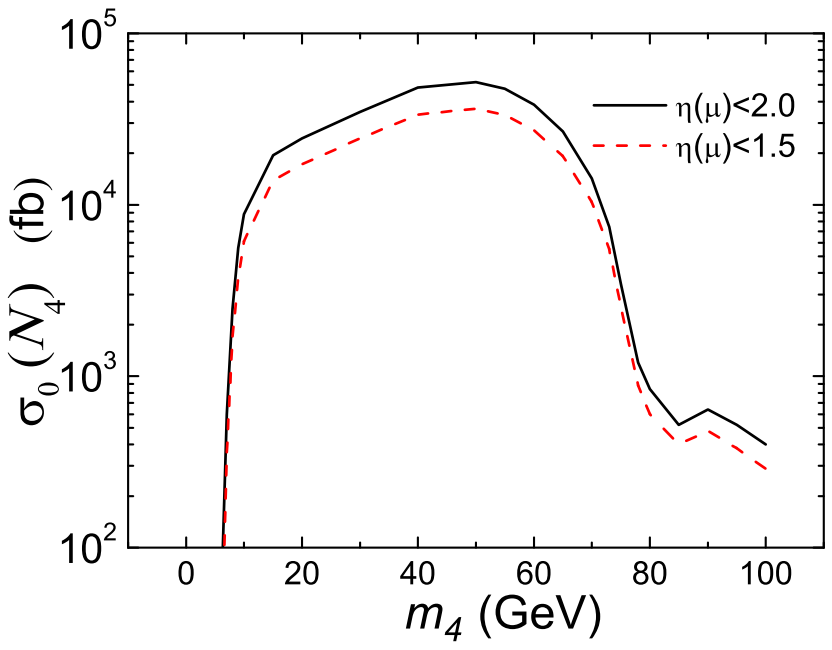

Figure 17: $\sigma_{0}\left(N_{4}\right)$ with varying heavy neutrino mass $m_{4}$ after all the cuts. The two cases correspond to muon rapidity acceptance at D0 and CDF.

cuts. We have also considered other SM backgrounds coming from the production of $W^{ \pm} W^{ \pm} j j, W^{ \pm} Z j j$. After the selective cuts, all these backgrounds are negligibly small.

In Fig. 17, we plot the bare cross section $\sigma_{0}\left(N_{4}\right)$ with the basic cuts of Eq. (4.9) as well as the selection cuts Eqs. (4.11) - 4.13). The reduction in rate is mainly due to the basic acceptance cuts. For comparison, we have also included two choices of pseudo-rapidity cut $|\eta(\mu)|<2$ and $|\eta(\mu)|<1.5$. We now consider the statistical significance of the signal observation. In the absence of background events, we use Poisson statistics to determine the search sensitivity. We take a signal with $95 \%$ Confidence Level (as this is very close to $2 \sigma$ we call it a $2 \sigma$ effect henceforth) to be 3 events. We can thus translate this to the sensitivity to the mixing parameter

$$
\left(2-\delta_{\ell_{1} \ell_{2}}\right) \sigma_{0}\left(N_{4}\right) S_{\ell_{1} \ell_{2}} L \geq 3
$$

where $L$ is the integrated luminosity.

The CDF collaboration at the Tevatron has successfully studied the events with likesign dileptons in a different context [106]. Given our event selection, in Fig. 18 we estimate 
the sensitivity reach for the mixing parameters versus $m_{4}$ at the $2 \sigma$ (solid curves) and $5 \sigma$ (dashed curves) level at the Tevatron. In Figs. 18(a-b) (upper-left and upper-right), the sensitivity is shown for $S_{\mu \mu}$ with 2 and $8 \mathrm{fb}^{-1}$ integrated luminosity. The horizontal dotted lines are the constraint on $S_{\mu \mu} \simeq\left|V_{\mu 4}\right|^{2}<6 \times 10^{-3}$ from an analysis of precision EW measurements [70]. The DELPHI [89] and L3 [90] bounds are also given for comparison. We find that the Tevatron has the potential to reach the following sensitivity for the mass of the heavy neutrino

$$
m_{4} \sim\left\{\begin{array}{c}
40-130 \mathrm{GeV} \text { for } 2 \sigma \text { with } 2 \mathrm{fb}^{-1} \\
10-180 \mathrm{GeV}(50-120 \mathrm{GeV}) \text { for } 2 \sigma(5 \sigma) \text { with } 8 \mathrm{fb}^{-1} .
\end{array}\right.
$$

Alternatively, the sensitivity for the mixing parameter can be

$$
S_{\mu \mu} \sim\left\{\begin{array}{c}
2 \times 10^{-5} \quad \text { for } 2 \sigma \text { with } 2 \mathrm{fb}^{-1} \\
5 \times 10^{-6}\left(2 \times 10^{-5}\right) \text { for } 2 \sigma(5 \sigma) \text { with } 8 \mathrm{fb}^{-1} .
\end{array}\right.
$$

Similar to Figs. 18(a-b), Figs. 18(c-d) (lower-left and lower-right) show the results for $S_{e \mu}$ instead. The lower dotted curve in Fig. 18(d) is the bound on $S_{e \mu} \simeq\left|V_{e 4}\right|^{2}$ from $0 \nu \beta \beta$. We have assumed the same detection efficiencies for $\mu$ and $e$. With this assumption, the slightly better reach for $S_{e \mu}$ compared to $S_{\mu \mu}$ is due the factor of two difference in total rate with identical and nonidentical particles as evident from Eq. (4.3). With $2 \mathrm{fb}^{-1}$ luminosity, the sensitivity to $\left|V_{e 4}\right|^{2}$ is not close to the stringent bound from the $0 \nu \beta \beta$ decay as seen in Fig. 3. We see from Fig. 18(d) that with $8 \mathrm{fb}^{-1}$ luminosity, the Tevatron sensitivity for $S_{e \mu}$ may reach the level of the current bound from $0 \nu \beta \beta$. From Eq. (4.16), it is straightforward to obtain future sensitivity to mixing parameters $\left(S_{\mu \mu}, S_{e \mu}\right)$ by a simple scaling of the luminosity.

\subsection{Search for Like-sign Dilepton Signals at the LHC}

At the LHC with a c.m. energy of $14 \mathrm{TeV}$ in $p p$ collisions, we adopt the basic acceptance cuts on leptons and jets as

$$
p_{T}^{\ell}>10 \mathrm{GeV}, \quad\left|\eta^{\ell}\right|<2.5, \quad p_{T}^{j}>15 \mathrm{GeV}, \quad\left|\eta^{j}\right|<2.5 .
$$

The efficiency of these cuts increases with heavy neutrino mass and is $50 \%$ for $m_{4}=$ $200 \mathrm{GeV}$ and $80 \%$ for $m_{4}=800 \mathrm{GeV}$. The smearing parameters to simulate the ATLAS/CMS detectors are 107.

$$
\frac{\Delta p_{T}^{\mu}}{p_{T}^{\mu}}=36 \times 10^{-5} p_{T}^{\mu}, \quad \frac{\Delta E_{j}}{E_{j}}=\frac{1}{\sqrt{E_{j}}} \oplus 5 \%,
$$

where $p_{T}^{\mu}$ and $E_{j}$ are in units of $\mathrm{GeV}$.

We again present the characteristic kinematical distributions for the signal. Fig. 19.(a) shows the normalized distribution of the minimal isolation $\Delta R_{\ell j}$. The simulated missing transverse momentum after the energy-momentum smearing is plotted in Fig. 19(b). The invariant masses of the $2 \ell 2 j$ system $m(\ell \ell j j)$ and the di-jets $\left.m_{(j j} j\right)$ are demonstrated in Figs. 19(c) and (d), respectively. We thus design the selection cuts at the LHC as

$$
\begin{aligned}
& \Delta R_{\ell j}^{\min }>0.5, \\
& 60 \mathrm{GeV}<\text { either } m(\ell \ell j j) \text { or } m(j j)<100 \mathrm{GeV}, \\
& \not p_{T}<25 \mathrm{GeV} .
\end{aligned}
$$



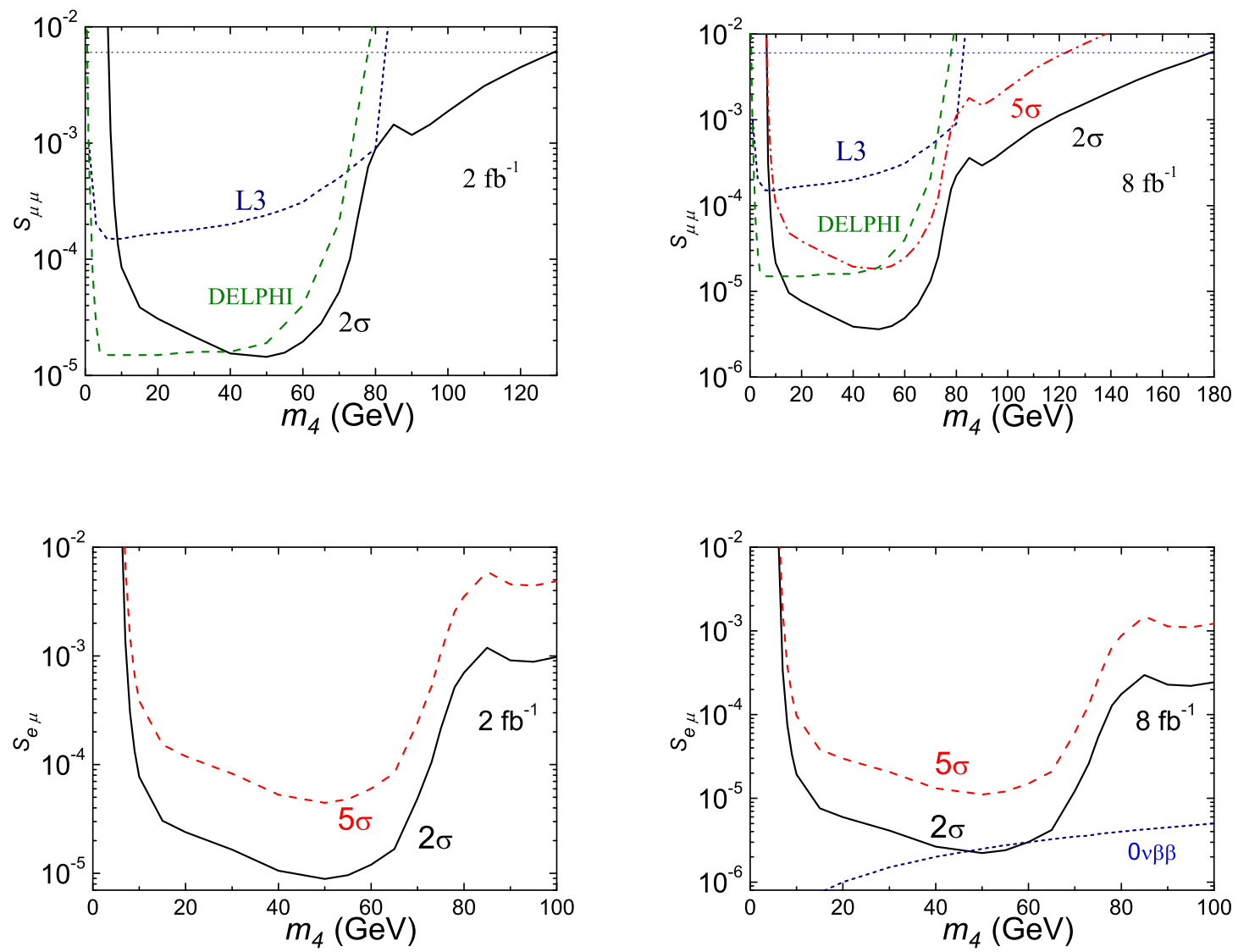

Figure 18: The Tevatron sensitivity to the mixing parameters versus $m_{4}$ (a) upper-left: $2 \sigma$ and $5 \sigma$ sensitivity of $S_{\mu \mu}$ with $2 \mathrm{fb}^{-1}$ integrated luminosity; (b) upper-right: same as (a) but with $8 \mathrm{fb}^{-1}$ integrated luminosity; (c) lower-left: $2 \sigma$ and $5 \sigma$ sensitivity of $S_{e \mu}$ with $2 \mathrm{fb}^{-1}$ integrated luminosity; (d) lower-right: same as (c) but with $8 \mathrm{fb}^{-1}$ integrated luminosity. The horizontal dotted lines in (a) and (b) are the constraint on $S_{\mu \mu} \simeq\left|V_{\mu 4}\right|^{2}<6 \times 10^{-3}$ from an analysis of precision EW measurements [70]. The DELPHI [89 and L3 [90] bounds are also given here for comparison. The lower dotted curve in (d) is the bound on $S_{e \mu} \simeq\left|V_{e 4}\right|^{2}$ from $0 \nu \beta \beta$.

These cuts are highly efficient in selecting the signal events. We illustrate this in Table 5, in which we calculate the signal rates with the consecutive cuts for $m_{4}=200 \mathrm{GeV}$ and $\left|V_{\mu 4}\right|^{2}=\left|V_{\tau 4}\right|^{2}=5 \times 10^{-3} \gg\left|V_{e 4}\right|^{2}$. Again the choice of mixing elements is motivated by constraints from precision EW measurements. However as discussed earlier this is for illustration purposes only and in our full analysis we have kept $S_{\mu \mu}$ and $S_{\mu e}$ as free parameters.

In Fig. 20, we plot the bare cross section $\sigma_{0}\left(N_{4}\right)$ with the basic cuts of Eq. (4.19) as well as the selection cuts Eqs. (4.21) - (4.23) at $14 \mathrm{TeV}$. The solid (dotted) curves correspond to the bare cross section without (with) the Higgs decay channel for $m_{H}=120 \mathrm{GeV}$. The reduction in rate is mainly due to the basic acceptance cuts. We note that the cross section with the cuts at $14 \mathrm{TeV}$ is higher than that at $10 \mathrm{TeV}$ by a factor of $1.4-1.6$ for $m_{4}=100-500 \mathrm{GeV}$. The sensitivity reach for the mixing parameters to be presented later 

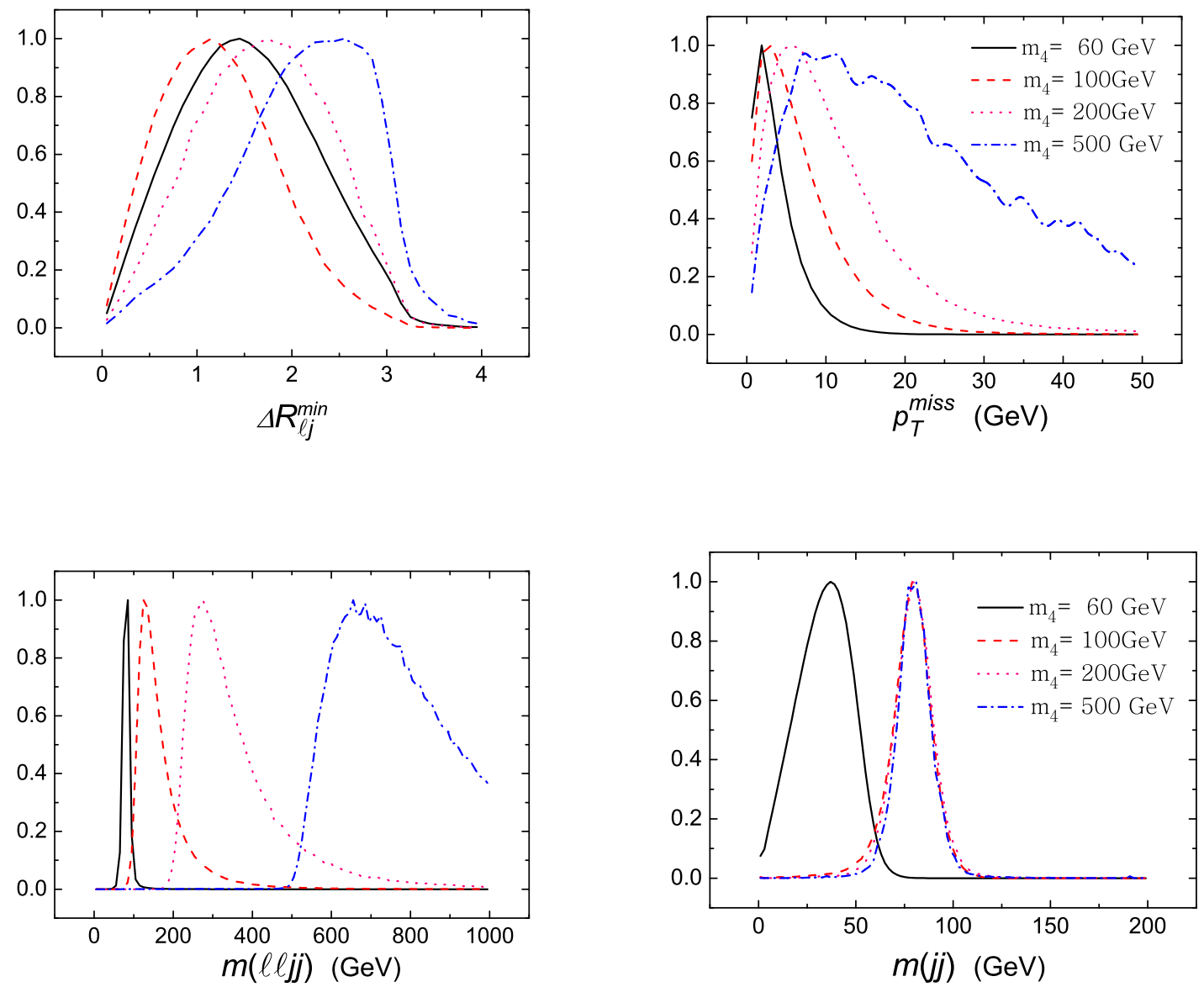

Figure 19: Normalized distributions $\sigma^{-1} d \sigma / d X$ for $m_{4}=60,100,200$ and $500 \mathrm{GeV}$ at the LHC for (a) upper left: the minimal isolation $\Delta R_{\ell j}^{\min }$; (b) upper right: the missing transverse momentum $\not p_{T}$; (c) bottom left: the $2 \ell 2 j$ system invariant mass $m(\ell \ell j j)$; (d) bottom right: the di-jet invariant mass $m(j j)$.

will be scaled down roughly according to this factor for LHC with c.m. energy of $10 \mathrm{TeV}$.

As discussed in the previous section, a large SM background comes mainly from top quark production and decay via the chain decay $t \rightarrow b \rightarrow c \ell^{+} \nu_{\ell}$. Fortunately, after all the selective cuts in Eqs. (4.19) - (4.23), the top-quark decay background is essentially eliminated and has no remaining events for the expected luminosity of $100 \mathrm{fb}^{-1}$ at LHC.

There are several other SM backgrounds coming from like-sign $W$ boson production at the LHC energies. First of all, the triple gauge-boson production process

$$
p p \rightarrow W^{ \pm} W^{ \pm} W^{\mp} \rightarrow \ell^{ \pm} \ell^{ \pm} \nu \nu j j
$$

leads to the irreducible background with two like-sign leptons plus jets. Next, the same final state can be produced via the process

$$
p p \rightarrow W^{ \pm} W^{ \pm} j j \rightarrow \ell^{ \pm} \ell^{ \pm} \nu \nu j j
$$


Table 5: The representative signal and background cross sections at the LHC, for $\mu^{ \pm} \mu^{ \pm} j j$ and the efficiencies with the consecutive cuts. For illustration, we have used $m_{4}=200 \mathrm{GeV},\left|V_{\mu 4}\right|^{2}=$ $\left|V_{\tau 4}\right|^{2}=5 \times 10^{-3} \gg\left|V_{e 4}\right|^{2}$, and $m_{H}=120,300 \mathrm{GeV}$.

\begin{tabular}{|c|c|c|c|c|c|}
\hline & No cut & $\begin{array}{l}\text { Basic cut } \\
(4.19)\end{array}$ & $\begin{array}{c}+\not p_{T} \text { cut } \\
4.23\end{array}$ & $\begin{array}{c}+\Delta R \text { cut } \\
\text { (4.21) }\end{array}$ & $\begin{array}{c}+m(j j), m(\ell \ell j j) \mathrm{cut} \\
(4.22)\end{array}$ \\
\hline $\begin{array}{c}\text { Signal } \\
\sigma(\mathrm{fb}) \\
\text { eff. }\end{array}$ & $\begin{array}{c}0.86 \\
-\end{array}$ & $\begin{array}{l}0.42 \\
48 \%\end{array}$ & $\begin{array}{l}0.37 \\
88 \%\end{array}$ & $\begin{array}{l}0.35 \\
96 \%\end{array}$ & $\begin{array}{l}0.33 \\
94 \%\end{array}$ \\
\hline $\begin{array}{c}t \bar{t} \text { Bkg } \\
\sigma(\mathrm{fb}) \\
\text { eff. }\end{array}$ & $\begin{array}{c}29.6 \\
-\end{array}$ & $\begin{array}{l}16.9 \\
57 \%\end{array}$ & $\begin{array}{c}2.7 \\
16 \%\end{array}$ & $\begin{array}{l}0.075 \\
2.8 \%\end{array}$ & $\begin{array}{l}0.002 \\
2.7 \%\end{array}$ \\
\hline $\begin{array}{c}W^{ \pm} W^{ \pm} W^{\mp} \\
\sigma \\
\text { eff. } \\
\sigma(\mathrm{fb}) \\
\text { eff. }\end{array}$ & $\begin{array}{c}m_{H}=120 \mathrm{GeV} \\
1.01 \\
- \\
m_{H}=300 \mathrm{GeV} \\
1.28 \\
-\end{array}$ & $\begin{array}{l}0.42 \\
42 \% \\
0.58 \\
45 \%\end{array}$ & $\begin{array}{c}0.057 \\
14 \% \\
0.066 \\
11 \%\end{array}$ & $\begin{array}{c}0.052 \\
91 \% \\
0.061 \\
92 \%\end{array}$ & $\begin{array}{c}0.050 \\
96 \% \\
0.058 \\
95 \%\end{array}$ \\
\hline $\begin{array}{c}W^{ \pm} W^{ \pm} j j \\
\sigma(\mathrm{fb}) \\
\text { eff. } \\
\sigma(\mathrm{fb}) \\
\text { eff. }\end{array}$ & $\begin{aligned} m_{H}= & 120 \mathrm{GeV} \\
& 4.2 \\
& - \\
m_{H}= & 300 \mathrm{GeV} \\
& 4.4 \\
& -\end{aligned}$ & $\begin{array}{c}1.3 \\
31 \% \\
1.4 \\
32 \%\end{array}$ & $\begin{array}{l}0.29 \\
22 \% \\
0.34 \\
24 \%\end{array}$ & $\begin{array}{l}0.17 \\
59 \% \\
0.19 \\
56 \%\end{array}$ & $\begin{array}{c}0.019 \\
11 \% \\
0.025 \\
13 \%\end{array}$ \\
\hline
\end{tabular}

where the two jets may come from either QCD scattering or from the gauge-boson fusion process. However these backgrounds have two missing neutrinos and can be suppressed by a combination of cuts on the missing transverse energy and invariant mass. We also analysed the backgrounds coming from $Z$ boson production

$$
p p \rightarrow j j Z Z, \quad p p \rightarrow j j Z W .
$$

in which some charged leptons are missing in the detection so that they lead to like-sign dilepton events. The backgrounds are very small after the cuts.

We list the number of background events and efficiency of cuts in Table 5 for a luminosity of $100 \mathrm{fb}^{-1}$ at LHC. The total background is about $7-8$ events for $100 \mathrm{fb}^{-1}$ at the LHC. The main background is from the $W^{ \pm} W^{ \pm} W^{\mp}$ channel and can be further suppressed if a tighter missing energy cut could be exploited. For instance, the background events may be reduced by half, leaving about $3-4$ events with $p_{T}<15 \mathrm{GeV}$.

The last but not least important feature of the signal is the direct reconstruction of the resonant mass of $N_{4}$ in the final state $\ell^{ \pm} j j$. This is shown in Fig. 21 for the SM background and the signal with $m_{4}=200,400 \mathrm{GeV}$. We see the effective reconstruction of the resonant mass. For a given mass $m_{4}$ in the search, one can further make the event selection on $m(\ell j j)$

$$
0.8 m_{4}<m(\ell j j)<1.2 m_{4},
$$

to estimate the significance of the signal observation. This loose cut has little effect on the signal, but reduces the total background to $0-4$ events for $100 \mathrm{fb}^{-1}$ in the range of 


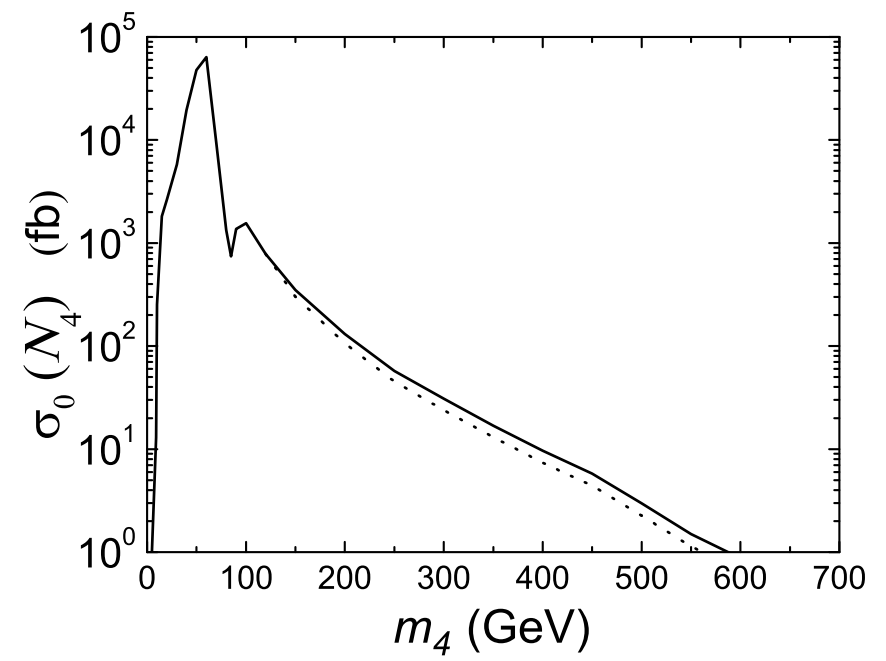

Figure 20: The bare cross section $\sigma_{0}\left(N_{4}\right)$ versus heavy neutrino mass $m_{4}$ after all the cuts at the LHC (14 TeV). The solid (dotted) line correspond to the exclusion (inclusion) of the Higgs decay channel for $m_{H}=120 \mathrm{GeV}$.

$m_{4}$ as shown in Fig. 22. We once again adopt Poisson statistics to determine the search sensitivity. The number of signal events needed for $2 \sigma$ significance would be $3-11$; and $15-44$ for $5 \sigma$ significance. In Fig. 23(a) and Fig. 23(b), we summarize the sensitivity for $S_{\mu \mu}$ and $S_{e \mu}$ versus $m_{4}$, respectively. The solid (dashed) curves correspond to $2 \sigma(5 \sigma)$ limits on $S_{\ell \ell^{\prime}}$ with the exclusion of the Higgs decay channel. The dotted (dash dotted) curves are similar but with the inclusion of the Higgs decay channel for $m_{H}=120 \mathrm{GeV}$. The horizontal dotted line corresponds to constraints on $\left|V_{\mu 4}\right|^{2}<6 \times 10^{-3}$ from precision EW measurements [70]. In Fig. 23(b) the dashed line at the bottom corresponds to the limit from $0 \nu \beta \beta$.

In the optimistic case, we assume that $\left|V_{\tau 4}\right|^{2} \ll\left|V_{\mu 4}\right|^{2}$ and $S_{\mu \mu} \simeq\left|V_{\mu 4}\right|^{2} \leq 6 \times 10^{-3}$. The detection sensitivity on heavy neutrino mass can be

$$
m_{4} \sim \begin{cases}375 \mathrm{GeV} & \text { for } 2 \sigma \\ 250 \mathrm{GeV} & \text { for } 5 \sigma\end{cases}
$$

Or alternatively, the mixing parameter can be probed to

$$
S_{\mu \mu} \sim \begin{cases}7 \times 10^{-7} & \text { for } 2 \sigma \\ 3 \times 10^{-6} & \text { for } 5 \sigma\end{cases}
$$

In particular, even with the very stringent bound on $\left|V_{e 4}\right|^{2}$ from $0 \nu \beta \beta$ as indicated by the dashed curve in Fig. 23(b), one may still have $2 \sigma$ sensitivity if $m_{4} \approx m_{W}$.

Our calculations for hadron colliders have been based on parton-level simulations. A recent study [33] pointed out that there may be other backgrounds to be considered when detector effects are included. One of them is the faked like-sign dileptons from the $b \bar{b}$ cascade decay. The other is due to the QCD multi-jet radiation to degrade the reconstruction of $W \rightarrow j j$. Those backgrounds can not be easily simulated in particular at 


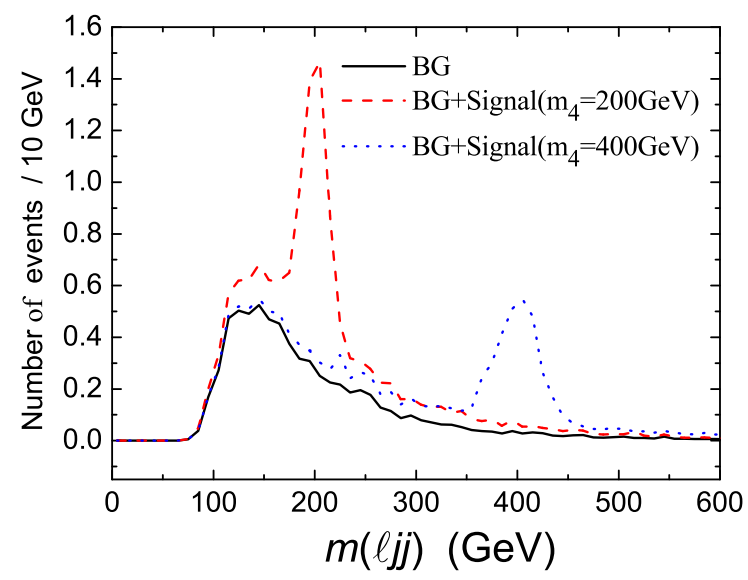

Figure 21: Invariant mass distributions of $m(\ell j j)$ for the signal with $m_{4}=200,400 \mathrm{GeV}$ and background processes.

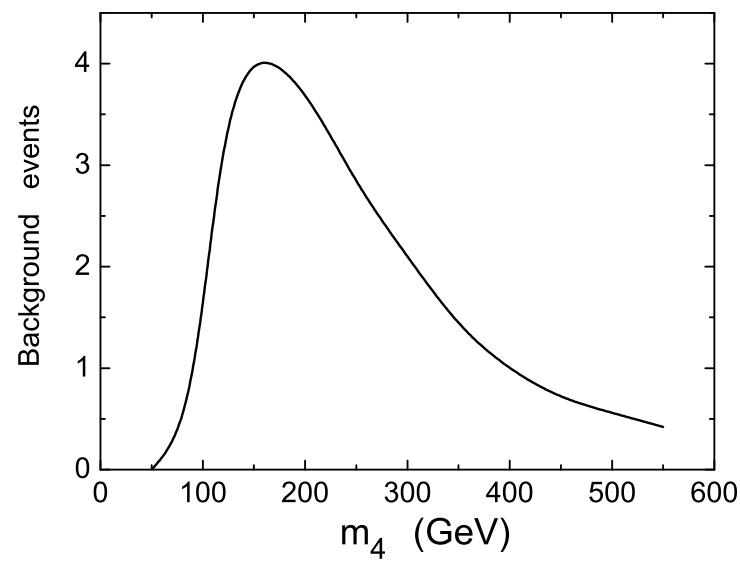

Figure 22: Number of background events vs mass of the heavy neutrino, $m_{4}$.

the parton-level. A preliminary analysis including full CMS detector simulations cannot support their claim [34]. Nevertheless, we may consider to design more stringent acceptance cuts to further discriminate against the backgrounds. First, common wisdom suggests to tighten up the charged lepton isolation requirement

$$
\Delta R_{\ell j}^{\min }>0.8
$$

which would remove the backgrounds from $b, c$ decays substantially, but a full assessment can be made only when real data become available and after the detectors are fully understood. Next, we may increase the jet threshold to suppress the initial state QCD jet radiation to purify the $W \rightarrow j j$ sample. Our estimate based on a PYTHIA simulation shows that the kinematics of a DY-type electroweak process can be largely preserved with 

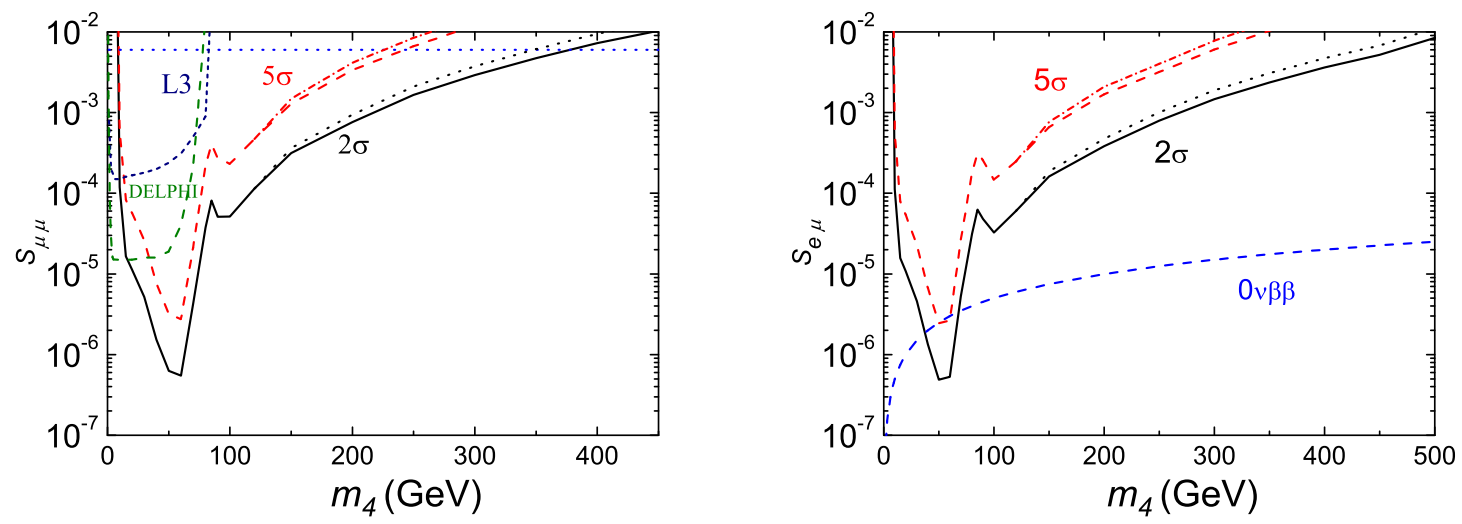

Figure 23: (a) Left: $2 \sigma$ and $5 \sigma$ sensitivity for $S_{\mu \mu}$ versus $m_{4}$ at the LHC with $100 \mathrm{fb}^{-1}$ integrated luminosity; (b) right: same as (a) but for $S_{e \mu}$. The solid and dashed (dotted and dash dotted) curves correspond to limits with the exclusion (inclusion) of the Higgs decay channel for $m_{H}=120$ $\mathrm{GeV}$. The horizontal dotted line corresponds to the constraint on $S_{\mu \mu} \simeq\left|V_{\mu 4}\right|^{2}<6 \times 10^{-3}$ from precision EW measurements [70].
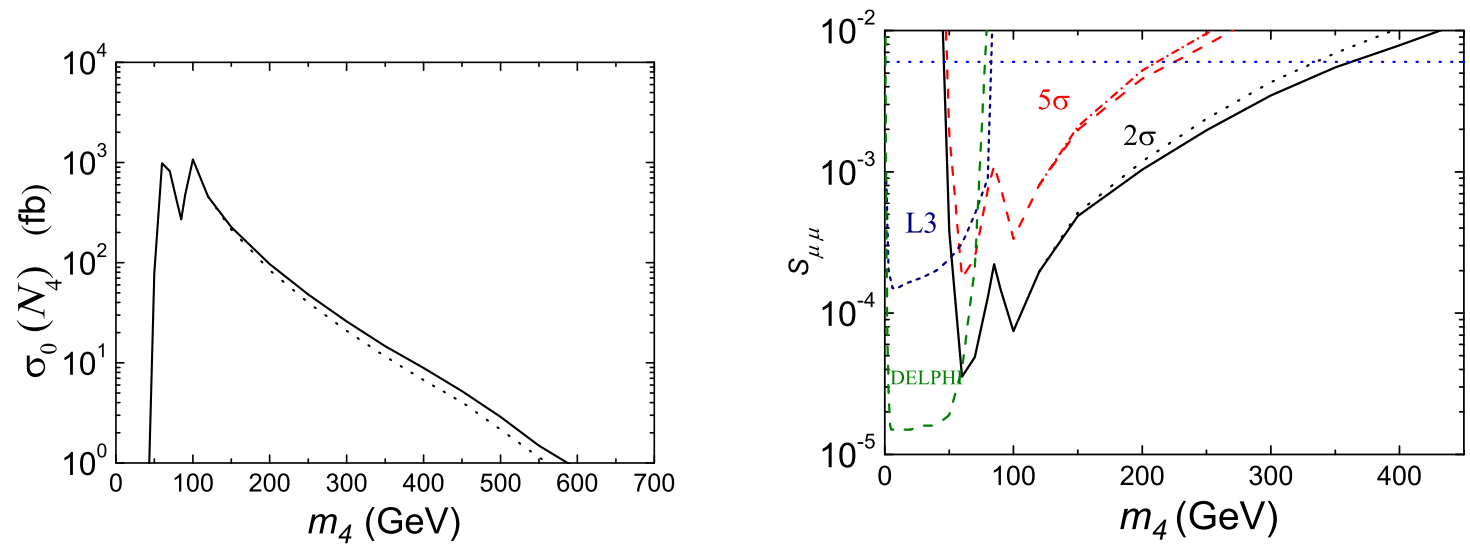

Figure 24: (a) Left: same as Fig. 20 but with the tighter cuts of Eqs. (4.30) and (4.31); (b) right: same as Fig. 23(a) but with the tighter cuts of Eqs. (4.30) and (4.31).

the appropriate jet threshold. We thus examine the cut

$$
p_{T}^{j}>25 \mathrm{GeV},
$$

which results in only about $17 \%$ of the events with potential jet contamination. The results with the tightened cuts are given in Fig. 24. We see that the stringent cuts severely hurt the low mass region, but the effect on the high mass region is modest.

\subsection{Like-sign Dilepton Signals with $\tau$ in the Final States}

So far we have only presented the results with electron and muon final states and ignored the taus. This is due to the experimental challenge of $\tau$ reconstruction. Given the importance 
to cover all the lepton flavors, one must strive to include taus on the search list. Besides the known experimental practice for $\tau$ identification at the Tevatron [108], there are proposals to identify $\tau$ events in connection with the neutrino sector [109. The central issue is to reconstruct the missing momenta from $\tau$ decays. We can generalize our requirement for the charged leptons to the isolated charged tracks presumably from the $\tau$ decays $(e, \mu$, or one-prong and three-prong charged hadrons)

$$
p_{T}(\text { track })>10 \mathrm{GeV}, \quad \mid \eta(\text { track }) \mid<2.5 .
$$

This assures that the parent taus are very energetic. For events with one $\tau$ and no other sources of missing particles, the missing momentum will be along the direction of the charged track. We thus have

$$
\vec{p}(\text { invisible })=\kappa \vec{p}(\text { track })
$$

where the proportionality constant $\kappa$ is determined from the $\mathbb{E}_{T}$ measurement by assigning $E_{T}=\kappa p_{T}$ (track). For events with two taus, we generalize it to

$$
\vec{p}(\text { invisible })=\kappa_{1} \vec{p}\left(\operatorname{track}_{1}\right)+\kappa_{2} \vec{p}\left(\operatorname{track}_{2}\right) .
$$

As long as the two $\tau$ tracks are not linearly dependent, $\kappa_{1}$ and $\kappa_{2}$ can be determined again from the $\not_{T}$ measurement. The missing momenta, as well as the $\tau$ kinematics, are thus fully reconstructed.

Although we believe that the $N_{4}$ signals in the modes of $e^{ \pm} \tau^{ \pm}, \mu^{ \pm} \tau^{ \pm}$and $\tau^{ \pm} \tau^{ \pm}$would be very promising for observation, the background analyses will be considerably more involved due to the complication of $\tau$ reconstruction. Since our simulations are performed at the parton level, we are unable to adequately address the background suppression and to quantify the signal observability. We thus leave this for future studies.

\section{Summary and Conclusions}

The observation of a $L V$ process would show that neutrino is a Majorana particle unambiguously. Apart from light neutrinos, $L V$ processes involving SM particles can receive a contribution from heavy Majorana neutrinos due to mixing. In fact, this contribution can be resonantly enhanced for appropriate masses of the heavy neutrino. In the absence of observation of $L V$ interactions, the rates for these processes can constrain the mixing elements $\left|V_{\ell_{1} 4} V_{\ell_{2} 4}\right|$ as a function of the mass $m_{4}$ of the heavy Majorana neutrino. We considered two classes of $L V$ violating processes: (a) low energy $\Delta L=2$ tau decays and rare meson decays and (b) collider signals for like-sign dilepton production with no missing energy implying the existence of Majorana neutrinos. We emphasize the necessity of involving two charged leptons and no neutrinos in the initial and final states, to be conclusive about lepton-number violation.

For the low energy interactions we evaluated the transition rates and branching fractions as a function of the mass and mixing of the heavy neutrinos. We then translated the current experimental bounds from direct searches into limits on $\left|V_{\ell_{1} 4} V_{\ell_{2} 4}\right|$ as a function of the mass $m_{4}$ of the heavy neutrino. Amongst the rare meson decays, the $K^{+} \rightarrow \ell_{1}^{+} \ell_{2}^{+} \pi^{-}$ decay mode currently gives the most sensitive experimental limits on $\left|V_{\ell_{1} 4} V_{\ell_{2} 4}\right|$. Potentially, these constraints are six orders of magnitude more stringent than the constraints 
from precision electroweak data which limit $\left|V_{\ell 4}\right|^{2}$ to few times $10^{-3}$. As the intermediate heavy sterile neutrino is a real particle which might exit the detector if the decay length is longer than the detector size, for very small mixing angles the bounds get weakened but are still much more stringent than the electroweak precision constraints. This effect should be taken into account and a detailed analysis of past experimental data is required in order to find the precise limits on the mixing angles. Next in sensitivity are the $D$ and $D_{s}$ meson decay modes with constraints of the order of $10^{-3}$. Again, these are competitive with if not better than constraints from EW precision data. The other processes (in other mass ranges) have very weak experimental limits, weaker than EW precision data and essentially do not impose any meaningful bounds on $\left|V_{\ell_{1} 4} V_{\ell_{2} 4}\right|$. This implies that more accurate experimental studies on those rare decays should be strongly encouraged. In particular, many interesting processes of $D, B$ decays have not even been experimentally probed as well as those with a $\tau$ lepton in the final state. Among the $\tau$-decay modes the best limits come from $\tau^{-} \rightarrow \ell^{+} \pi^{-} \pi^{-}$. The other $\tau$-decay modes have sensitivity of order $10^{-3}$ to $10^{-5}$. Again, the constraints from $\tau$ decay modes are competitive with or better than constraints from precision EW data by 2 to 3 orders of magnitude. The experimental bound on $L V$ processes is expected to improve in the future. The future sensitivity of the square of the mixing parameter will increase approximately by an order of magnitude for every order of magnitude improvement in experimental bounds on branching fractions. We have shown that the low energy $\Delta L=2 \tau$ decays and rare meson decays can be very strong probes to discover or constrain the mass and mixing of heavy Majorana neutrinos. Even those decay modes which do not impose strong constraints should not be neglected. It only implies that a large range of the parameter space is available for exploration.

In addition to analyzing the $L V$ tau and meson decay modes and precision EW measurements we also compiled the constraints on the mixing elements $\left(\left|V_{e 4}\right|^{2},\left|V_{\mu 4}\right|^{2}\right.$ and $\left.\left|V_{\tau 4}\right|^{2}\right)$ from peak searches, accelerator experiments, reactor experiments and others - collectively called laboratory constraints. In the absence of detection of $L V$, the laboratory constraints and the ones from $\Delta L=2$ processes can be compared. The constraints on mixing from $L V$ tau decays are always competitive with or better than laboratory constraints in the corresponding mass region while the constraints from $L V$ meson decays are competitive with laboratory constraints only in some mass regions. We note that we explore more combinations of mixing elements and also provide better constraints on mixing in some mass regions. More importantly, a detection in one of the experiments analyzed to obtain laboratory constraints implies the existence of a sterile neutrino while a detection in one of the $\Delta L=2$ tau or meson decay modes studied in our analysis would imply $L V$ and hence the existence of a Majorana neutrino. We pointed out the fact, often ignored in the literature when analyzing low-energy processes, that a heavy neutrino might decay outside the detector if it becomes long-lived for low mass (less than a $\mathrm{GeV}$ ) and/or very small mixing.

For the collider signals of heavy Majorana neutrinos we looked for the definitive leptonnumber violating like-sign dilepton production and no missing energy. Such signals have low backgrounds and have the potential for discovery of heavy Majorana neutrinos. At the Tevatron, with the current and future integrated luminosities, we find that the mass of the heavy Majorana neutrinos can be probed up to

$$
m_{4} \sim\left\{\begin{array}{cc}
10-130 \mathrm{GeV}(10-75 \mathrm{GeV}) & \text { for } 2 \sigma(5 \sigma) \text { with } 2 \mathrm{fb}^{-1} \\
10-180 \mathrm{GeV}(10-120 \mathrm{GeV}) & \text { for } 2 \sigma(5 \sigma) \text { with } 8 \mathrm{fb}^{-1}
\end{array}\right.
$$


Alternatively, the sensitivity for the mixing parameter can be

$$
S_{\mu \mu} \sim\left|V_{\mu 4}\right|^{2} \sim\left\{\begin{array}{cc}
2 \times 10^{-5}\left(10^{-4}\right) & \text { for } 2 \sigma(5 \sigma) \text { with } 2 \mathrm{fb}^{-1} \\
5 \times 10^{-6}\left(2 \times 10^{-5}\right) & \text { for } 2 \sigma(5 \sigma) \text { with } 8 \mathrm{fb}^{-1}
\end{array}\right.
$$

This will surpass the DELPHI [89] and L3 [90, 110] 95\% C.L. bounds.

The sensitivity for heavy Majorana neutrinos can be extended significantly at the LHC. With $100 \mathrm{fb}^{-1}$ of integrated luminosity,

$$
m_{4} \sim 375(250) \mathrm{GeV} \text { for } 2 \sigma(5 \sigma)
$$

or alternatively, the mixing parameter can be probed to

$$
S_{\mu \mu} \sim\left|V_{\mu 4}\right|^{2} \sim 7 \times 10^{-7}\left(3 \times 10^{-6}\right) \text { for } 2 \sigma(5 \sigma) .
$$

The sensitivity at LHC will go well beyond the DELPHI and L3 95\% C.L. bounds in both mass reach and mixing, and beyond the current bound on $\left|V_{e 4}\right|^{2}$ from $0 \nu \beta \beta$. In summary, there is a rich avenue of possibilities for discovering or constraining the elusive Majorana neutrinos.

\section{Acknowledgments}

We would like to thank Pavel Fileviez Perez for comments on the draft. This research was supported in part by the U.S. DOE under Grants No.DE-FG02-95ER40896, W-31-109-Eng38, and in part by the Wisconsin Alumni Research Foundation. Fermilab is operated by Fermi Research Alliance, LLC under Contract No. DE-AC02-07CH11359 with the United States Department of Energy. The work at KITP was supported in part by the National Science Foundation under Grant No. PHY05-51164. The work of B. Z. is supported by the National Science Foundation of China under Grant No. 10705017. SP would like to thank the Theoretical Physics Department at Fermilab and the PH-TH Unit at CERN for hospitality.

\section{A. Lepton mixing formalism}

In this appendix, we illustrate the parameterization for the lepton sector, although we have not followed the relations literally, assuming that new physics beyond this minimal formalism exists. The leptonic content in the theory includes three generations of lefthanded SM $S U(2)_{L}$ doublets and $n$ right-handed SM singlets:

$$
L_{a L}=\left(\begin{array}{c}
\nu_{a} \\
l_{a}
\end{array}\right)_{L}, \quad N_{b R}
$$

where $a=1,2,3$ and $b=1,2,3, \cdots, n$ ( $n \geq 2$ for at least two massive neutrinos).

The leptonically universal gauge interactions involving neutrinos are of the form

$$
-\mathcal{L}=\left(\frac{g}{\sqrt{2}} W_{\mu}^{+} \sum_{a=1}^{3} \overline{\nu_{a L}} \gamma^{\mu} l_{a L}+\text { h.c. }\right)+\frac{g}{2 \cos _{W}} Z_{\mu} \sum_{a=1}^{3} \overline{\nu_{a L}} \gamma^{\mu} \nu_{a L} .
$$


The gauge-invariant Yukawa interactions are

$$
-\mathcal{L}_{Y}=\left(\sum_{a, b=1}^{3} f_{a b}^{l} \overline{L_{a L}} H l_{b R}+\sum_{a=1}^{3} \sum_{b=1}^{n} f_{a b}^{\nu} \overline{L_{a L}} \hat{H} N_{b R}\right)+\text { h.c. }
$$

where $H$ is the SM Higgs doublet and $\hat{H}=i \tau_{2} H^{*}$. After the Higgs field develops a vev $\langle H\rangle \rightarrow v / \sqrt{2}$, the Yukawa interactions lead to Dirac masses for the leptons

$$
-\mathcal{L}_{m}^{D}=\left(\sum_{a, b=1}^{3} \overline{l_{a L}} m_{a b}^{l} l_{b R}+\sum_{a=1}^{3} \sum_{b=1}^{n} \overline{\nu_{a L}} m_{a b}^{\nu} N_{b R}\right)+\text { h.c. }
$$

where the mass matrices are given by the vev times the corresponding Yukawa couplings $m_{a b}^{l, \nu}=f_{a b}^{l, \nu} v / \sqrt{2}$.

The $3 \times 3$ mass matrix $m^{l}$ can be diagonalized by two unitary rotations among the gauge interaction eigenstates $l_{L}, l_{R}$

$$
O_{L}^{\dagger} m^{l} O_{R}=\operatorname{diag}\left(m_{e}, m_{\mu}, m_{\tau}\right), \quad l_{a}=O_{a \ell} \ell,
$$

where $\ell=e, \mu, \tau$ are the mass eigenstates, which define the charged lepton flavors. The Dirac masses as well as interactions with the Higgs boson for the charged leptons now have the standard form

$$
-\mathcal{L}_{Y}^{\ell}=\sum_{\ell=e}^{\tau} m_{\ell}\left(1+\frac{H}{v}\right) \bar{\ell} \ell .
$$

If the Yukawa interactions of Eq. (A.3) are the whole source for neutrino mass, then we would have $\min (n, 3)$ massive Dirac neutrinos.

To complete the neutrino mass sector, there is also a possible heavy Majorana mass term

$$
-\mathcal{L}_{m}^{M}=\frac{1}{2} \sum_{b, b^{\prime}=1}^{n} \overline{N_{b L}^{c}} B_{b b^{\prime}} N_{b^{\prime} R}+\text { h.c. }
$$

where a charge conjugate state is defined as $\psi^{c}=C \bar{\psi}^{T}\left(\overline{\psi^{c}}=\psi^{T} C\right)$, and a chiral state satisfies $\left(\psi^{c}\right)_{\tau}=\left(\psi_{-\tau}\right)^{c}$, with $\tau=-,+$ for $L, R$. The full neutrino mass terms thus read

$$
\begin{aligned}
-\mathcal{L}_{m}^{\nu} & =\frac{1}{2}\left(\sum_{a=1}^{3} \sum_{b=1}^{n}\left(\overline{\nu_{a L}} m_{a b}^{\nu} N_{b R}+\overline{N_{b L}^{c}} m_{b a}^{\nu} \nu_{a R}^{c}\right)+\sum_{b, b^{\prime}=1}^{n} \overline{N_{b L}^{c}} B_{b b^{\prime}} N_{b^{\prime} R}\right)+\text { h.c. } \\
& =\frac{1}{2}\left(\overline{\overline{\nu_{L}}} \overline{N_{L}^{c}}\right)\left(\begin{array}{cc}
0_{3 \times 3} & m_{3 \times n}^{\nu} \\
m_{n \times 3}^{\nu T} & B_{n \times n}
\end{array}\right)\left(\begin{array}{c}
\nu_{R}^{c} \\
N_{R}
\end{array}\right)+\text { h.c. }
\end{aligned}
$$

where we have used the identity $\overline{\nu_{a L}} m_{a b} N_{b R}=\overline{N_{b L}^{c}} m_{b a} \nu_{a R}^{c}$,

The mass matrix can be diagonalized by one unitary transformation

$$
\mathbb{L}^{\dagger}\left(\begin{array}{cc}
0 & m^{\nu} \\
m^{\nu T} & B
\end{array}\right) \mathbb{L}^{*}=\left(\begin{array}{cc}
m_{\text {diag }}^{\nu} & 0 \\
0 & M_{\text {diag }}^{N}
\end{array}\right)
$$

where the mass eigenvalues are of the order

$$
m_{\text {diag }}^{\nu} \approx \frac{m_{\nu}^{2}}{B}, \quad M_{\text {diag }}^{N} \approx B .
$$


$\mathbb{L}$ is a $(3+n) \times(3+n)$ unitary matrix and can be parameterized as

$$
\mathbb{L}=\left(\begin{array}{cc}
U_{3 \times 3} & V_{3 \times n} \\
X_{n \times 3} & Y_{n \times n}
\end{array}\right)
$$

The relation between the gauge interaction eigenstates and the mass eigenstates are given by

$$
\left(\begin{array}{c}
\nu_{L} \\
N_{L}^{c}
\end{array}\right)=\mathbb{L}\left(\begin{array}{c}
\nu_{L} \\
N_{L}^{c}
\end{array}\right)_{m}
$$

with the mass eigenstates $\nu_{m}(m=1,2,3), N_{m^{\prime}}\left(m^{\prime}=4, \cdots, 3+n\right)$. The diagonalized (Majorana) mass terms of Eq. (A.8) thus read

$$
-\mathcal{L}_{m}^{\nu}=\frac{1}{2}\left(\sum_{m=1}^{3} m_{m}^{\nu} \overline{\nu_{m L}} \nu_{m R}^{c}+\sum_{m^{\prime}=4}^{3+n} M_{m^{\prime}}^{N} \overline{N_{m^{\prime} L}^{c}} N_{m^{\prime} R}\right)+\text { h.c. },
$$

with the mixing relations between the gauge and mass eigenstates

$$
\begin{aligned}
& \nu_{a L}=\sum_{m=1}^{3} U_{a m} \nu_{m L}+\sum_{m^{\prime}=4}^{3+n} V_{a m^{\prime}} N_{m^{\prime} L}^{c}, \quad N_{b L}^{c}=\sum_{m=1}^{3} X_{b m} \nu_{m L}+\sum_{m^{\prime}=4}^{3+n} Y_{b m^{\prime}} N_{m^{\prime} L}^{c},(A) \\
& \nu_{a R}^{c}=\sum_{m=1}^{3} U_{a m}^{*} \nu_{m R}^{c}+\sum_{m^{\prime}=4}^{3+n} V_{a m^{\prime}}^{*} N_{m^{\prime} R}, \quad N_{b R}=\sum_{m=1}^{3} X_{b m}^{*} \nu_{m R}^{c}+\sum_{m^{\prime}=4}^{3+n} Y_{b m^{\prime}}^{*} N_{m^{\prime} R} .(A)
\end{aligned}
$$

Note that the unitarity condition for $\mathbb{L}$ leads to the relations

$$
\begin{aligned}
& U U^{\dagger}+V V^{\dagger}=U^{\dagger} U+X^{\dagger} X=I_{3 \times 3}, \\
& X X^{\dagger}+Y Y^{\dagger}=V^{\dagger} V+Y^{\dagger} Y=I_{n \times n} .
\end{aligned}
$$

Parametrically, $U U^{\dagger}$ and $Y^{\dagger} Y \sim \mathcal{O}(1), V V^{\dagger}$ and $X^{\dagger} X \sim \mathcal{O}\left(m_{\nu} / M_{N}\right)$.

In terms of the mass eigenstates, the gauge interaction lagrangian Eq. (A.2) can be written as

$$
\begin{aligned}
-\mathcal{L} & =\frac{g}{\sqrt{2}} W_{\mu}^{+}\left(\sum_{\ell=e}^{\tau} \sum_{m=1}^{3}\left(U^{\dagger} O_{L}\right)_{m \ell} \overline{\nu_{m}} \gamma^{\mu} P_{L} \ell+\sum_{\ell=e}^{\tau} \sum_{m^{\prime}=4}^{3+n}\left(V^{\dagger} O_{L}\right)_{m^{\prime} \ell} \overline{N_{m^{\prime}}^{c}} \gamma^{\mu} P_{L} \ell\right)+\text { h.c. } \\
& +\frac{g}{2 \cos _{W}} Z_{\mu}\left(\sum_{m_{1}, m_{2}=1}^{3}\left(U^{\dagger} U\right)_{m_{1} m_{2}} \overline{\nu_{m_{1}}} \gamma^{\mu} P_{L} \nu_{m_{2}}+\sum_{m_{1}^{\prime}, m_{2}^{\prime}=4}^{3+n}\left(V^{\dagger} V\right)_{m_{1}^{\prime} m_{2}^{\prime}} \overline{N_{m_{1}^{\prime}}^{c}} \gamma^{\mu} P_{L} N_{m_{2}^{\prime}}^{c}\right) \\
& +\frac{g}{2 \cos _{W}} Z_{\mu}\left(\sum_{m_{1}=1}^{3} \sum_{m_{2}^{\prime}=4}^{3+n}\left(U^{\dagger} V\right)_{m_{1}, m_{2}^{\prime}} \overline{\nu_{m_{1}}} \gamma^{\mu} P_{L} N_{m_{2}^{\prime}}^{c}+\text { h.c. }\right) .
\end{aligned}
$$

To make the couplings more intuitive, we define the combination matrices by

$$
U^{l \nu}=O_{L}^{\dagger} U, \quad V^{l N}=O_{L}^{\dagger} V, \quad U^{\nu N}=U^{\dagger} V, \quad U^{\nu \nu}=U^{\dagger} U, \quad V^{N N}=V^{\dagger} V .
$$

We thus rewrite the gauge interaction lagrangian by one mixing matrix for each term

$$
-\mathcal{L}=\frac{g}{\sqrt{2}} W_{\mu}^{+}\left(\sum_{\ell=e}^{\tau} \sum_{m=1}^{3} U_{\ell m}^{l \nu *} \overline{\nu_{m}} \gamma^{\mu} P_{L} \ell+\sum_{\ell=e}^{\tau} \sum_{m^{\prime}=4}^{3+n} V_{\ell m^{\prime}}^{l N *} \overline{N_{m^{\prime}}^{c}} \gamma^{\mu} P_{L} \ell\right)+\text { h.c. }
$$



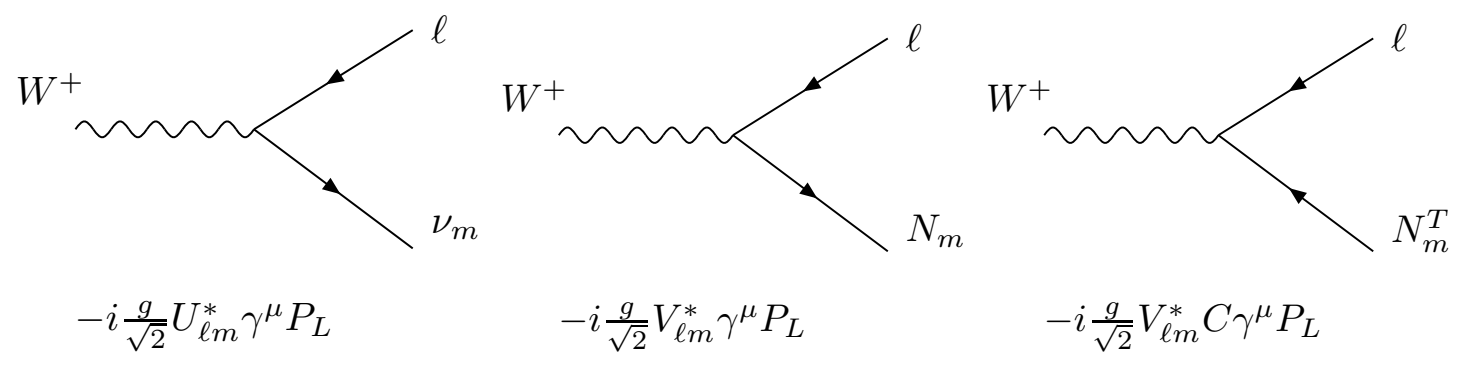

Figure 25: Feynman rules for the charged current vertices in terms of the neutrino mass eigenstates, as given in Eq. (A.22).

$$
\begin{aligned}
& +\frac{g}{2 \cos _{W}} Z_{\mu}\left(\sum_{m_{1}, m_{2}=1}^{3} U_{m_{1} m_{2}}^{\nu \nu} \overline{\nu_{m_{1}}} \gamma^{\mu} P_{L} \nu_{m_{2}}+\sum_{m_{1}^{\prime}, m_{2}^{\prime}=4}^{3+n} V_{m_{1}^{\prime} m_{2}^{\prime}}^{N N} \overline{N_{m_{1}^{\prime}}} \gamma^{\mu} P_{L} N_{m_{2}^{\prime}}\right) \\
& +\frac{g}{2 \cos _{W}} Z_{\mu}\left(\sum_{m_{1}=1}^{3} \sum_{m_{2}^{\prime}=4}^{3+n} U_{m_{1} m_{2}^{\prime}}^{\nu N} \overline{\nu_{m_{1}}} \gamma^{\mu} P_{L} N_{m_{2}^{\prime}}^{c}+\text { h.c. }\right) .
\end{aligned}
$$

These couplings along with the mixing matrices Eq. (A.19) give the most general leptonic interactions of the charged and neutral currents in terms of the mass eigenstates. Alternatively, the neutral current interactions can be aligned along with that of the charged currents when rotating left-handed neutrinos in the same way as the charged leptons,

$$
\nu_{a L}=\left(O_{L}\right)_{a \ell} \nu_{\ell L}, \text { or } \nu_{\ell L}=\sum_{m=1}^{3}\left(O_{L}^{\dagger} U\right)_{\ell m} \nu_{m L}+\sum_{m^{\prime}=4}^{3+n}\left(O_{L}^{\dagger} V\right)_{\ell m^{\prime}} N_{m^{\prime} L}^{c} .
$$

It may be convenient in certain practical calculations to rewrite the neutral current interactions in terms of their flavor eigenstates

$$
\begin{aligned}
-\mathcal{L} & =\frac{g}{\sqrt{2}} W_{\mu}^{+}\left(\sum_{\ell=e}^{\tau} \sum_{m=1}^{3} U_{\ell m}^{*} \overline{\nu_{m}} \gamma^{\mu} P_{L} \ell+\sum_{\ell=e}^{\tau} \sum_{m^{\prime}=4}^{3+n} V_{\ell m^{\prime}}^{*} \overline{N_{m^{\prime}}^{c}} \gamma^{\mu} P_{L} \ell\right)+\text { h.c. } \\
& +\frac{g}{2 \cos \theta_{W}} Z_{\mu}\left(\sum_{\ell=e}^{\tau} \sum_{m=1}^{3} U_{\ell m}^{*} \overline{\nu_{m}} \gamma^{\mu} P_{L} \nu_{\ell}+\sum_{\ell=e}^{\tau} \sum_{m^{\prime}=4}^{3+n} V_{\ell m^{\prime}}^{*} \overline{N_{m^{\prime}}^{c}} \gamma^{\mu} P_{L} \nu_{\ell}\right)+\text { h.c. }+.(\mathrm{A} .
\end{aligned}
$$

where we have dropped the superscripts for $U, V$ defined in Eq. (A.19), for simplicity as adopted throughout the text.

For the reader's convenience, we give most of the corresponding Feynman rules for the interaction vertices, listed in Fig. 25 for the charged currents, and in Fig. 26 for the neutral currents. The Feynman rules for the other diagrams can be easily deduced from the ones that are explicitly written down in Fig. 25 and Fig. 26.

Finally, the heavy neutrino interactions with the Higgs boson read

$$
-\mathcal{L}_{\mathcal{H}}=\frac{H}{v} \sum_{\ell=e}^{\tau} \sum_{m^{\prime}=4}^{3+n} V_{\ell m^{\prime}}^{*} M_{m^{\prime}}^{N} \overline{N_{m^{\prime}}^{c}} P_{L} \nu_{\ell}+\text { h.c. }+\ldots
$$

The corresponding Feynman rule for the interaction vertex is given in Fig. 27. 

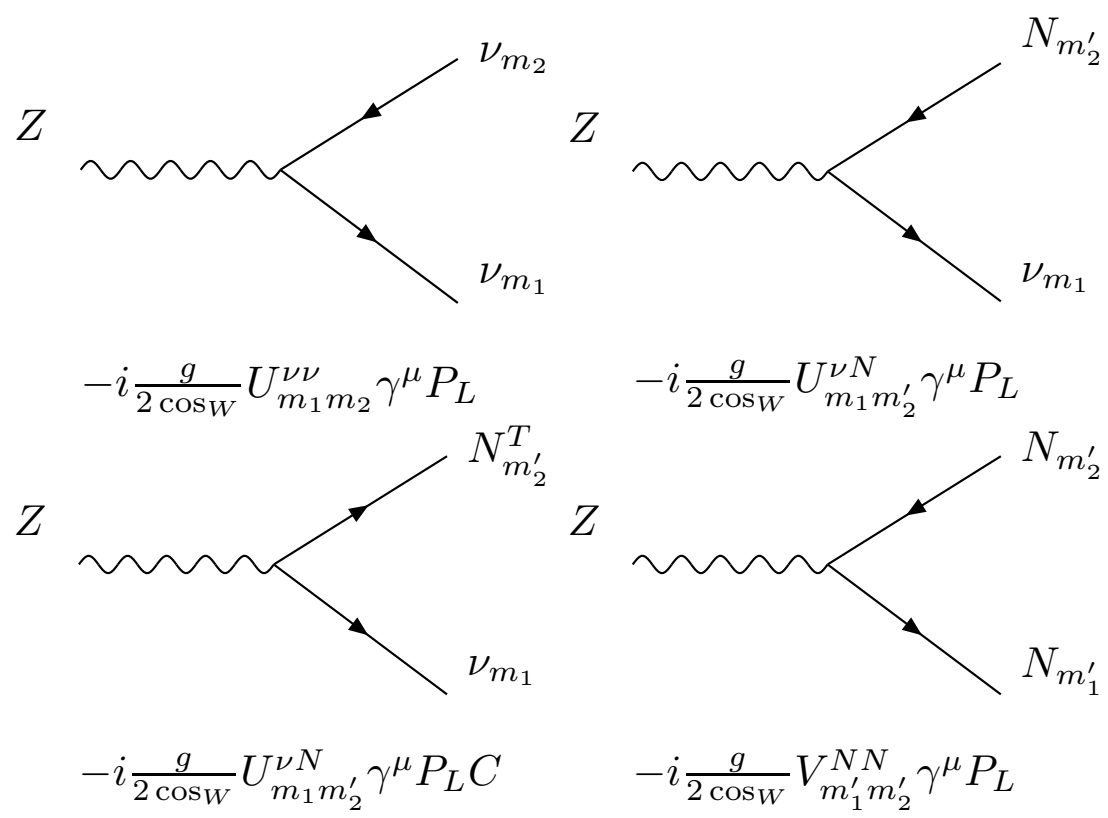

Figure 26: Feynman rules for the neutral current vertices in terms of the neutrino mass eigenstates, as given in Eqs. (A.20).
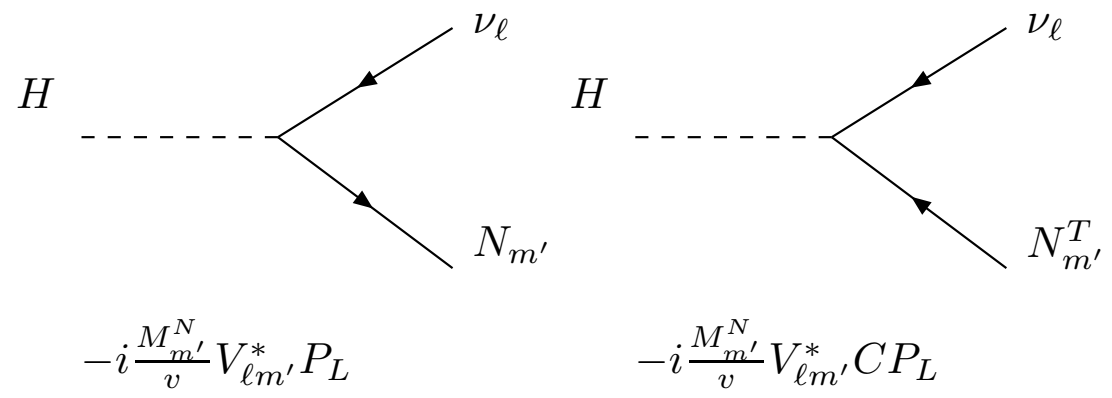

Figure 27: Feynman rule for the Higgs vertex in terms of the heavy neutrino mass eigenstates, as given in Eq. (A.23).

\section{B. General amplitude of $\Delta L=2$ processes}

The charged current interaction lagrangian in terms of neutrino mass eigenstates is

$$
\mathcal{L}_{c c}=-\frac{g}{\sqrt{2}} W_{\mu}^{+}\left(\sum_{\ell=e}^{\tau} \sum_{m=1}^{3} U_{\ell m}^{l \nu *} \overline{\nu_{m}} \gamma^{\mu} P_{L} \ell+\sum_{\ell=e}^{\tau} \sum_{m^{\prime}=4}^{3+n} V_{\ell m^{\prime}}^{l N *} \overline{N_{m^{\prime}}^{c}} \gamma^{\mu} P_{L} \ell\right)+\text { h.c. }
$$

where $P_{L}=\frac{1}{2}\left(1-\gamma_{5}\right)$. The leptonic $\Delta L=2$ subprocess $W^{-} W^{-} \rightarrow \ell_{1}^{-} \ell_{2}^{-}$is induced by the product of two charged currents

$\mathcal{M}_{l e p}^{\mu \nu} \propto \sum_{m=1}^{3} U_{\ell_{1} m}^{l \nu} U_{\ell_{2} m}^{l \nu}\left(\overline{\ell_{1}} \gamma^{\mu} P_{L} \nu_{m}\right)\left(\overline{\ell_{2}} \gamma^{\nu} P_{L} \nu_{m}\right)+\sum_{m^{\prime}=4}^{3+n} V_{\ell_{1} m^{\prime}}^{l N} V_{\ell_{2} m^{\prime}}^{l N}\left(\overline{\ell_{1}} \gamma^{\mu} P_{L} N_{m^{\prime}}\right)\left(\overline{\ell_{2}} \gamma^{\nu} P_{L} N_{m^{\prime}}\right)$, 
which can be rewritten using charge conjugation as

$\mathcal{M}_{l e p}^{\mu \nu} \propto \sum_{m=1}^{3} U_{\ell_{1} m}^{l \nu} U_{\ell_{2} m}^{l \nu}\left(\overline{\ell_{1}} \gamma^{\mu} P_{L} \nu_{m}\right)\left(\overline{\nu_{m}} \gamma^{\nu} P_{R} \ell_{2}^{c}\right)+\sum_{m^{\prime}=4}^{3+n} V_{\ell_{1} m^{\prime}}^{l N} V_{\ell_{2} m^{\prime}}^{l N}\left(\overline{\ell_{1}} \gamma^{\mu} P_{L} N_{m^{\prime}}\right)\left(\overline{N_{m^{\prime}}} \gamma^{\nu} P_{R} \ell_{2}^{c}\right)$.

The Majorana neutrino fields can be contracted to form a neutrino propagator, and the transition matrix element is thus given by

$$
\begin{aligned}
\mathcal{M}_{l e p}^{\mu \nu} & =\frac{g^{2}}{2} \sum_{m=1}^{3} U_{\ell_{1} m}^{l \nu} U_{\ell_{2} m}^{l \nu}\left(\overline{\ell_{1}} \gamma^{\mu} P_{L}\right) \frac{q+m_{\nu_{m}}}{q^{2}-m_{\nu_{m}}^{2}+i \Gamma_{\nu_{m}} m_{\nu_{m}}}\left(\gamma^{\nu} P_{R} \ell_{2}^{c}\right) \\
& +\frac{g^{2}}{2} \sum_{m^{\prime}=4}^{3+n} V_{\ell_{1} m^{\prime}}^{l N} V_{\ell_{2} m^{\prime}}^{l N}\left(\overline{\ell_{1}} \gamma^{\mu} P_{L}\right) \frac{q+m_{N_{m^{\prime}}}}{q^{2}-m_{N_{m^{\prime}}}^{2}+i \Gamma_{N_{m^{\prime}}} m_{N_{m^{\prime}}}}\left(\gamma^{\nu} P_{R} \ell_{2}^{c}\right),
\end{aligned}
$$

where $q$ is the momentum exchange carried by the neutrino. The $q$ term vanishes due to the chirality flip. Including the crossed diagram $\left(\ell_{1} \leftrightarrow \ell_{2}\right)$ the leptonic amplitude then becomes

$$
\begin{aligned}
\mathcal{M}_{l e p}^{\mu \nu}= & \frac{g^{2}}{2} \sum_{m=1}^{3} U_{\ell_{1} m}^{l \nu} U_{\ell_{2} m}^{l \nu} m_{\nu_{m}} \overline{u_{1}}\left(\frac{\gamma^{\mu} \gamma^{\nu}}{q^{2}-m_{\nu_{m}}^{2}+i \Gamma_{\nu_{m}} m_{\nu_{m}}}+\frac{\gamma^{\nu} \gamma^{\mu}}{q^{\prime 2}-m_{\nu_{m}}^{2}+i \Gamma_{\nu_{m}} m_{\nu_{m}}}\right) P_{R} v_{2} \\
+ & \frac{g^{2}}{2} \sum_{m^{\prime}=4}^{3+n} V_{\ell_{1} m^{\prime}}^{l N} V_{\ell_{2} m^{\prime}}^{l N} m_{N_{m^{\prime}}} \times \\
& \overline{u_{1}}\left(\frac{\gamma^{\mu} \gamma^{\nu}}{q^{2}-m_{N_{m^{\prime}}}^{2}+i \Gamma_{N_{m^{\prime}}} m_{N_{m^{\prime}}}}+\frac{\gamma^{\nu} \gamma^{\mu}}{q^{\prime 2}-m_{N_{m^{\prime}}}^{2}+i \Gamma_{N_{m^{\prime}}} m_{N_{m^{\prime}}}}\right) P_{R} v_{2} .
\end{aligned}
$$

For the light Majorana neutrinos, namely, $m=1,2,3$ the masses $m_{\nu_{m}} \sim \mathcal{O}(\mathrm{eV})$ 111 and for the heavy Majorana neutrinos, the masses $m_{N_{m^{\prime}}} \sim \mathcal{O}(\mathrm{MeV}-\mathrm{GeV})$ for the low energy processes we consider. The heavy Majorana neutrino contribution has a resonant enhancement when $q^{2}, q^{2} \approx m_{N_{m^{\prime}}}^{2}$ and is the dominant one. The light Majorana neutrino contribution however encounters a severe suppression due to the small neutrino mass like $m_{\nu_{m}}^{2} / M_{W}^{2}$. Hence we can neglect the contributions of the light Majorana neutrinos and the $\sum_{m=1}^{3}$ part of the amplitude drops out.

In principle all the heavy Majorana neutrinos will contribute to the amplitude but in our analysis we only consider the contribution of one of the heavy neutrinos, in particular the lightest one for simplicity. So the amplitude can now be written as

$$
\mathcal{M}_{\text {lep }}^{\mu \nu}=\frac{g^{2}}{2} V_{\ell_{1} 4} V_{\ell_{2} 4} m_{4} \overline{u_{1}}\left(\frac{\gamma^{\mu} \gamma^{\nu}}{q^{2}-m_{4}^{2}+i \Gamma_{N_{4}} m_{4}}+\frac{\gamma^{\nu} \gamma^{\mu}}{q^{\prime 2}-m_{4}^{2}+i \Gamma_{N_{4}} m_{4}}\right) P_{R} v_{2} .
$$

We can rewrite the above amplitude as

$$
\begin{aligned}
\mathcal{M}_{\text {lep }}^{\mu \nu} & =\frac{g^{2}}{2} V_{\ell_{1} 4} V_{\ell_{2} 4} m_{4} \frac{\overline{u_{1}} \gamma^{\mu} \gamma^{\nu} P_{R} v_{2}}{q^{2}-m_{4}^{2}+i \Gamma_{N_{4}} m_{4}}+\frac{g^{2}}{2} V_{\ell_{1} 4} V_{\ell_{2} 4} m_{4} \frac{\overline{u_{1}} \gamma^{\nu} \gamma^{\mu} P_{R} v_{2}}{q^{2}-m_{4}^{2}+i \Gamma_{N_{4}} m_{4}} \\
& =\mathcal{M}_{1}+\mathcal{M}_{2} .
\end{aligned}
$$

When $q^{2} \approx m_{4}^{2}, \mathcal{M}_{1}$ has a resonant contribution and when $q^{\prime 2} \approx m_{4}^{2}, \mathcal{M}_{2}$ has a resonant contribution. In general, $q \neq q^{\prime}$, and it is convenient to split up the individual resonant 
contributions by the Single-Diagram-Enhanced multi-channel integration method [112]. To do this, define the functions

$$
f_{i}=\frac{\left|\mathcal{M}_{i}\right|^{2}}{\sum_{i}\left|\mathcal{M}_{i}\right|^{2}}\left|\sum_{i} \mathcal{M}_{i}\right|^{2}
$$

Then the amplitude squared is given by

$$
\left|\sum_{i} \mathcal{M}_{i}\right|^{2}=\sum_{i} f_{i}
$$

The amplitude squared splits up into the functions $f_{i}$ defined above and the phase space integration can be done for each $f_{i}$ separately. This helps to make convenient simplifications for the phase space integration and the computation can be carried out in parallel. The contributions from each $f_{i}$ can be added up after phase space integration.

\section{Decay modes of heavy Majorana neutrino}

In this section we will discuss in detail the decay modes of the heavy Majorana neutrino $N_{4}$, with mass $m_{4}$ much smaller than the mass of the $\mathrm{W}$ boson, $m_{W}$. From EW precision measurements the mixing elements $\left|V_{\ell 4}\right|^{2} \lesssim \mathcal{O}\left(10^{-3}\right)$ and the higher order terms in mixing would be very small and can be ignored. Hence the widths are presented only up to leading terms in mixing. The charged current and neutral current vertices of $N_{4}$ with the mixing elements are given in Fig. 25 and Fig. 26. With increasing mass of the heavy neutrino new decay channels open up and can be classified into two body and three body decays. The decay width scales as the third and the fifth power of the mass $\left(m_{4}\right)$ for two and three body decays respectively.

1) $N_{4} \rightarrow \ell^{-} P^{+}$where $\ell=e, \mu, \tau$ and $P^{+}$is a charged pseudoscalar meson. This decay mode has charged current interactions only as shown in Fig. 25 and the decay width is given by

$$
\begin{aligned}
\Gamma^{\ell P} & \equiv \Gamma\left(N_{4} \rightarrow \ell^{-} P^{+}\right)=\frac{G_{F}^{2}}{16 \pi} f_{P}^{2}\left|V_{q \bar{q}^{\prime}}\right|^{2}\left|V_{\ell 4}\right|^{2} m_{4}^{3} I_{1}\left(\mu_{\ell}, \mu_{P}\right), \\
I_{1}(x, y) & =[(1+x-y)(1+x)-4 x] \lambda^{\frac{1}{2}}(1, x, y), \\
\lambda(a, b, c) & =a^{2}+b^{2}+c^{2}-2 a b-2 b c-2 c a,
\end{aligned}
$$

where $f_{P}$ is the meson decay constant and $V_{q \bar{q}^{\prime}}$ are the CKM matrix elements. $\mu_{\ell}$ and $\mu_{P}$ are the masses scaled by the mass of the heavy neutrino and are given by $\mu_{i}=m_{i}^{2} / m_{4}^{2}$.

2) $N_{4} \rightarrow \nu_{\ell} P^{0}$ where $\nu_{\ell}=\nu_{e}, \nu_{\mu}, \nu_{\tau}$ and $P^{0}$ is a neutral pseudoscalar meson. This decay mode has neutral current interactions only as shown in Fig. 26 and the decay width is given by

$$
\Gamma^{\nu_{\ell} P} \equiv \Gamma\left(N_{4} \rightarrow \nu_{\ell} P^{0}\right)=\frac{G_{F}^{2}}{64 \pi} f_{P}^{2}\left|V_{\ell 4}\right|^{2} m_{4}^{3}\left(1-\mu_{P}\right)^{2},
$$

where $f_{P}$ is the meson decay constant, $\mu_{P}$ is the mass of the neutral meson scaled by the mass of the heavy neutrino and is given by $\mu_{P}=m_{P}^{2} / m_{4}^{2}$. The mass of the light neutrino $\sim \mathcal{O}(\mathrm{eV})[18]$ is much smaller than the mass of $N_{4} \sim \mathcal{O}(\mathrm{MeV}-\mathrm{GeV})$ and can be neglected to a very good approximation. We have set the mass of the light neutrino to zero in the expression for the width above and henceforth. 
3) $N_{4} \rightarrow \ell^{-} V^{+}$where $\ell=e, \mu, \tau$ and $V^{+}$is a charged vector meson. This decay mode has charged current interactions only as shown in Fig. 25 and the decay width is given by

$$
\begin{aligned}
\Gamma^{\ell V} & \equiv \Gamma\left(N_{4} \rightarrow \ell^{-} V^{+}\right)=\frac{G_{F}^{2}}{16 \pi} f_{V}^{2}\left|V_{q \bar{q}^{\prime}}\right|^{2}\left|V_{\ell 4}\right|^{2} m_{4}^{3} I_{2}\left(\mu_{\ell}, \mu_{V}\right), \\
I_{2}(x, y) & =[(1+x-y)(1+x+2 y)-4 x] \lambda^{\frac{1}{2}}(1, x, y),
\end{aligned}
$$

where $f_{V}$ is the vector meson decay constant and $V_{q \bar{q}^{\prime}}$ are the CKM matrix elements. $\mu_{\ell}$ and $\mu_{V}$ are the masses of the lepton and the vector meson scaled by the mass of the heavy neutrino and are given by $\mu_{i}=m_{i}^{2} / m_{4}^{2}$.

4) $N_{4} \rightarrow \nu_{\ell} V^{0}$ where $\nu_{\ell}=\nu_{e}, \nu_{\mu}, \nu_{\tau}$ and $V^{0}$ is a neutral vector meson. This decay mode has neutral current interactions only as shown in Fig. 26 and the decay width is given by

$$
\begin{aligned}
\Gamma^{\nu_{\ell} V} & \equiv \Gamma\left(N_{4} \rightarrow \nu_{\ell} V^{0}\right)=\frac{G_{F}^{2}}{2 \pi} \kappa_{V}^{2} f_{V}^{2}\left|V_{\ell 4}\right|^{2} m_{4}^{3} I_{3}\left(\mu_{\nu_{\ell}}, \mu_{V}\right), \\
I_{3}(x, y) & =(1+2 y)(1-y) \lambda^{\frac{1}{2}}(1, x, y),
\end{aligned}
$$

where $f_{V}$ is the meson decay constant, $\mu_{V}$ is the mass of the neutral meson scaled by the mass of the heavy neutrino and is given by $\mu_{V}=m_{V}^{2} / m_{4}^{2} . \kappa_{V}$ is the vector coupling associated with the meson and is expressed in terms of $x_{w}=\sin ^{2} \theta_{w}$, where $\theta_{w}$ is the Weinberg angle. The values of $\kappa$ for the various vector mesons are: $\kappa=\frac{1}{3} x_{w}$ for $\rho^{0}$ and $\omega$; $\kappa=\left(-\frac{1}{4}+\frac{1}{3} x_{w}\right)$ for $K^{* 0}, \bar{K}^{* 0}$ and $\phi$; and $\kappa=\left(\frac{1}{4}-\frac{2}{3} x_{w}\right)$ for $D^{* 0}, \bar{D}^{* 0}$ and $J / \psi$.

5) $N_{4} \rightarrow \ell_{1}^{-} \ell_{2}^{+} \nu_{\ell_{2}}$ where $\ell_{1}, \ell_{2}=e, \mu, \tau$ with $\ell_{1} \neq \ell_{2}$. This decay mode has charged current interactions only as shown in Fig. 25 and the decay width is given by

$$
\begin{aligned}
\Gamma^{\ell_{1} \ell_{2} \nu_{\ell_{2}}} & \equiv \Gamma\left(N_{4} \rightarrow \ell_{1}^{-} \ell_{2}^{+} \nu_{\ell_{2}}\right)=\frac{G_{F}^{2}}{192 \pi^{3}} m_{4}^{5}\left|V_{\ell_{1} 4}\right|^{2} I_{1}\left(x_{\ell_{1}}, x_{\nu_{\ell_{2}}}, x_{\ell_{2}}\right), \\
I_{1}(x, y, z) & =12 \int_{(x+y)^{2}}^{(1-z)^{2}} \frac{d s}{s}\left(s-x^{2}-y^{2}\right)\left(1+z^{2}-s\right) \lambda^{\frac{1}{2}}\left(s, x^{2}, y^{2}\right) \lambda^{\frac{1}{2}}\left(1, s, z^{2}\right),
\end{aligned}
$$

where $I_{1}(0,0,0)=1, x_{i}$ are the masses scaled by the mass of the heavy neutrino and are given by $x_{i}=m_{i} / m_{4}$. The mass of the light neutrino $\sim \mathcal{O}(\mathrm{eV})$ is much smaller than the mass of $N_{4} \sim \mathcal{O}(\mathrm{MeV}-\mathrm{GeV})$ and hence can be neglected compared to the mass of $N_{4}$. We have set the mass of the light neutrino to zero with very good approximation in the expression for the width above and henceforth.

6) $N_{4} \rightarrow \nu_{\ell_{1}} \ell_{2}^{-} \ell_{2}^{+}$where $\ell_{1}, \ell_{2}=e, \mu, \tau$. Both charged current and neutral current interactions as shown in Fig. 25 and Fig. 26 are relevant for this mode and the decay width is given by

$$
\begin{aligned}
\Gamma^{\nu_{1} \ell_{2} \ell_{2}} & \equiv \Gamma\left(N_{4} \rightarrow \nu_{\ell_{1}} \ell_{2}^{-} \ell_{2}^{+}\right)=\frac{G_{F}^{2}}{96 \pi^{3}}\left|V_{\ell_{1} 4}\right|^{2} m_{4}^{5} \times\left[\left(g_{L}^{\ell} g_{R}^{\ell}+\delta_{\ell_{1} \ell_{2}} g_{R}^{\ell}\right) I_{2}\left(x_{\nu_{\ell_{1}}}, x_{\ell_{2}}, x_{\ell_{2}}\right)\right. \\
& \left.+\left(\left(g_{L}^{\ell}\right)^{2}+\left(g_{R}^{\ell}\right)^{2}+\delta_{\ell_{1} \ell_{2}}\left(1+2 g_{L}^{\ell}\right)\right) I_{1}\left(x_{\nu_{\ell_{1}}}, x_{\ell_{2}}, x_{\ell_{2}}\right)\right] \\
I_{2}(x, y, z) & =24 y z \int_{(y+z)^{2}}^{(1-x)^{2}} \frac{d s}{s}\left(1+x^{2}-s\right) \lambda^{\frac{1}{2}}\left(s, y^{2}, z^{2}\right) \lambda^{\frac{1}{2}}\left(1, s, x^{2}\right),
\end{aligned}
$$


where $I_{2}(0,0,0)=1, I_{1}(x, y, z)$ has been defined in Eq. C.5), $x_{i}$ are the masses scaled by the mass of the heavy neutrino and are given by $x_{i}=m_{i} / m_{4}, g_{L}^{\ell}=-\frac{1}{2}+x_{w}, g_{R}^{\ell}=x_{w}$ and $x_{w}=\sin ^{2} \theta_{w}=0.231$, where $\theta_{w}$ is the Weinberg angle.

7) $N_{4} \rightarrow \nu_{\ell_{1}} \nu \bar{\nu}$ where $\nu_{\ell_{1}}=\nu_{e}, \nu_{\mu}, \nu_{\tau}$. This decay mode has neutral current interactions only as shown in Fig. 26. Using the massless approximation for the neutrinos as described above the decay width has a simple form given by

$$
\Gamma^{\nu_{\ell_{1}} \nu \nu} \equiv \sum_{\ell_{2}=e}^{\tau} \Gamma\left(N_{4} \rightarrow \nu_{\ell_{1}} \nu_{\ell_{2}} \overline{\nu_{\ell_{2}}}\right)=\frac{G_{F}^{2}}{96 \pi^{3}}\left|V_{\ell_{1} 4}\right|^{2} m_{4}^{5}
$$

All the decay modes listed above contribute to the total decay width of the heavy Majorana neutrino which is given by:

$$
\begin{aligned}
\Gamma_{N_{4}} & =\sum_{\ell, P} \Gamma^{\nu_{\ell} P}+\sum_{\ell, V} \Gamma^{\nu_{\ell} V}+\sum_{\ell, P} 2 \Gamma^{\ell P}+\sum_{\ell, V} 2 \Gamma^{\ell V} \\
& +\sum_{\ell_{1}, \ell_{2}\left(\ell_{1} \neq \ell_{2}\right)} 2 \Gamma^{\ell_{1} \ell_{2} \nu_{\ell_{2}}}+\sum_{\ell_{1}, \ell_{2}} \Gamma^{\nu_{\ell_{1}} \ell_{2} \ell_{2}}+\sum_{\nu_{\ell_{1}}} \Gamma^{\nu_{\ell_{1}} \nu \nu},
\end{aligned}
$$

where $\ell, \ell_{1}, \ell_{2}=e, \mu, \tau$. For a Majorana neutrino, the $\Delta L=0$ process $N_{4} \rightarrow \ell^{-} P^{+}$as well as its charge conjugate $|\Delta L|=2$ process $N_{4} \rightarrow \ell^{+} P^{-}$are possible and have the same width, $\Gamma^{\ell P}$. Hence the factor of 2 associated with the decay width of this mode in Eq. (C.9). Similarly, the $\Delta L=0$ and its charge conjugate $|\Delta L|=2$ process are possible for the decay modes $N_{4} \rightarrow \ell^{-} V^{+}$and $N_{4} \rightarrow \ell_{1}^{-} \ell_{2}^{+} \nu_{\ell_{2}}$ and hence have a factor of 2 associated with their width in Eq. (C.9).

As mentioned earlier, new channels open with increasing mass of the heavy neutrino. For the low energy $L V$ tau decays and rare meson decays we consider, the mass of the heavy neutrino is in the range $140 \mathrm{MeV} \lesssim \mathrm{m}_{4} \lesssim 5278 \mathrm{MeV}$. For this mass range we list all the possible decay channels for $N_{4}$ in Table 6. The mass and decay constants of pseudoscalar and vector mesons used in the calculation of partial widths given in Eqs. C.1 C.8) are listed in Table 7 in Appendix E.

\section{Lepton-number violating tau decay}

The decay amplitude for lepton number violating tau decays can be separated into leptonic and hadronic parts,

$$
i \mathcal{M}=\left(\mathcal{M}_{\text {lep }}\right)_{\mu \nu}\left(\mathcal{M}_{\text {had }}\right)^{\mu \nu}
$$

For the tree level amplitude, the hadronic part can be expressed in terms of the decay constants of the mesons in a model independent way. The box diagram includes hadronic matrix elements which cannot be simplified in terms of decay constants and needs to be evaluated in a model dependent way. We expect the tree level amplitude to dominate and do not include the box diagram. It has been argued that in certain cases for rare meson decays sub-leading contributions may be appreciable [113, 20]. Even in such a scenario the difference will not be important at the current level of sensitivities and we include the more conservative limit from tree level diagrams only. The tau decays and the rare meson decays are crossed versions of each other and the above arguments are true for both. 
Table 6: Decay modes of heavy Majorana neutrino based on its mass $m_{4}$.

\begin{tabular}{|c|c|c|c|}
\hline $\begin{array}{l}\text { Mass of heavy } \\
\text { neutrino }(\mathrm{MeV})\end{array}$ & $\begin{array}{l}\text { Decay mode of } \\
\text { heavy neutrino }\end{array}$ & $\begin{array}{l}\text { Mass of heavy } \\
\text { neutrino }(\mathrm{MeV})\end{array}$ & $\begin{array}{l}\text { Decay mode of } \\
\text { heavy neutrino }\end{array}$ \\
\hline$\gtrsim \sum_{m} \nu_{m}=10^{-6}$ & 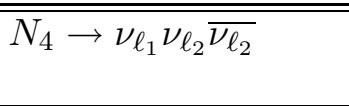 & $>m_{\mu}+m_{\tau}=1880$ & $\begin{array}{l}N_{4} \rightarrow \mu^{-} \tau^{+} \nu_{\tau}+c . c \\
N_{4} \rightarrow \tau^{-} \mu^{+} \nu_{\mu}+c . c\end{array}$ \\
\hline$>2 m_{e}=1.02$ & $N_{4} \rightarrow \nu_{\ell} e^{-} e^{+}$ & $>m_{\tau}+m_{\pi}=1920$ & $N_{4} \rightarrow \tau^{-} \pi^{+}+c . c$ \\
\hline$>m_{e}+m_{\mu}=106$ & $\begin{array}{l}N_{4} \rightarrow e^{-} \mu^{+} \nu_{m}+c . c \\
N_{4} \rightarrow \mu^{-} e^{+} \nu_{e}+c . c\end{array}$ & $>m_{e}+m_{D_{s}}=1970$ & $N_{4} \rightarrow e^{-} D_{s}^{+}+c . c$ \\
\hline$>m_{\pi^{0}}=135$ & $N_{4} \rightarrow \nu_{\ell} \pi^{0}$ & $>m_{\mu}+m_{D}=1980$ & $N_{4} \rightarrow \mu^{-} D^{+}+c . c$ \\
\hline$>m_{e}+m_{\pi}=140$ & $N_{4} \rightarrow e^{-} \pi^{+}+c . c$ & $>m_{D^{* 0}}=2010$ & $N_{4} \rightarrow \nu_{\ell} D^{* 0}$ \\
\hline$>2 m_{\mu}=211$ & $N_{4} \rightarrow \nu_{\ell} \mu^{-} \mu^{+}$ & $>m_{\bar{D}^{* 0}}=2010$ & $N_{4} \rightarrow \nu_{\ell} \bar{D}^{* 0}$ \\
\hline$>m_{\mu}+m_{\pi}=245$ & $N_{4} \rightarrow \mu^{-} \pi^{+}+c . c$ & $>m_{e}+m_{D^{*}}=2010$ & $N_{4} \rightarrow e^{-} D^{*^{+}}+c . c$ \\
\hline$>m_{e}+m_{K}=494$ & $N_{4} \rightarrow e^{-} K^{+}+c . c$ & $>m_{\mu}+m_{D_{s}}=2070$ & $N_{4} \rightarrow \mu^{-} D_{s}^{+}+c . c$ \\
\hline$>m_{\eta}=548$ & $N_{4} \rightarrow \nu_{\ell} \eta$ & $>m_{e}+m_{D_{s}^{*}}=2110$ & $N_{4} \rightarrow e^{-} D_{s}^{*+}+c . c$ \\
\hline$>m_{\mu}+m_{K}=599$ & $N_{4} \rightarrow \mu^{-} K^{+}+c . c$ & $>m_{\mu}+m_{D^{*}}=2120$ & $N_{4} \rightarrow \mu^{-} D^{*+}+c . c$ \\
\hline$>m_{\rho^{0}}=776$ & $N_{4} \rightarrow \nu_{\ell} \rho^{0}$ & $>m_{\mu}+m_{D_{s}^{*}}=2220$ & $N_{4} \rightarrow \mu^{-} D_{s}^{*+}+c . c$ \\
\hline$>m_{e}+m_{\rho}=776$ & $N_{4} \rightarrow e^{-} \rho^{+}+c . c$ & $>m_{\tau}+m_{K}=2270$ & $N_{4} \rightarrow \tau^{-} K^{+}+c . c$ \\
\hline$>m_{\omega}=783$ & $N_{4} \rightarrow \nu_{\ell} \omega$ & $>m_{\tau}+m_{\rho}=2550$ & $N_{4} \rightarrow \tau^{-} \rho^{+}+c . c$ \\
\hline$>m_{\mu}+m_{\rho}=882$ & $N_{4} \rightarrow \mu^{-} \rho^{+}+c . c$ & $>m_{\tau}+m_{K}^{*}=2670$ & $N_{4} \rightarrow \tau^{-} K^{*+}+c . c$ \\
\hline$>m_{e}+m_{K^{*}}=892$ & $N_{4} \rightarrow e^{-} K^{*+}+$ c.c & $>m_{\eta_{c}}=2980$ & $N_{4} \rightarrow \nu_{\ell} \eta_{c}$ \\
\hline$>m_{K^{* 0}}=896$ & $N_{4} \rightarrow \nu_{\ell} K^{* 0}$ & $>m_{J / \psi}=3100$ & $N_{4} \rightarrow \nu_{\ell} J / \psi$ \\
\hline$>m_{\bar{K}^{* 0}}=896$ & $N_{4} \rightarrow \nu_{\ell} \bar{K}^{* 0}$ & $>2 m_{\tau}=3550$ & $N_{4} \rightarrow \nu_{\ell} \tau^{-} \tau^{+}$ \\
\hline$>m_{\eta^{\prime}}=958$ & $N_{4} \rightarrow \nu_{\ell} \eta^{\prime}$ & $>m_{\tau}+m_{D}=3650$ & $N_{4} \rightarrow \tau^{-} D^{+}+c . c$ \\
\hline$>m_{\mu}+m_{K^{*}}=997$ & $N_{4} \rightarrow \mu^{-} K^{*+}+c . c$ & $>m_{\tau}+m_{D_{s}}=3750$ & $N_{4} \rightarrow \tau^{-} D_{s}^{+}+c . c$ \\
\hline$>m_{\phi}=1019$ & $N_{4} \rightarrow \nu_{\ell} \phi$ & $>m_{\tau}+m_{D^{*}}=3790$ & $N_{4} \rightarrow \tau^{-} D^{*+}+c . c$ \\
\hline$>m_{e}+m_{\tau}=1780$ & $\begin{array}{l}N_{4} \rightarrow e^{-} \tau^{+} \nu_{\tau}+c . c \\
N_{4} \rightarrow \tau^{-} e^{+} \nu_{e}+c . c\end{array}$ & $>m_{\tau}+m_{D_{s}^{*}}=3890$ & $N_{4} \rightarrow \tau^{-} D_{s}^{*+}+c . c$ \\
\hline$>m_{e}+m_{D}=1870$ & $N_{4} \rightarrow e^{-} D^{+}+c . c$ & & \\
\hline
\end{tabular}

The leptonic part of the subprocess $\tau^{-} \rightarrow \ell^{+} W^{-*} W^{-*}$ is obtained by crossing the amplitude in (B.6)

$$
\mathcal{M}_{l e p}^{\mu \nu}=\frac{g^{2}}{2} V_{\tau 4}^{*} V_{\ell 4}^{*} \overline{v_{\tau}} \frac{m_{4}}{q^{2}-m_{4}^{2}+i \Gamma_{N_{4}} m_{4}} \gamma^{\mu} \gamma^{\nu} P_{R} v_{\ell}
$$

Combining the hadronic and leptonic parts, the decay amplitude for

$$
\tau^{-}\left(p_{1}\right) \rightarrow \ell^{+}\left(p_{2}\right) M_{1}^{-}\left(q_{1}\right) M_{2}^{-}\left(q_{2}\right)
$$

is given by

$$
\begin{aligned}
i \mathcal{M} & =\left(\mathcal{M}_{\text {lep }}\right)_{\mu \nu} \mathcal{M}_{M_{1}}^{\mu} \mathcal{M}_{M_{2}}^{\nu}+\left(M_{1} \leftrightarrow M_{2}\right) \\
& =2 G_{F}^{2} V_{M_{1}}^{C K M} V_{M_{2}}^{C K M} f_{M_{1}} f_{M_{2}} V_{\tau 4}^{*} V_{\ell 4}^{*} m_{4}\left[\frac{\overline{v_{\tau}} q_{1} q_{2} P_{R} v_{\ell}}{\left(p_{1}-q_{1}\right)^{2}-m_{4}^{2}+i \Gamma_{N_{4}} m_{4}}\right]
\end{aligned}
$$




$$
\begin{aligned}
& +2 G_{F}^{2} V_{M_{1}}^{C K M} V_{M_{2}}^{C K M} f_{M_{1}} f_{M_{2}} V_{\tau 4}^{*} V_{\ell 4}^{*} m_{4}\left[\frac{\overline{v_{\tau}} \not q_{2} \not q_{1} P_{R} v_{\ell}}{\left(p_{1}-q_{2}\right)^{2}-m_{4}^{2}+i \Gamma_{N_{4}} m_{4}}\right], \\
& =\mathcal{M}_{1}+\mathcal{M}_{2}
\end{aligned}
$$

where $V_{M_{i}}^{C K M}$ are the quark flavor-mixing matrix elements for the mesons and $f_{M_{i}}$ are meson decay constants. Then the functions, $f_{1}$ and $f_{2}$ defined in Eq. (B.8) are given by

$$
\begin{aligned}
f_{1} & =\left(\frac{F_{\tau} A}{a_{1}^{2}+b^{2}}\right)\left[\frac{\left(a_{2}^{2}+b^{2}\right) A+\left(a_{1} a_{2}+b^{2}\right) C}{\left(a_{2}^{2}+b^{2}\right) A+\left(a_{1}^{2}+b^{2}\right) B}+\left(q_{1} \leftrightarrow q_{2}\right)\right], \\
f_{2} & =f_{1}\left(q_{1} \leftrightarrow q_{2}\right), \\
A\left(p_{i}, q_{j}\right) & =8\left(p_{1} \cdot q_{1}\right)\left(p_{2} \cdot q_{2}\right)\left(q_{1} \cdot q_{2}\right)-4 m_{M_{1}}^{2}\left(p_{1} \cdot q_{2}\right)\left(p_{2} \cdot q_{2}\right) \\
& -4 m_{M_{2}}^{2}\left(p_{1} \cdot q_{1}\right)\left(p_{2} \cdot q_{1}\right)+2 m_{M_{1}}^{2} m_{M_{2}}^{2}\left(p_{1} \cdot p_{2}\right), \\
B\left(p_{i}, q_{j}\right) & =A\left(q_{1} \leftrightarrow q_{2}\right), \\
C\left(p_{i}, q_{j}\right) & =4\left(p_{1} \cdot p_{2}\right)\left(q_{1} \cdot q_{2}\right)^{2}-A\left(p_{i}, q_{j}\right), \\
D\left(p_{i}, q_{j}\right) & =C\left(q_{1} \leftrightarrow q_{2}\right), \\
F_{\tau} & =4 G_{F}^{4} f_{M_{1}}^{2} f_{M_{2}}^{2}\left|V_{M_{1}}^{C K M} V_{M_{2}}^{C K M}\right|^{2}\left|V_{\tau 4} V_{\ell 4}\right|^{2} m_{4}^{2}, \\
a_{1,2}\left(p_{i}, q_{j}\right) & =\left(p_{1}-q_{1,2}\right)^{2}-m_{4}^{2} ; \quad b=\Gamma_{N_{4}} m_{4} .
\end{aligned}
$$

The decay width for the $L V$ tau decay is then given by

$$
\begin{aligned}
\Gamma_{L V}^{\tau} & =\left(1-\frac{1}{2} \delta_{M_{1} M_{2}}\right) \frac{1}{128 \pi^{5} m_{\tau}}\left[\int f_{1} d P S_{31}+\int f_{2} d P S_{32}\right], \\
d P S_{31} & =\frac{\pi^{2}}{4 m_{\tau}^{2}} \lambda^{\frac{1}{2}}\left(m_{\tau}^{2}, m_{M_{1}}^{2}, m_{c 1}^{2}\right) \lambda^{\frac{1}{2}}\left(m_{c 1}^{2}, m_{\ell}^{2}, m_{M_{2}}^{2}\right) \frac{d m_{c 1}^{2}}{m_{c 1}^{2}} d y_{1} d y_{2} d y_{3} d y_{4}, \\
d P S_{32} & =d P S_{31}\left(q_{1} \leftrightarrow q_{2}\right),
\end{aligned}
$$

where $d P S_{31}$ and $d P S_{32}$ are the phase space factors obtained by conveniently clustering two different sets of particles to enable applying the narrow-width approximation easily. $y_{1}$ to $y_{4}$ are rescaled angular variables with integration limits $0 \leq y_{i} \leq 1$. As seen in Sec. 3.1.1, the width of the heavy neutrino is very small compared to the mass and hence we can apply the narrow-width approximation.

$$
\left.\int \frac{d m_{c_{i}}^{2}}{\left(m_{c_{i}}^{2}-m_{4}^{2}\right)^{2}+\Gamma_{N_{4}}^{2} m_{4}^{2}}\right|_{\Gamma_{N_{4}} \rightarrow 0}=\int \delta\left(m_{c_{i}}^{2}-m_{4}^{2}\right) d m_{c_{i}}^{2} \frac{\pi}{\Gamma_{N_{4}} m_{4}}
$$

Applying the narrow-width approximation as described above and integrating over the $\delta$-function we get

$$
\begin{aligned}
\int f_{1} d P S_{31}= & \int\left(\frac{F_{\tau} A \pi^{3}}{4 m_{\tau}^{2} m_{4}^{3} \Gamma_{N_{4}}}\left[\frac{\left(a_{2}^{2}+b^{2}\right) A+b^{2}(B+C+D)}{\left(a_{2}^{2}+b^{2}\right) A+b^{2} B}\right]\right. \\
& \left.\lambda^{\frac{1}{2}}\left(m_{\tau}^{2}, m_{M_{1}}^{2}, m_{4}^{2}\right) \lambda^{\frac{1}{2}}\left(m_{4}^{2}, m_{\ell}^{2}, m_{M_{2}}^{2}\right)\right) d y_{1} d y_{2} d y_{3} d y_{4}, \\
\int f_{2} d P S_{32}= & \int f_{1} d P S_{31}\left(q_{1} \leftrightarrow q_{2}\right)
\end{aligned}
$$


Now, we can find the decay rate from Eq. (D.15). Normalized to the $\tau$ decay width $\Gamma_{\tau}=G_{F}^{2} m_{\tau}^{5} / 192 \pi^{3}$, the corresponding branching fraction is $\mathrm{Br}=\Gamma_{L V}^{\tau} / \Gamma_{\tau}$. The masses and decay constants of mesons are listed in Table 7 . The CKM matrix elements and $\tau$ mass are taken from the Particle Data Group (PDG) 99:

$$
m_{\tau}=1777 \mathrm{MeV},\left|V_{u d}\right|=0.9738,\left|V_{u s}\right|=0.2200 \text {. }
$$

\section{E. Rare meson decay}

The rare meson decays

$$
M_{1}^{+}\left(q_{1}\right) \rightarrow \ell^{+}\left(p_{1}\right) \ell^{+}\left(p_{2}\right) M_{2}^{-}\left(q_{2}\right)
$$

have the same Feynman diagrams as tau decay. The meson $M_{2}$ can be a pseudoscalar or vector meson. The decay amplitude when $M_{2}$ is a pseudoscalar meson is given by

$$
\begin{aligned}
i \mathcal{M}^{P} & =2 G_{F}^{2} V_{M_{1}}^{C K M} V_{M_{2}}^{C K M} f_{M_{1}} f_{M_{2}} V_{\ell_{1} 4} V_{\ell_{2} 4} m_{4}\left[\frac{\overline{u_{\ell_{1}}} q_{1} q_{2} P_{R} v_{\ell_{2}}}{\left(q_{1}-p_{1}\right)^{2}-m_{4}^{2}+i \Gamma_{N_{4}} m_{4}}\right] \\
& +2 G_{F}^{2} V_{M_{1}}^{C K M} V_{M_{2}}^{C K M} f_{M_{1}} f_{M_{2}} V_{\ell_{1} 4} V_{\ell_{2} 4} m_{4}\left[\frac{\overline{u_{\ell_{1}}} \not q_{2} \not q_{1} P_{R} v_{\ell_{2}}}{\left(q_{1}-p_{2}\right)^{2}-m_{4}{ }^{2}+i \Gamma_{N_{4}} m_{4}}\right] \\
& =\mathcal{M}_{1}^{P}+\mathcal{M}_{2}^{P} .
\end{aligned}
$$

Next we consider the case where $M_{2}$ is a vector meson. The decay amplitude is given by

$$
\begin{aligned}
i \mathcal{M}^{V} & =2 G_{F}^{2} V_{M_{1}}^{C K M} V_{M_{2}}^{C K M} f_{M_{1}} f_{M_{2}} V_{\ell_{1} 4} V_{\ell_{2} 4} m_{4} m_{M_{2}}\left[\frac{\overline{u_{\ell_{1}}} q_{1} \not k^{\lambda}\left(q_{2}\right) P_{R} v_{\ell_{2}}}{\left(q_{1}-p_{1}\right)^{2}-m_{4}{ }^{2}+i \Gamma_{N_{4}} m_{4}}\right] \\
& +2 G_{F}^{2} V_{M_{1}}^{C K M} V_{M_{2}}^{C K M} f_{M_{1}} f_{M_{2}} V_{\ell_{1} 4} V_{\ell_{2} 4} m_{4} m_{M_{2}}\left[\frac{\overline{u_{\ell_{1}}} \not k^{\lambda}\left(q_{2}\right) \not q_{1} P_{R} v_{\ell_{2}}}{\left(q_{1}-p_{2}\right)^{2}-m_{4}{ }^{2}+i \Gamma_{N_{4}} m_{4}}\right] \\
& =\mathcal{M}_{1}^{V}+\mathcal{M}_{2}^{V} .
\end{aligned}
$$

Similar to tau decay, we define the functions $f_{1}^{P}, f_{2}^{P}$ and $f_{1}^{V}, f_{2}^{V}$ for the pseudoscalar and vector mesons respectively as given in Eq. (B.8). $f_{i}^{P}$ and $f_{i}^{V}$ turn out to have the same form and are given below.

$$
\begin{aligned}
f_{1}^{P(V)} & =\left(\frac{F_{M} A^{P(V)}}{a_{1}^{2}+b^{2}}\right)\left[\frac{\left(a_{2}^{2}+b^{2}\right) A^{P(V)}+\left(a_{1} a_{2}+b^{2}\right) C^{P(V)}}{\left(a_{2}^{2}+b^{2}\right) A^{P(V)}+\left(a_{1}^{2}+b^{2}\right) B^{P(V)}}+\left(p_{1} \leftrightarrow p_{2}\right)\right], \\
f_{2}^{P(V)} & =f_{1}^{P(V)}\left(p_{1} \leftrightarrow p_{2}\right) \\
A^{P}\left(p_{i}, q_{j}\right) & =8\left(p_{1} \cdot q_{1}\right)\left(p_{2} \cdot q_{2}\right)\left(q_{1} \cdot q_{2}\right)-4 m_{M_{1}}^{2}\left(p_{1} \cdot q_{2}\right)\left(p_{2} \cdot q_{2}\right) \\
& -4 m_{M_{2}}^{2}\left(p_{1} \cdot q_{1}\right)\left(p_{2} \cdot q_{1}\right)+2 m_{M_{1}}^{2} m_{M_{2}}^{2}\left(p_{1} \cdot p_{2}\right) \\
A^{V}\left(p_{i}, q_{j}\right) & =8\left(p_{1} \cdot q_{1}\right)\left(p_{2} \cdot q_{2}\right)\left(q_{1} \cdot q_{2}\right)-4 m_{M_{1}}^{2}\left(p_{1} \cdot q_{2}\right)\left(p_{2} \cdot q_{2}\right) \\
& +4 m_{M_{2}}^{2}\left(p_{1} \cdot q_{1}\right)\left(p_{2} \cdot q_{1}\right)-2 m_{M_{1}}^{2} m_{M_{2}}^{2}\left(p_{1} \cdot p_{2}\right) \\
B^{P(V)}\left(p_{i}, q_{j}\right) & =A^{P(V)}\left(p_{1} \leftrightarrow p_{2}\right) \\
C^{P}\left(p_{i}, q_{j}\right) & =4\left(p_{1} \cdot p_{2}\right)\left(q_{1} \cdot q_{2}\right)^{2}-A^{P}\left(p_{i}, q_{j}\right) \\
C^{V}\left(p_{i}, q_{j}\right) & =4\left(p_{1} \cdot p_{2}\right)\left(q_{1} \cdot q_{2}\right)^{2}-4 m_{M_{1}}^{2} m_{M_{2}}^{2}\left(p_{1} \cdot p_{2}\right)-A^{V}\left(p_{i}, q_{j}\right)
\end{aligned}
$$




$$
\begin{aligned}
D^{P(V)}\left(p_{i}, q_{j}\right) & =C^{P(V)}\left(p_{1} \leftrightarrow p_{2}\right), \\
F_{M} & =4 G_{F}^{4} f_{M_{1}}^{2} f_{M_{2}}^{2}\left|V_{M_{1}}^{C K M} V_{M_{2}}^{C K M}\right|^{2}\left|V_{\ell_{1} 4} V_{\ell_{2} 4}\right|^{2} m_{4}^{2}, \\
a_{1,2}\left(p_{i}, q_{j}\right) & =\left(q_{1}-p_{1,2}\right)^{2}-m_{4}^{2} ; \quad b=\Gamma_{N_{4}} m_{4} .
\end{aligned}
$$

The decay rate for $L V$ rare meson decay is then given by

$$
\begin{aligned}
\Gamma_{L V}^{M_{1}} & =\left(1-\frac{1}{2} \delta_{\ell_{1} \ell_{2}}\right) \frac{1}{64 \pi^{5} m_{M_{1}}}\left[\int f_{1}^{P(V)} d P S_{31}+\int f_{2}^{P(V)} d P S_{32}\right], \\
d P S_{31} & =\frac{\pi^{2}}{4 m_{M_{1}}^{2}} \lambda^{\frac{1}{2}}\left(m_{M_{1}}^{2}, m_{\ell_{1}}^{2}, m_{c 1}^{2}\right) \lambda^{\frac{1}{2}}\left(m_{c 1}^{2}, m_{\ell_{2}}^{2}, m_{M_{2}}^{2}\right) \frac{d m_{c 1}^{2}}{m_{c 1}^{2}} d y_{1} d y_{2} d y_{3} d y_{4}, \\
d P S_{32} & =d P S_{31}\left(p_{1} \leftrightarrow p_{2}\right),
\end{aligned}
$$

where $d P S_{31}$ and $d P S_{32}$ are the phase space factors obtained by conveniently clustering two different sets of particles to enable applying the narrow-width approximation easily. $y_{1}$ to $y_{4}$ are rescaled angular variables with integration limits $0 \leq y_{i} \leq 1$. The width of the heavy neutrino is very small compared to the mass and hence we can apply the narrow-width approximation.

$$
\left.\int \frac{d m_{c_{i}}^{2}}{\left(m_{c_{i}}^{2}-m_{4}^{2}\right)^{2}+\Gamma_{N_{4}}^{2} m_{4}^{2}}\right|_{\Gamma_{N_{4}} \rightarrow 0}=\int \delta\left(m_{c_{i}}^{2}-m_{4}^{2}\right) d m_{c_{i}}^{2} \frac{\pi}{\Gamma_{N_{4}} m_{4}}
$$

Applying the narrow-width approximation as described above and integrating over the $\delta$-function we get

$$
\begin{aligned}
\int f_{1}^{P(V)} d P S_{31}= & \int\left(\frac{F_{M} A^{P(V)} \pi^{3}}{4 m_{M 1}^{2} m_{4}^{3} \Gamma_{N_{4}}}\left[\frac{\left(a_{2}^{2}+b^{2}\right) A^{P(V)}+b^{2}\left(B^{P(V)}+C^{P(V)}+D^{P(V)}\right)}{\left(a_{2}^{2}+b^{2}\right) A^{P(V)}+b^{2} B^{P(V)}}\right]\right. \\
& \left.\lambda^{\frac{1}{2}}\left(m_{M_{1}}^{2}, m_{\ell_{1}}^{2}, m_{4}^{2}\right) \lambda^{\frac{1}{2}}\left(m_{4}^{2}, m_{\ell_{2}}^{2}, m_{M_{2}}^{2}\right)\right) d y_{1} d y_{2} d y_{3} d y_{4} \\
\int f_{2}^{P(V)} d P S_{32}= & \int f_{1}^{P(V)} d P S_{31}\left(p_{1} \leftrightarrow p_{2}\right),
\end{aligned}
$$

Now, we can find the decay rate from Eq. (E.13). The branching fraction is then given by $\mathrm{Br}=\tau_{M_{1}} \Gamma_{L V}^{M_{1}}$.

The CKM matrix elements and the lifetimes of mesons used in our calculations are taken from PDG [99] and are listed below.

$$
\begin{aligned}
& \left|V_{u b}\right|=0.00367,\left|V_{c d}\right|=0.224,\left|V_{c s}\right|=0.996 ; \\
& \tau_{K}=1.2384 \times 10^{-8} \mathrm{~s}, \tau_{D}=1.040 \times 10^{-12} \mathrm{~s}, \tau_{D_{s}}=4.9 \times 10^{-13} \mathrm{~s}, \tau_{B}=1.671 \times 10^{-12} \mathrm{~s} .
\end{aligned}
$$

The mass and decay constants of pseudoscalar and vector mesons used in our calculations are listed in Table 7 . 
Table 7: Mass and decay constants of pseudoscalar and vector mesons used.

\begin{tabular}{|c|c|c|c|c|c|}
\hline $\begin{array}{c}\text { Pseudoscalar } \\
\text { Meson }\end{array}$ & $\begin{array}{c}\text { Mass } \\
(\mathrm{MeV})[\mathbf{9 9}]\end{array}$ & $\begin{array}{c}\text { Decay Constant } \\
(\mathrm{MeV})[99]\end{array}$ & $\begin{array}{c}\text { Vector } \\
\text { Meson }\end{array}$ & $\begin{array}{c}\text { Mass } \\
(\mathrm{MeV})[99]\end{array}$ & $\begin{array}{c}\text { Decay Constant } \\
(\mathrm{MeV})[114]\end{array}$ \\
\hline$\pi^{ \pm}$ & 139.6 & 130.7 & $\rho^{ \pm}$ & 775.8 & 220 \\
\hline$K^{ \pm}$ & 493.7 & 159.8 & $K^{* \pm}$ & 891.66 & 217 \\
\hline$D^{ \pm}$ & 1869.4 & $222.6[115]$ & $D^{*}$ & 2010 & 310 \\
\hline$D_{s}^{ \pm}$ & 1968.3 & 266 & $D_{s}^{* \pm}$ & 2112.1 & 315 \\
\hline$B^{ \pm}$ & 5279 & $190[116]$ & $\omega$ & 782.59 & 195 \\
\hline$\pi^{0}$ & 135 & 130 & $K^{* 0}, \bar{K}^{* 0}$ & 896.10 & 217 \\
\hline$\eta$ & 547.8 & $164.7[117]$ & $\phi$ & 1019.456 & 229 \\
\hline$\eta^{\prime}$ & 957.8 & $152.9[117]$ & $D^{* 0}, \bar{D}^{* 0}$ & 2006.7 & 310 \\
\hline$\eta_{c}$ & 2979.6 & $335.0[118]$ & $J / \psi$ & 3096.916 & $459[119]$ \\
\hline
\end{tabular}

\section{References}

[1] For earlier comprehensive discussions of neutrino physics see e.g., Massive Neutrinos in Physics and Astrophysics by R. N. Mohapatra and P. B. Pal (World Scientific 2004); Physics of Neutrinos and Applications to Astrophysics by M. Fukugita and T. Yanagida (Springer-Verlag 2003). For recent reviews, see e.g., V. Barger, D. Marfatia, and K. Whisnant, Int. J. Mod. Phys. E12, 569 (2003); B. Kayser, p. 145 in PDG in Phys. Lett. B592, 1 (2004); M. C. Gonzalez-Garcia and M. Maltoni, arXiv:0704.1800 [hep-ph]; R. N. Mohapatra and A. Y. Smirnov, Ann. Rev. Nucl. Part. Sci. 56 (2006) 569; A. Strumia and F. Vissani, arXiv:hep-ph/0606054.

[2] P. Minkowski, Phys. Lett. B67, 421 (1977); T. Yanagida, in Proc. of the Workshop on Grand Unified Theory and Baryon Number of the Universe, KEK, Japan, 1979; M. Gell-Mann, P. Ramond and R. Slansky in Sanibel Symposium, February 1979, CALT-68-709 [retroprint arXiv:hep-ph/9809459], and in Supergravity, eds. D. Freedman et al. (North Holland, Amsterdam, 1979); S. L. Glashow in Quarks and Leptons, Cargese, eds. M. Levy et al. (Plenum, 1980, New York), p. 707; R. N. Mohapatra and G. Senjanovic, Phys. Rev. Lett. 44, $912(1980)$.

[3] J. C. Pati and A. Salam, Phys. Rev. D10, 275 (1974); R. N. Mohapatra and J. C. Pati, Phys. Rev. D11, 566, 2558 (1975); G. Senjanovic and R. N. Mohapatra, Phys. Rev. D12, 1502 (1975).

[4] J. A. Harvey, P. Ramond and D. B. Reiss, Nucl. Phys. B199, 223 (1982); S. Dimopoulos, L. J. Hall and S. Raby, Phys. Rev. Lett. 68, 1984 (1992); L. J. Hall and S. Raby, Phys. Rev. D51, 6524 (1995) [arXiv:hep-ph/9501298].

[5] I. Dorsner and P. Fileviez Pérez, Nucl. Phys. B 723 (2005) 53 [arXiv:hep-ph/0504276]; see also: I. Dorsner, P. Fileviez Pérez and R. Gonzalez Felipe, Nucl. Phys. B 747 (2006) 312 [arXiv:hep-ph/0512068]; P. Fileviez Pérez, AIP Conf. Proc. 903 (2006) 385 [arXiv:hep-ph/0606279]; I. Dorsner, P. Fileviez Pérez and G. Rodrigo, Phys. Rev. D 75 (2007) 125007 [arXiv:hep-ph/0607208]. 
[6] A. Zee, Phys. Lett. B93, 389 (1980) [Erratum - ibid. B95, 461 (1980)]; Phys. Lett B161, 141 (1985).

[7] E. Ma and U. Sarkar, Phys. Rev. Lett. 80, 5716 (1998) [arXiv:hep-ph/9802445]; E. Ma and G. Rajasekaran, Phys. Rev. D64, 113012 (2001) [arXiv:hep-ph/0106291]; E. Ma, Mod. Phys. Lett. A17, 289 (2002) [arXiv:hep-ph/0201225]; K. S. Babu, E. Ma and J. W. Valle, Phys. Lett. B552, 207 (2003) [arXiv:hep-ph/0206292]; E. Ma, Mod. Phys. Lett. A17, 2361 (2002) [arXiv:hep-ph/0211393].

[8] C. S. Aulakh and R. N. Mohapatra, Phys. Lett. B119, 136 (1982); L. J. Hall and M. Suzuki, Nucl. Phys. B231, 419 (1984); G. G. Ross and J. W. F. Valle, Phys. Lett. B151, 375 (1985); J. Ellis, G. Gelmini, C. Jarlskog, G. G. Ross and J. W. F. Valle, Phys. Lett. B150, 142 (1985); S. Dawson, Nucl. Phys. B261, 297 (1985); M. Drees, S. Pakvasa, X. Tata and T. ter. Veldhuis, Phys. Rev. D57, R5335 (1998) [arXiv:hep-ph/9712392]; E. J. Chun, S. K. Kang, C. W. Kim and U. W. Lee, Nucl. Phys. B544, 89 (1999) [arXiv:hep-ph/9807327]; V. Barger, T. Han, S. Hesselbach and D. Marfatia, Phys. Lett. B538, 346 (2002) [arXiv:hep-ph/0108261]; for a recent review see R. Barbieri et al., Phys. Rept. 420, 1 (2005) [arXiv:hep-ph/0406039]; V. Barger, P. F. Perez and S. Spinner, arXiv:0812.3661 [hep-ph] .

[9] N. Arkani-Hamed, S. Dimopoulos, G. Dvali and J. March-Russell, Phys. Rev. D65, 024032 (2002) [arXiv:hep-ph/9811448]; Y. Grossman and M. Neubert, Phys. Lett. B 474, 361 (2000) [arXiv:hep-ph/9912408]; K. R. Dienes and I. Sarcevic, Phys. Lett. B500, 133 (2001) [arXiv:hep-ph/0008144]; S. J. Huber and Q. Shafi, Phys. Lett. B 544, 295 (2002) [arXiv:hep-ph/0205327]; M. C. Chen and H. B. Yu, arXiv:0804.2503 [hep-ph] : G. Perez and L. Randall, arXiv:0805.4652 [hep-ph] .

[10] W. Konetschny and W. Kummer, Phys. Lett. B 70 (1977) 433; J. Schechter and J. W. F. Valle, Phys. Rev. D 22 (1980) 2227; T. P. Cheng and L. F. Li, Phys. Rev. D 22 (1980) 2860; G. Lazarides, Q. Shafi and C. Wetterich, Nucl. Phys. B 181 (1981) 287; R. N. Mohapatra and G. Senjanović, Phys. Rev. D 23 (1981) 165.

[11] R. Foot, H. Lew, X. G. He and G. C. Joshi, Z. Phys. C 44 (1989) 441; E. Ma, Phys. Rev. Lett. 81 (1998) 1171 [arXiv:hep-ph/9805219]; B. Bajc and G. Senjanović, JHEP 0708 (2007) 014 [arXiv:hep-ph/0612029]; P. Fileviez Pérez, Phys. Lett. B 654 (2007) 189 [arXiv:hep-ph/0702287]; P. Fileviez Pérez, Phys. Rev. D 76 (2007) 071701 [arXiv:0705.3589 [hep-ph]]; P. Fileviez Perez, arXiv:0809.1202 [hep-ph].

[12] S. Weinberg, Phys. Rev. Lett. 43 (1979) 1566.

[13] K. S. Babu and C. N. Leung, Nucl. Phys. B619, 667 (2001) [arXiv:hep-ph/0106054].

[14] W. H. Furry, Phys. Rev. 56, 1184 (1939); for early reviews see, Primakoff and Rosen, Rep. Prog. Phys. 22, 121 (1959); Ann. Rev. Nucl. Part. Sci. 31, 145 (1981).

[15] M. Doi, T. Kotani and E. Takasugi, Prog. Theor. Phys. Suppl. 83, 1 (1985).

[16] For recent review see eg. S. R. Elliott and J. Engel, J. Physics. G30 R183 (2004) [arXiv:hep-ph/0405078].

[17] A. Ilakovac, B. A. Kniehl and A. Pilaftsis, Phys. Rev. D52, 3993 (1995) [arXiv:hep-ph/9503456]; A. Ilakovac and A. Pilaftsis, Nucl. Phys. B437, 491 (1995) 
[arXiv:hep-ph/9403398]; A. Ilakovac, Phys. Rev. D54, 5653 (1996)

[arXiv:hep-ph/9608218]; V. Gribanov, S. Kovalenko and I. Schmidt, Nucl. Phys. B 607, 355 (2001) [arXiv:hep-ph/0102155].

[18] A. Atre, V. Barger and T. Han, Phys. Rev. D71, 113014 (2005) [arXiv:hep-ph/0502163].

[19] J. N. Ng and A. N. Kamal, Phys. Rev. D 18, 3412 (1978); J. Abad, J. G. Esteve and A. F. Pacheco, Phys. Rev. D 30, 1488 (1984); C. Dib, V. Gribanov, S. Kovalenko and I. Schmidt, Phys. Lett. B 493, 82 (2000) [arXiv:hep-ph/0006277].

[20] A. Ali, A. V. Borisov and N. B. Zamorin, Eur. Phys. J. C21, 123 (2001) [arXiv:hep-ph/0104123].

[21] L. S. Littenberg and R. E. Shrock, Phys. Rev. D46, R892 (1992); C. Barbero, G. Lopez Castro and A. Mariano, Phys. Lett. B566, 98 (2003) [arXiv:nucl-th/0212083].

[22] C. S. Lim, E. Takasugi and M. Yoshimura, Prog. Theor. Phys. 113, 1367 (2005), [arXiv:hep-ph/0411139].

[23] SINDRUM II Collaboration, J. Kaulard et al., Phys. Lett. B422, 334 (1998); K. Zuber, arXiv:hep-ph/0008080; P. Domin, A. Faessler, S. Kovalenko and F. Simkovic, Phys. Rev. C70, 065501 (2004) [arXiv:nucl-th/0409033].

[24] J. H. Missimer, R. N. Mohapatra and N. C. Mukhopadhyay, Phys. Rev. D50, 2067 (1994); F. Simkovic, A. Faessler, S. Kovalenko and P. Domin, Phys. Rev. D66, 033005 (2002) [arXiv:hep-ph/0112271]; E. Takasugi, Nucl. Instrum. Meth. A503, 252 (2003); M. Aoki, Nucl. Instrum. Meth. A503, 258 (2003).

[25] T. G. Rizzo, Phys. Lett. B116, 23 (1982); C. A. Heusch and P. Minkowski, Nucl. Phys. B416, 3 (1994).

[26] M. Dittmar, A. Santamaria, M. C. Gonzalez-Garcia and J. W. F. Valle, Nucl. Phys. B 332, 1 (1990).

[27] M. Flanz, W. Rodejohann and K. Zuber, Phys. Lett. B473, 324 (2000), Erratum - ibid. B480, 418 (2000) [arXiv:hep-ph/9911298].

[28] M. Flanz, W. Rodejohann and K. Zuber, Eur. Phys. J. C16, 453 (2000) [arXiv:hep-ph/9907203]; W. Rodejohann and K. Zuber, Phys. Rev. D63, 054031 (2001) [arXiv:hep-ph/0011050].

[29] W.-Y. Keung and G. Senjanovic, Phys. Rev. Lett. 50, 1427 (1983); D. Dicus, D. Karatas, and P. Roy, Phys. Rev. D44, 2033 (1991); A. Datta, M. Guchait, and A. Pilaftsis, Phys. Rev. D50, 3195 (1994) [arXiv: hep-ph/9311257].

[30] F. M. L. Almeida, Y. A. Coutinho, J. A. M. Simoes and M. A. B. Vale, Phys. Rev. D62, 075004 (2000) [arXiv:hep-ph/0008201]; O. Panella, M. Cannoni, C. Carimalo, and Y. N. Srivastava, Phys. Rev. D65, 035005 (2002) [arXiv:hep-ph/0107308].

[31] T. Han and B. Zhang, Phys. Rev. Lett. 97, 171804 (2006) [arXiv:hep-ph/0604064].

[32] For a comparison for different colliders, see e.g., F. del Aguila, J. A. Aguilar-Saavedra and R. Pittau, J. Phys. Conf. Ser. 53, 506 (2006) [arXiv:hep-ph/0606198]. 
[33] F. del Aguila, J. A. Aguilar-Saavedra and R. Pittau, JHEP 0710, 047 (2007) [arXiv:hep-ph/0703261].

[34] W. Clarida, T. Yetkin, R. Vidal, W. Wu, Tao Han, H. Pi, and E. Yazgan, CMS Note 2008/054 (Dec. 2008) unpublished.

[35] S. Bar-Shalom, N. G. Deshpande, G. Eilam, J. Jiang and A. Soni, Phys. Lett. B643, 342 (2006) [arXiv:hep-ph/0608309]; Z.-G. Si and K. Wang, arXiv:0810.5266.

[36] D. Atwood, S. Bar-Shalom and A. Soni, Phys. Rev. D 76, 033004 (2007) [arXiv:hep-ph/0701005].

[37] S. Bar-Shalom, G. Eilam, T. Han and A. Soni, Phys. Rev. D77, 115019 (2008) [arXiv:0803.2835[hep-ph]].

[38] I. Dorsner and P. Fileviez Perez, JHEP 0706, 029 (2007) [arXiv:hep-ph/0612216]; B. Bajc, M. Nemevsek and G. Senjanović, Phys. Rev. D 76, 055011 (2007) [arXiv:hep-ph/0703080] .

[39] A. de Gouvea, J. Jenkins and N. Vasudevan, Phys. Rev. D 75, 013003 (2007) [arXiv:hep-ph/0608147]; A. de Gouvea, arXiv:0706.1732 [hep-ph].

[40] A. Pilaftsis and T. E. J. Underwood, Nucl. Phys. B 692, 303 (2004) [arXiv:hep-ph/0309342]; A. Pilaftsis and T. E. J. Underwood, Phys. Rev. D 72, 113001 (2005) [arXiv:hep-ph/0506107].

[41] T. Appelquist and R. Shrock, Phys. Rev. Lett. 90, 201801 (2003) [arXiv:hep-ph/0301108]; ibidem, Phys. Lett. B 548, 204 (2002) [arXiv:hep-ph/0204141]; ibidem, in Neutrino Factories and Superbeams, NuFact03, A.I.P. Conf. Proc, 721 (A.I.P., New York, 2004), p. 261; T. Appelquist, M. Piai and R. Shrock, Phys. Rev. D 69, 015002 (2004) [arXiv:hep-ph/0308061]; T. Appelquist, N. D. Christensen, M. Piai and R. Shrock, Phys. Rev. D 70, 093010 (2004) [arXiv:hep-ph/0409035].

[42] K. R. S. Balaji, A. Perez-Lorenzana and A. Y. Smirnov, Phys. Lett. B 509, 111 (2001) [arXiv:hep-ph/0101005]; A. Y. Smirnov and R. Zukanovich Funchal, Phys. Rev. D 74, 013001 (2006) [arXiv:hep-ph/0603009].

[43] M. Viel, J. Lesgourgues, M. G. Haehnelt, S. Matarrese and A. Riotto, Phys. Rev. D 71, 063534 (2005) [arXiv:astro-ph/0501562]; M. Viel, J. Lesgourgues, M. G. Haehnelt, S. Matarrese and A. Riotto, Phys. Rev. Lett. 97, 071301 (2006) [arXiv:astro-ph/0605706].

[44] G. M. Fuller, A. Kusenko and K. Petraki, arXiv:0806 .4273 [astro-ph].

[45] S. Dodelson and L. M. Widrow, Phys. Rev. Lett. 72, 17 (1994) [arXiv:hep-ph/9303287]; X. D. Shi and G. M. Fuller, Phys. Rev. Lett. 82 [arXiv:astro-ph/9810076], 2832 (1999); A. D. Dolgov and S. H. Hansen, Astropart. Phys. 16, 339 (2002).

[46] K. Abazajian, G. M. Fuller and M. Patel, Phys. Rev. D 64, 023501 (2001) [arXiv:astro-ph/0101524].

[47] G. Gelmini, S. Palomares-Ruiz and S. Pascoli, Phys. Rev. Lett. 93, 081302 (2004) [arXiv:astro-ph/0403323].

[48] A. D. Dolgov, Phys. Rept. 370, 333 (2002) [arXiv:hep-ph/0202122]. 
[49] A. Kusenko and G. Segre, Phys. Lett. B396, 197 (1997) [arXiv:hep-ph/9701311]; G. M. Fuller, A. Kusenko, I. Mocioiu and S. Pascoli, Phys. Rev, D68, 103002 (2003) [arXiv:astro-ph/0307267]; for a review, see A. Kusenko, Int. J. Mod. Phys. D13, 2065 (2004) [arXiv:astro-ph/0409521].

[50] S. H. Hansen and Z. Haiman, Astrophys. J. 600, 26 (2004) [arXiv:astro-ph/0305126];

P. L. Biermann and A. Kusenko, Phys. Rev. Lett. 96, 091301 (2006) [arXiv:astro-ph/0601004].

[51] K. Abazajian, G. M. Fuller and W. H. Tucker, Astrophys. J. 562, 593 (2001) [arXiv:astro-ph/0106002].

[52] A. Boyarsky, A. Neronov, O. Ruchayskiy and M. Shaposhnikov, Phys. Rev. D 74, 103506 (2006) [arXiv:astro-ph/0603368]; A. Boyarsky, A. Neronov, O. Ruchayskiy, M. Shaposhnikov and I. Tkachev, Phys. Rev. Lett. 97, 261302 (2006) [arXiv:astro-ph/0603660].

[53] C. Boehm, Y. Farzan, T. Hambye, S. Palomares-Ruiz and S. Pascoli, arXiv: hep-ph/0612228.

[54] T. Asaka, S. Blanchet and M. Shaposhnikov, Phys. Lett. B 631, 151 (2005) [arXiv:hep-ph/0503065]; T. Asaka and M. Shaposhnikov, Phys. Lett. B 620, 17 (2005) [arXiv:hep-ph/0505013]; T. Asaka, M. Laine and M. Shaposhnikov, JHEP 0701, 091 (2007) [arXiv: hep-ph/0612182].

[55] T. Asaka, M. Shaposhnikov and A. Kusenko, Phys. Lett. B 638, 401 (2006) [arXiv:hep-ph/0602150].

[56] F. L. Bezrukov and M. Shaposhnikov, Phys. Rev. D 75, 053005 (2007) [arXiv:hep-ph/0611352]; D. Gorbunov and M. Shaposhnikov, JHEP 0710, 015 (2007) [arXiv:0705.1729 [hep-ph]].

[57] A. Y. Smirnov and R. Zukanovich Funchal, Phys. Rev. D 74, 013001 (2006) [arXiv:hep-ph/0603009].

[58] A. Kusenko, arXiv:hep-ph/0703116.

[59] G. Gelmini, E. Osoba, S. Palomares-Ruiz and S. Pascoli, JCAP 0810, 029 (2008) [arXiv:0803.2735 [astro-ph]].

[60] W. Chao, Z. G. Si, Z. Z. Xing and S. Zhou, Phys. Lett. B 666, 451 (2008) [arXiv:0804.1265[hep-ph]].

[61] F. E. Wietfeldt and E. B. Norman, Phys. Rept. 273, 149 (1996).

[62] B. Armbruster et al. [KARMEN Collaboration], Phys. Lett. B 348, 19 (1995).

[63] M. Daum et al., Phys. Rev. Lett. 85, 1815 (2000) [arXiv:hep-ex/0008014].

[64] M. Cirelli, G. Marandella, A. Strumia and F. Vissani, Nucl. Phys. B 708, 215 (2005) [arXiv:hep-ph/0403158].

[65] A. A. Aguilar-Arevalo et al. [The MiniBooNE Collaboration], arXiv:0704.1500 [hep-ex].

[66] M. Maltoni and T. Schwetz, arXiv:0705.0107 [hep-ph].

[67] R. E. Shrock, Phys. Lett. B 96, 159 (1980). 
[68] Z. Chacko, L. J. Hall, S. J. Oliver and M. Perelstein, Phys. Rev. Lett. 94, 111801 (2005) [arXiv: hep-ph/0405067]; S. Palomares-Ruiz, S. Pascoli and T. Schwetz, JHEP 0509, 048 (2005) [arXiv:hep-ph/0505216].

[69] D. Errede et al., Phys. Rev. D 37, 577 (1988); D. Decamp et al. [ALEPH Collaboration], Phys. Lett. B 236, 511 (1990); P. Burchat et al., Phys. Rev. D 41, 3542 (1990); B. Adeva et al. [L3 Collaboration], Phys. Lett. B 251, 321 (1990); P. Achard et al. [L3 Collaboration], Phys. Lett. B 517, 67 (2001) [arXiv:hep-ex/0107014].

[70] E. Nardi, E. Roulet and D. Tommasini, Phys. Lett. B 327, 319 (1994) [arXiv:hep-ph/9402224]; E. Nardi, E. Roulet, and D. Tommasini, Phys. Lett. B 344, 225 (1995) [arXiv:hep-ph/9409310].

[71] S. Bergmann and A. Kagan, Nucl. Phys. B 538, 368 (1999) [arXiv:hep-ph/9803305].

[72] F. del Aguila, J. de Blas and M. Perez-Victoria, Phys. Rev. D 78 (2008) 013010 [arXiv:0803.4008 [hep-ph]].

[73] E. Ma and A. Pramudita, Phys. Rev. D 24, 1410 (1981).

[74] P. Langacker and D. London, Phys. Rev. D 38, 907 (1988).

[75] D. Tommasini, G. Barenboim, J. Bernabeu and C. Jarlskog, Nucl. Phys. B 444, 451 (1995) [arXiv:hep-ph/9503228].

[76] M. Galeazzi, F. Fontanelli, F. Gatti and S. Vitale, Phys. Rev. Lett. 86, 1978 (2001).

[77] K. H. Hiddemann, H. Daniel and O. Schwentker, J. Phys. G 21, 639 (1995).

[78] E. Holzschuh, W. Kundig, L. Palermo, H. Stussi and P. Wenk, Phys. Lett. B 451, 247 (1999).

[79] E. Holzschuh, L. Palermo, H. Stussi and P. Wenk, Phys. Lett. B 482, 1 (2000).

[80] J. Deutsch, M. Lebrun and R. Prieels, Nucl. Phys. A 518, 149 (1990).

[81] H. O. Back et al., JETP Lett. 78, 261 (2003) [Pisma Zh. Eksp. Teor. Fiz. 78, 707 (2003)].

[82] C. Hagner, M. Altmann, F. von Feilitzsch, L. Oberauer, Y. Declais and E. Kajfasz, Phys. Rev. D 52, 1343 (1995).

[83] D. I. Britton et al., Phys. Rev. Lett. 68, 3000 (1992); D. I. Britton et al., Phys. Rev. D 46, 885 (1992).

[84] P. Benes, A. Faessler, F. Simkovic and S. Kovalenko, Phys. Rev. D 71, 077901 (2005) [arXiv:hep-ph/0501295].

[85] D. Berghofer et al., Proc. Intern. Conf. on Neutrino Physics and Astrophysics (Maui, Hawaii, 1981), 67 (1981), eds. R. J. Cence, E. Ma and A. Roberts, Vol. II (University of Hawaii, Honolulu, HI, 1981); T. Yamazaki, Proc. 22nd Intern. Conf, on High-energy physics (Leipzig, 1984), 262 (1984), eds. A. Meyer and E. Wieczorek, Vol. I (Akademie der Wiessenachaften der DDR, Leipzig, 1984).

[86] G. Bernardi et al., Phys. Lett. B 203, 332 (1988).

[87] J. Badier et al. [NA3 Collaboration], Z. Phys. C 31, 21 (1986).

[88] F. Bergsma et al. [CHARM Collaboration], Phys. Lett. B 166, 473 (1986). 
[89] P. Abreu et al. [DELPHI Collaboration], Z. Phys. C 74, 57 (1997) [Erratum-ibid. C 75, 580 (1997)].

[90] O. Adriani et al. [L3 Collaboration], Phys. Lett. B 295, 371 (1992).

[91] G. Bélanger, F. Boudjema, D. London, and H. Nadeau, Phys. Rev. D53, 6292 (1996) [arXiv:hep-ph/9508317]; D. London, arXiv:hep-ph/9907419.

[92] A. Kusenko, S. Pascoli and D. Semikoz, JHEP 0511, 028 (2005) [arXiv:hep-ph/0405198].

[93] A. M. Cooper-Sarkar et al., Phys. Lett. 160 B, 207 (1985).

[94] E. Gallas et al. [FMMF Collaboration], Phys. Rev. D 52, 6 (1995).

[95] A. Vaitaitis et al. [NuTeV Collaboration], Phys. Rev. Lett. 83, 4943 (1999) [arXiv:hep-ex/9908011].

[96] P. Vilain et al. [CHARM II Collaboration], Phys. Lett. B 343, 453 (1995) [Phys. Lett. B 351, 387 (1995)].

[97] J. Orloff, A. N. Rozanov and C. Santoni, Phys. Lett. B 550, 8 (2002) [arXiv:hep-ph/0208075].

[98] P. Astier et al. [NOMAD Collaboration], Phys. Lett. B 506, 27 (2001) [arXiv:hep-ex/0101041].

[99] PDG, S. Eidelman et al. Phys. Lett. B592, 1 (2004).

[100] F. del Aguila, private communication.

[101] M. L. Brooks et al. [MEGA Collaboration], Phys. Rev. Lett. 83, 1521 (1999) [arXiv:hep-ex/9905013].

[102] C. Dohmen et al. [SINDRUM II Collaboration.], Phys. Lett. B 317, 631 (1993).

[103] B. Aubert et al. [BaBar Collaboration], Phys. Rev. Lett. 95, 191801 (2005)[arXiv: hep-ex/0506066].

[104] Q. He et al. [CLEO Collaboration], Phys. Rev. Lett. 95, 221802 (2005)[arXiv: hep-ex/0508031].

[105] Y. Grossman, Z. Ligeti and E. Nardi, Phys. Rev. D55, 2768 (1997) [arXiv:hep-ph/9607473].

[106] A. Abulencia et al. [CDF Collaboration], Phys. Rev. Lett. 98, 221803 (2007) [arXiv:hep-ex/0702051].

[107] ATLAS Collaboration, Detector and Physics Performances Technical Design Report, Vol.I, ATLAS TDR 14, CERN/LHCC 99-14, Section 2.5.9.

[108] A. Abulencia et al. [CDF Collaboration], Phys. Rev. Lett. 96, 011802 (2006) [arXiv:hep-ex/0508051].

[109] A. Hektor, M. Kadastik, M. Muntel, M. Raidal and L. Rebane, Nucl. Phys. B 787, 198 (2007) [arXiv:0705.1495 [hep-ph]]; P. Fileviez Perez, T. Han, G.-Y. Huang, T. Li and K. Wang, arXiv:0803.3450 [hep-ph].

[110] P. Achard et al., Phys. Lett. B 517, 67 (2001) [arXiv:hep-ex/0107014]. 
[111] U. Seljak et al., Phys. Rev. D71, 103515 (2005) [arXiv:astro-ph/0407372].

[112] F. Maltoni and T. Stelzer, JHEP 0302:027 (2003) [arXiv:hep-ph/0208156].

[113] M. A. Ivanov and S. G. Kovalenko, Phys. Rev. D71, 053004 (2005) [arXiv:hep-ph/0412198].

[114] D. Ebert, R. N. Faustov and V. O. Galkin, Phys. Lett. B635, 93 (2006) [arXiv:hep-ph/0602110].

[115] M. Artuso et al. [CLEO Collaboration], Phys. Rev. Lett. 95, 251801 (2005) [arXiv:hep-ex/0508057].

[116] C. Bernard et al. [MILC Collaboration], Phys. Rev. D66, 094501 (2002) [arXiv:hep-lat/0206016].

[117] T. Feldmann, Int. J. Mod. Phys. A15, 159 (2000) [arXiv:hep-ph/9907491].

[118] K. W. Edwards et al. [CLEO Collaboration], Phys. Rev. Lett. 86, 30 (2001) [arXiv:hep-ex/0007012].

[119] G.-L. Wang, Phys. Lett. B633, 492 (2006) [arXiv:math-ph/0512009]. 